TRANSACTIONS OF THE

AMERICAN MATHEMATICAL SOCIETY

Volume 350, Number 5, May 1998, Pages 1713-1790

S 0002-9947(98)01763-2

\title{
BOUNDARY SLOPES OF PUNCTURED TORI IN 3-MANIFOLDS
}

\author{
C. McA. GORDON
}

\begin{abstract}
Let $M$ be an irreducible 3-manifold with a torus boundary component $T$, and suppose that $r, s$ are the boundary slopes on $T$ of essential punctured tori in $M$, with their boundaries on $T$. We show that the intersection number $\Delta(r, s)$ of $r$ and $s$ is at most 8. Moreover, apart from exactly four explicit manifolds $M$, which contain pairs of essential punctured tori realizing $\Delta(r, s)=8,8,7$ and 6 respectively, we have $\Delta(r, s) \leq 5$. It follows immediately that if $M$ is atoroidal, while the manifolds $M(r), M(s)$ obtained by $r$ and $s$-Dehn filling on $M$ are toroidal, then $\Delta(r, s) \leq 8$, and $\Delta(r, s) \leq 5$ unless $M$ is one of the four examples mentioned above.

Let $\mathcal{H}_{0}$ be the class of 3 -manifolds $M$ such that $M$ is irreducible, atoroidal, and not a Seifert fibre space. By considering spheres, disks and annuli in addition to tori, we prove the following. Suppose that $M \in \mathcal{H}_{0}$, where $\partial M$ has a torus component $T$, and $\partial M-T \neq \varnothing$. Let $r, s$ be slopes on $T$ such that $M(r), M(s) \notin \mathcal{H}_{0}$. Then $\Delta(r, s) \leq 5$. The exterior of the Whitehead sister link shows that this bound is best possible.
\end{abstract}

\section{INTRODUCTION}

Let $M$ be a 3-manifold and $T$ a torus component of $\partial M$. (Throughout, all 3manifolds will always be assumed to be compact, connected, and orientable.) With no real loss of generality, we shall assume that $M$ is irreducible. Recall that the slope of an essential unoriented simple closed curve on $T$ is its isotopy class, and that if $r$ and $s$ are two slopes on $T$ then $\Delta(r, s)$ denotes their minimal geometric intersection number.

Let $(F, \partial F) \subset(M, \partial M)$ be an essential surface with $\partial F \cap T \neq \emptyset$. Then all the components of $\partial F \cap T$ have the same slope on $T$, the boundary slope of $F$ on $T$. Consideration of such surfaces naturally arises in the context of Dehn filling. More precisely, if $r$ is a slope on $T$, let $M(r)$ be the manifold obtained from $M$ by $r$-Dehn filling, that is, by attaching a solid torus $V$ to $M$ along $T$ so that $r$ bounds a disk in $V$. If $M(r)$ contains an essential surface $S$, then either $S$ can be moved off $V$ into $M$, or there is an essential surface $F$ in $M$ with boundary slope $r$ on $T$ such that $\widehat{F}$, the surface obtained from $F$ by capping off the components of $\partial F \cap T$ with disks, is homeomorphic to $S$. In this paper we shall be mainly concerned with the case where $\widehat{F}$ is a torus; let $\mathcal{T}(M, T)$ denote the set of boundary slopes on $T$ of such punctured tori $F$ in $M$.

Interesting examples are provided by some of the manifolds obtained by Dehn surgery on one component of the Whitehead link. Let $W$ be the exterior of the Whitehead link and let $T_{0}$ be a boundary component of $W$. Choosing a standard

Received by the editors March 27, 1995 and, in revised form, February 26, 1996.

1991 Mathematics Subject Classification. Primary 57M25; Secondary 57M50.

(C)1998 American Mathematical Society 
meridian-longitude basis $\mu, \lambda$ for $H_{1}\left(T_{0}\right)$, we may parametrize the slopes on $T_{0}$ by $\mathbb{Q} \cup\{1 / 0\}$ in the usual way, by associating $r$ with $p / q$ if $[r]=p \mu+q \lambda \in H_{1}\left(T_{0}\right)$, and write $W(r)=W(p / q)$.

Then [Ho] for $M=W(2), W(-5 / 2), W(-5)$, and $W(1)$, int $M$ is hyperbolic (so $M$ is irreducible and contains no essential torus), whilst there exist slopes $r, s$ on $\partial M$ with $\Delta(r, s)=6,7,8$, and 8 respectively such that $M(r)$ and $M(s)$ each contains an essential torus. Hence $r, s \in \mathcal{T}(M, \partial M)$. Our main result asserts that these examples are extremal.

Theorem 1.1. Let $M$ be an irreducible 3-manifold and $T$ a torus component of $\partial M$. If $r, s \in \mathcal{T}(M, T)$ then either

(1) $\Delta(r, s) \leq 5$; or

(2) $\Delta(r, s)=6$ and $M$ is homeomorphic to $W(2)$; or

(3) $\Delta(r, s)=7$ and $M$ is homeomorphic to $W(-5 / 2)$; or

(4) $\Delta(r, s)=8$ and $M$ is homeomorphic to either $W(1)$ or $W(-5)$.

We remark that $W(1)$ is homeomorphic to the exterior of the figure eight knot, and $\operatorname{vol} W(1)=\operatorname{vol} W(-5), \operatorname{vol} W(-5 / 2), \operatorname{vol} W(2)$ are respectively the lowest, second lowest, and third lowest known volumes of hyperbolic 3-manifolds with a single cusp. Also, in each case there are exactly two slopes $r, s$ with the stated property.

Theorem 1.1 has an obvious corollary about the creation of essential tori by Dehn filling. It is natural to consider also how other surfaces of non-negative euler characteristic might arise, that is, the cases where $\widehat{F}$ is a sphere, disk or annulus. For instance, let us consider tori and spheres. As an example here, let $M$ be the exterior of the trefoil knot. Then (parametrizing slopes on $\partial M$ by meridianlongitude coordinates) $M(0)$ is a torus bundle over the circle, and $M(6)$ is the connected sum of the lens spaces $L(2,1)$ and $L(3,1)$. We then have the following result. Let $\mathcal{A}$ denote the class of irreducible 3 -manifolds that contain no essential tori.

Theorem 1.2. Suppose that $M \in \mathcal{A}$ and that $\partial M$ has a torus component $T$. Let $r, s$ be slopes on $T$ such that $M(r), M(s) \notin \mathcal{A}$. Then either one of conclusions (1)(4) of Theorem 1.1 holds, or $\Delta(r, s)=6$ and $M$ is homeomorphic to the exterior of the trefoil knot.

Using Thurston's uniformization theorem for Haken manifolds [T], we obtain a bound on the degeneration of hyperbolic structures under Dehn filling, at least in the case where the resulting manifold has non-empty boundary. To best state the result, let $\mathcal{H}$ denote the class of 3-manifolds $M$ such that int $M$ has a complete hyperbolic structure, and let $\mathcal{H}_{0}$ be the set of elements of $\mathcal{H}$ that are not Seifert fibre spaces. Thus $\mathcal{H}_{0}=\mathcal{H}-\left\{S^{1} \times D^{2}, T^{2} \times I\right\}$. Thurston has shown [T] that if $M$ is a 3-manifold with non-empty boundary, then $M \in \mathcal{H}_{0}$ if and only if $M \in \mathcal{A}$ and is not a Seifert fibre space. By considering annuli and disks in addition to spheres and tori, we obtain the following theorem.

Theorem 1.3. Suppose that $M \in \mathcal{H}_{0}$, where $\partial M$ has a torus component $T$ and at least one other component. Let $r, s$ be slopes on $T$ such that $M(r), M(s) \notin \mathcal{H}_{0}$. Then $\Delta(r, s) \leq 5$.

Theorem 1.3 applies in particular to hyperbolic links in $S^{3}$ with at least two components. In fact, there is an example of a two-component link in $S^{3}$ which 
shows that the bound of 5 in Theorem 1.3 (and Theorems 1.1 and 1.2) cannot be improved. This is the Whitehead sister link illustrated in [BFLW, Figure 3]; one component is unknotted and the other is a trefoil. Its exterior $M$ has a hyperbolic structure with the same volume as the exterior of the Whitehead link. For each of the two boundary components $T$ of $M$, there are slopes $r, s$ on $T$ with $\Delta(r, s)=5$ such that $M(r)$ and $M(s)$ contain essential tori [Ho].

In the case of Dehn filling on a hyperbolic manifold $M$ with a single boundary component, one conjectures of course that if $M \in \mathcal{H}_{0}$ and $M(r), M(s) \notin \mathcal{H}_{0}$, then $\Delta(r, s) \leq 8$. In general, our topological methods give no information on this beyond Theorem 1.2. (Recall that it is a special case of Thurston's geometrization conjecture that if $M \in \mathcal{A}$ and $\pi_{1}(M)$ is infinite, then either $M \in \mathcal{H}$ or $M$ belongs to a certain small class of Seifert fibre spaces.) Using Thurston's uniformization theorem for closed Haken manifolds, however, we get a result in the following special case.

Theorem 1.4. Suppose that $M \in \mathcal{H}$ and that $\partial M$ is a torus. Suppose that int $M$ contains a closed incompressible surface $S$ such that there is no incompressible annulus in $M$ joining $S$ to $\partial M$. Let $r, s$ be slopes on $\partial M$ such that $M(r), M(s) \notin \mathcal{H}$. Then $\Delta(r, s) \leq 5$.

We now give a brief description of the organization of the paper.

As in [GLi], which considers boundary slopes of punctured spheres, a special role in our arguments is played by those pairs $(M, T)$ that are cabled (see Section 2 for the definition). More specifically, we prove Theorem 1.1 in two parts, in the following propositions.

Proposition 1.5. If $(M, T)$ is not cabled and $r, s \in \mathcal{T}(M, T)$, then the conclusion of Theorem 1.1 holds.

Proposition 1.6. If $(M, T)$ is cabled and $r, s \in \mathcal{T}(M, T)$, then $\Delta(r, s) \leq 4$.

We remark that the bound of 4 in Proposition 1.6 is also sharp (see Section 13).

The main part, Proposition 1.5, is proved by analysing in detail the possible patterns of intersection of two punctured tori in $M$.

In Section 2 we prove some general lemmas about intersections of essential surfaces, whilst Section 3 contains the results we need about graphs in surfaces. The "generic" case of Proposition 1.5 is done in Section 4; the argument here is based on [GLi]. The completion of the proof of Proposition 1.5 occupies Sections 5-11. After some preparatory lemmas in Section 5, Sections 6-10 treat the various cases that remain after the results of Section 4 . The conclusion is that if $(M, T)$ is not cabled and $\left(F_{\alpha}, \partial F_{\alpha}\right) \subset(M, T)$ is an essential punctured torus with boundary slope $r_{\alpha}, \alpha=1,2$, such that $\Delta=\Delta\left(r_{1}, r_{2}\right) \geq 6$, then (after isotoping the surfaces so as to minimize their intersection) the only possibilities for $\left(F_{1}, F_{2} ; F_{1} \cap F_{2}\right)$ are: one with $\Delta=6$, an infinite family with $\Delta=6$, one with $\Delta=7$, and two with $\Delta=8$. In Section 11 we show that for the infinite family with $\Delta=6$, one of the surfaces $F_{\alpha}$ must actually be inessential, whilst in each of the other four cases the triple $\left(M ; F_{1}, F_{2}\right)$ is uniquely determined. These must therefore correspond to the four examples listed in Theorem 1.1.

In Section 12 we consider other pairs of essential surfaces $F_{\alpha}$ in $M$ such that $\widehat{F}_{\alpha}$ has non-negative euler characteristic, $\alpha=1,2$, and show, modulo cabling (or, in some cases, modulo the existence of essential annuli in $M$ ), that $\Delta\left(r_{1}, r_{2}\right) \leq 5$. In general this bound is not best possible, and in some cases stronger results are 
known, but as it is sufficient for Theorems 1.2, 1.3, and 1.4 we do not discuss the matter further. (For some recent results on these questions see [Wu1], [GLu], [BZ1], [BZ2], $[\mathrm{H}]$ and $[\mathrm{HM}]$.

In Section 13 we consider the case where $(M, T)$ is cabled, proving Proposition 1.6 and analogs for other surfaces of non-negative euler characteristic. The argument here makes use of the classification of essential planar surfaces in cable spaces given in [GLi]. We remark that for many applications, for example to Theorems 1.2, 1.3, and 1.4, consideration of cabled manifolds is not really necessary, as such a manifold either contains an essential torus or is a very special Seifert fibre space. However, expressed in terms of boundary slopes, the ultimate results apply (in most cases) equally well to cabled manifolds, so we carry out an analysis of the cabled case that is sufficient to give, for tori, Theorem 1.1, and for other surfaces of non-negative euler characteristic, the results stated in Section 14, Theorems 14.2, 14.3 and 14.5.

Finally, also in Section 14, we prove Theorems 1.2, 1.3, and 1.4.

I should like to thank Craig Hodgson for his help in telling me about the examples $W(-5), W(-5 / 2), W(2)$, and the Whitehead sister. The information given above about them and their toral boundary slopes was obtained by him using Jeffrey Weeks' Dehn surgery computer program "snappea." The manifold $W(-5)$ is also discussed at length in Weeks' thesis [We].

\section{IntERSECTIONS OF ESSENTIAL SURFACES}

By a surface we shall mean a compact, connected, orientable 2-manifold, and we shall say that a surface in a 3-manifold is essential if it is properly embedded and either (i) incompressible, not parallel to a subsurface of the boundary of the 3 -manifold, and not a 2-sphere, or (ii) a 2-sphere that does not bound a 3-ball.

Now let $M$ be an irreducible 3-manifold (one that does not contain an essential 2 -sphere), and let $T$ be a torus component of $\partial M$. We assume that $T$ is incompressible. (If not, $M$ is a solid torus.) Let $F_{\alpha}, \alpha=1,2$, be an essential surface in $M$ such that $\partial F_{\alpha} \cap T$ has $n_{\alpha}>0$ components. These all have slope $r_{\alpha}$, say, on $T$. Let $\Delta=\Delta\left(r_{1}, r_{2}\right)$. By standard arguments, we may isotope $F_{1}$, say, so that $F_{1}$ and $F_{2}$ intersect transversely in a finite disjoint union of circles and properly embedded arcs, where each circle is essential in $F_{\alpha}$ and no arc with both its endpoints on $T$ is parallel in $F_{\alpha}$ to a subarc of $\partial F_{\alpha}, \alpha=1,2$, and each component of $\partial F_{1} \cap T$ meets each component of $\partial F_{2} \cap T$ in $\Delta$ points.

If $A$ is an arc component of $F_{1} \cap F_{2}$ with some endpoint on $T$, then, considering $A$ as it lies in $F_{\alpha}$, we label that endpoint with the (number of the) corresponding component of $\partial F_{\beta} \cap T$. (Here and throughout the paper we use the convention of $[\mathrm{L}]$ that $\{\alpha, \beta\}=\{1,2\}$.) Thus around each component of $\partial F_{\alpha} \cap T$ we see the labels $1,2, \ldots, n_{\beta}$ in cyclic order, this sequence being repeated $\Delta$ times. If we orient $F_{\alpha}$, then this induces an orientation on each component $a$ of $\partial F_{\alpha} \cap T$, and we assign a sign \pm to $a$ according to the direction of this orientation as $a$ lies on $T$. We adopt the convention that if the sign of $a$ is + , then the labels $1,2, \ldots, n_{\beta}$ appear in anticlockwise order around $a$, and if the sign is -, then they appear in clockwise order. This kind of labeling was first used in [L].

An arc component of $F_{1} \cap F_{2}$ with both endpoints on $T$ will be called an internal arc of $F_{1} \cap F_{2}$. The orientability of $M$ then gives us the parity rule: if $A$ is an internal arc of $F_{1} \cap F_{2}$ on $F_{\alpha}$, then the components of $\partial F_{\alpha} \cap T$ joined by $A$ have 

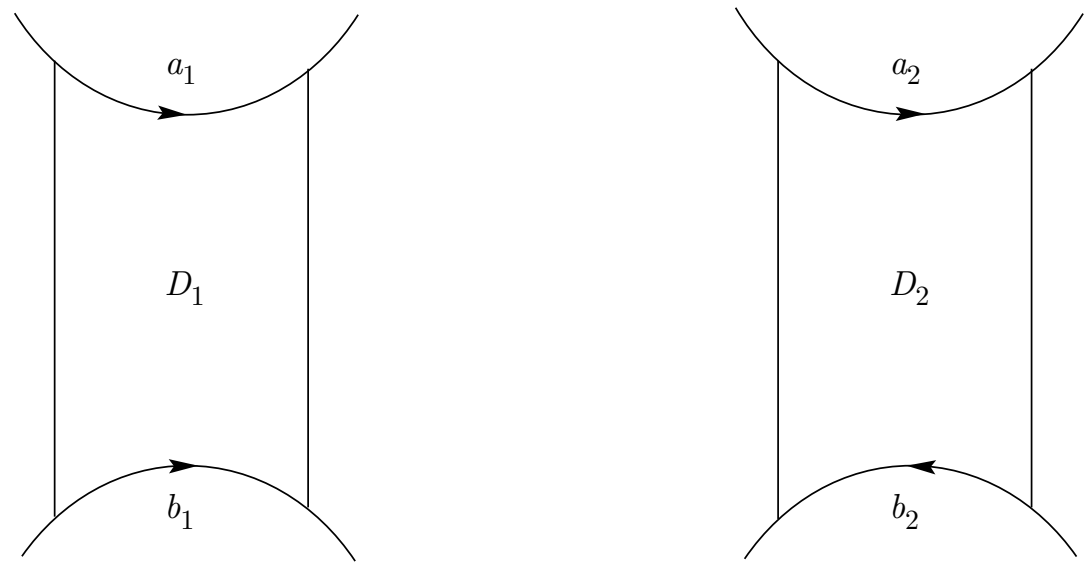

FIGURE 1

the same (resp. opposite) sign if and only if the labels at the endpoints of $A$ have opposite (resp. the same) sign.

Let $K_{p, q} \subset \operatorname{int} S^{1} \times D^{2}$ be a $(p, q)$-curve, $q \geq 2$, that is, a simple closed curve on the boundary of a concentric solid torus that winds around $p$ times meridionally and $q$ times longitudinally. The exterior of $K_{p, q}, S^{1} \times D^{2}-\operatorname{int} N\left(K_{p, q}\right)$, is called a $(p, q)$-cable space, and a pair $(M, T)$ is said to be cabled if $M$ contains a $(p, q)$-cable space $C$ with $C \cap \partial M=T$. It is easy to see that if $p \equiv \pm p^{\prime}(\bmod q)$ then the corresponding cable spaces are homeomorphic.

Lemma 2.1. Suppose that $F_{1} \cap F_{2}$ contains a pair of internal arcs that are parallel in both $F_{1}$ and $F_{2}$. Then $(M, T)$ is $(1,2)$-cabled.

Proof. Let $D_{1}$ and $D_{2}$ be disks (rectangles) that realize the parallelism of $\operatorname{arcs} A$ and $A^{\prime}$, in $F_{1}$ and $F_{2}$ respectively. By taking innermost such disks we may suppose that $D_{1} \cap D_{2}=A \cup A^{\prime}$. Let the arcs join components $a_{1}, b_{1}$ of $\partial F_{1}$ and $a_{2}, b_{2}$ of $\partial F_{2}$. Then $a_{1}$ and $b_{1}$ (say) are of opposite sign, whilst $a_{2}$ and $b_{2}$ are of the same sign. (See Figure 1, where the arrows indicate coherent directions on T.) A priori there are two possibilities for the way in which $D_{1}$ and $D_{2}$ are identified along $A$ and $A^{\prime}$, illustrated by the arrows on $A$ and $A^{\prime}$ in Figures 2(a) and (b).

In fact, case (a) is impossible. To see this, let $P, Q, R, S$ be the points, and $\alpha_{1}, \beta_{1}, \alpha_{2}, \beta_{2}$ the arcs, indicated in Figure 2(a). Note that $P, Q \in a_{1} \cap a_{2}$, and $R, S \in b_{1} \cap b_{2}$. Then

$$
\begin{aligned}
& \left|\alpha_{1} \cap b_{2}\right|=\left|\beta_{1} \cap a_{2}\right|=k_{1} \geq 1, \text { and } \\
& \left|\alpha_{2} \cap b_{1}\right|=\left|\beta_{2} \cap a_{1}\right|=k_{2} \geq 1 .
\end{aligned}
$$

Now consider the disjoint simple closed curves $\alpha=\alpha_{1} \cup \alpha_{2}$ and $\beta=\beta_{1} \cup \beta_{2}$ on $T$. Orient each pair $a_{1}, b_{1}$ and $a_{2}, b_{2}$ coherently on $T$ as in Figure 1 ; this orients $\alpha_{1}, \alpha_{2}, \beta_{1}, \beta_{2}$. Now give $\alpha$ the orientation induced by $\alpha_{1}$ (which is equal to that induced by $-\alpha_{2}$ ), and $\beta$ the orientation induced by $\beta_{1}$ (which is equal to that induced by $\beta_{2}$ ). Then (with some convention) we have the following algebraic intersection numbers:

$$
\begin{aligned}
& \alpha \cdot a_{1}=\alpha \cdot b_{1}=\left(-\alpha_{2}\right) \cdot b_{1}=-k_{2}, \\
& \beta \cdot a_{1}=\beta_{2} \cdot a_{1}=k_{2} .
\end{aligned}
$$



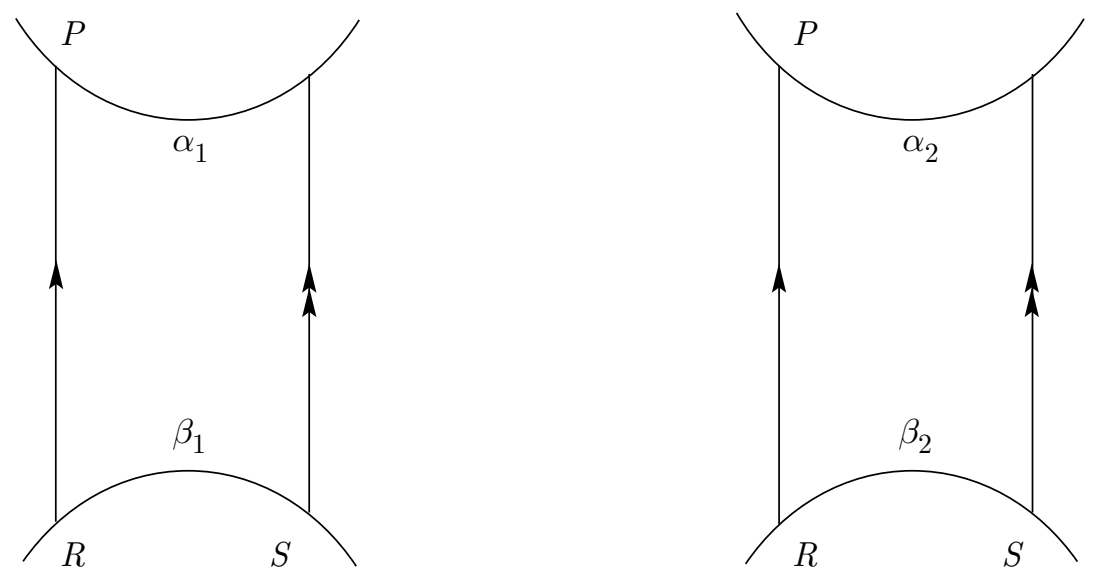

(a)
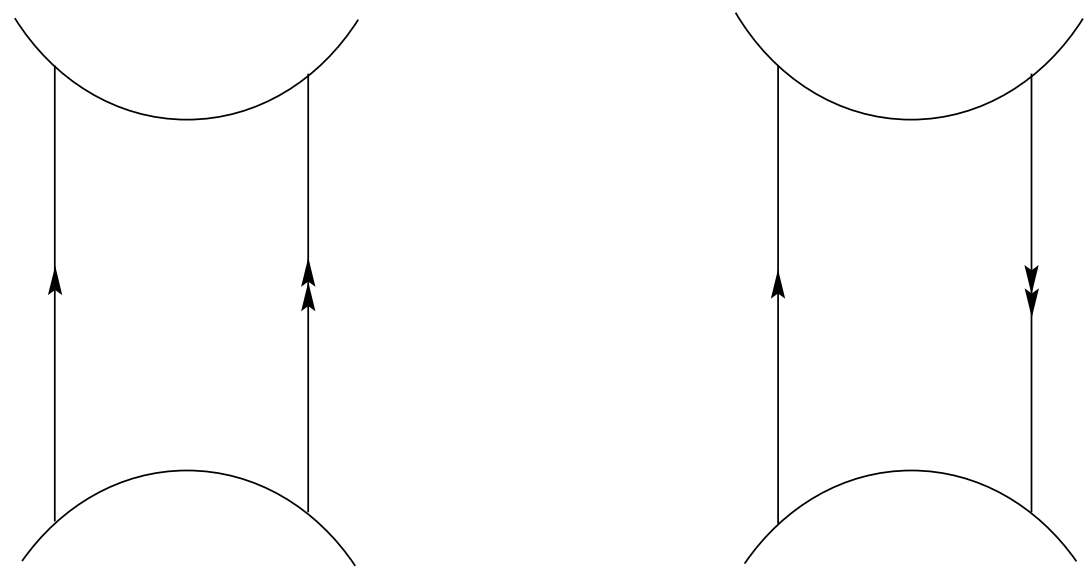

(b)

Figure 2

On the other hand, if $a_{2} \neq b_{2}$, then

$$
\begin{aligned}
& \alpha \cdot a_{2}=\alpha \cdot b_{2}=\alpha_{1} \cdot b_{2}=k_{1}, \\
& \beta \cdot a_{2}=\beta_{1} \cdot a_{2}=k_{1} .
\end{aligned}
$$

This contradicts the fact that $\alpha$ and $\beta$ are disjoint.

Similarly, if $a_{2}=b_{2}$, then $k_{1} \geq 2$, and we have

$$
\begin{aligned}
& \alpha \cdot a_{2}=k_{1}-1, \\
& \beta \cdot a_{2}=k_{1}-1 .
\end{aligned}
$$

This is again a contradiction.

In case (b), $D_{1} \cup D_{2}$ is a Möbius band $B$ with $(B, \partial B) \subset(M, T)$, from which it easily follows that $(M, T)$ is $(1,2)$-cabled. (See [GLi, Proposition 1.3, case (1)].) 


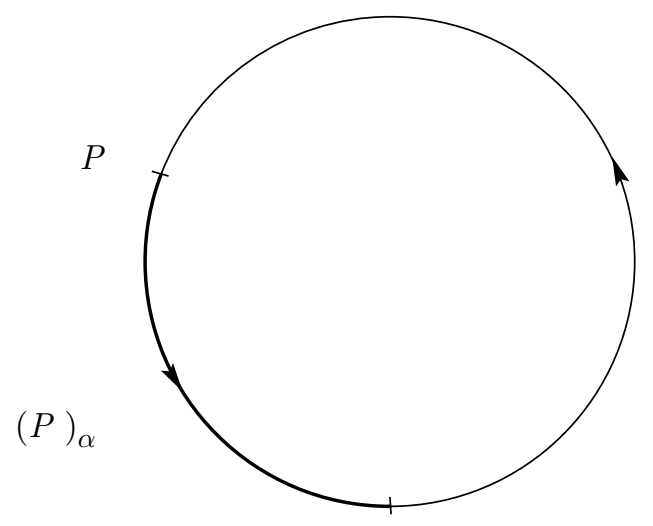

FiguRE 3

Suppose that $F_{\alpha}$ contains a family $\boldsymbol{A}$ of $n_{\beta}$ mutually parallel internal arcs of $F_{1} \cap F_{2}$, joining boundary components $a$ and $b$ (possibly equal) of $F_{\alpha}$. Let $A_{i}$ be the arc with label $i$ at one end of $\boldsymbol{A}, i=1,2, \ldots, n_{\beta}$. Then $A_{i}$ has label $\pi(i)$ at the other end, for some permutation $\pi$ of $\left\{1,2, \ldots, n_{\beta}\right\}$. Observe that $\pi$ is of the form

$$
\pi(i) \equiv \varepsilon i+p \quad\left(\bmod n_{\beta}\right)
$$

where $\varepsilon= \pm 1$ according as $a$ and $b$ are of opposite sign or of the same sign. The family $\boldsymbol{A}$ determines $\pi$ up to inversion.

If $\varepsilon=+1$, then any orbit of $\pi$ contains exactly $n_{\beta} /\left(n_{\beta}, p\right)$ elements, all of the same sign. If $\varepsilon=-1$, then any orbit of $\pi$ contains exactly 2 elements, of opposite sign (hence $n_{\beta}$ is even). In particular, the following lemma is immediate.

Lemma 2.2. Suppose that $F_{\alpha}$ contains $n_{\beta}$ mutually parallel internal arcs of $F_{1} \cap F_{2}$ such that the corresponding permutation has only one orbit. Then either

(1) $\partial F_{\beta}$ has exactly two components, of opposite sign; or

(2) all components of $\partial F_{\beta}$ are of the same sign.

Let $\theta$ be an orbit of $\pi$. The $\operatorname{arcs} A_{i}, i \in \theta$, define a circle $C_{\theta}(\boldsymbol{A})$ in $\widehat{F}_{\beta}$ in the obvious way. The following lemma is essentially proved in [GLi, Section 5].

Lemma 2.3. Suppose that $F_{\alpha}$ contains a family $\boldsymbol{A}$ of $n_{\beta}$ mutually parallel internal arcs of $F_{1} \cap F_{2}$, such that for some orbit $\theta$ of the corresponding permutation, $C_{\theta}(\boldsymbol{A})$ bounds a disk in $\widehat{F}_{\beta}$. Then $(M, T)$ is cabled.

Our next goal is to prove Lemma 2.5, which gives necessary conditions for a pair of internal arcs in $F_{\beta}$ to be parallel in $F_{\alpha}$. This will be used repeatedly in Sections 6 through 9 to rule out certain configurations of arcs in $F_{1} \cap F_{2}$.

Orient all the components of $\partial F_{\alpha} \cap T$ coherently on $T, \alpha=1,2$. If $P$ and $Q$ are two (distinct) points in some component $a$ of $\partial F_{\alpha} \cap T$, let $(P Q)_{\alpha}$ denote the arc in $a$ that goes from $P$ to $Q$ with respect to our chosen orientation of $a$. (See Figure 3.) 


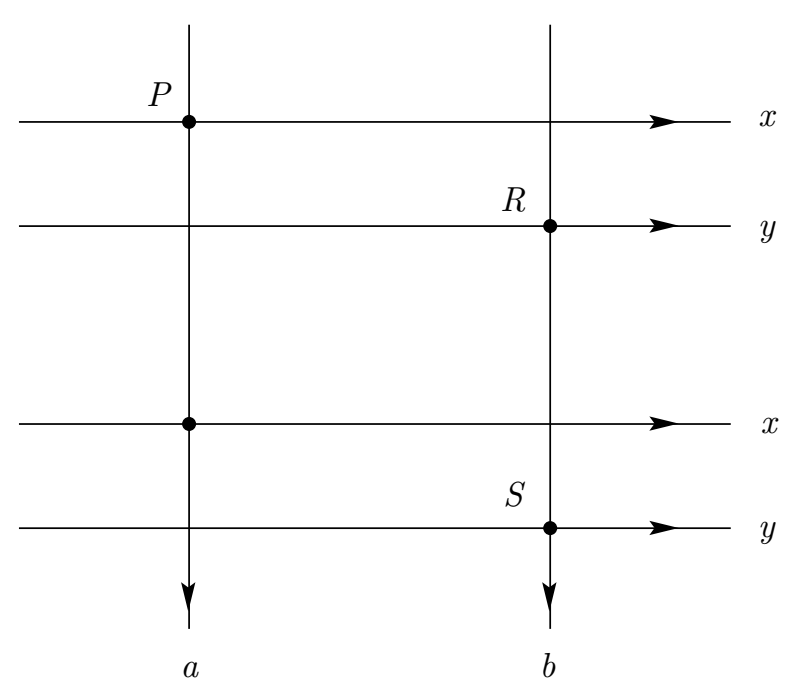

FiguRE 4

Suppose now that $P, Q \in a \cap \partial F_{\beta}$. Then we define

$$
\tau_{\alpha}(P, Q)=\left|(P Q)_{\alpha} \cap \partial F_{\beta}\right|-1 .
$$

In other words, $\tau_{\alpha}(P, Q)$ is (the number of labels on the $\left.\operatorname{arc}(P Q)_{\alpha}\right)-1$. Note that reversing the orientation of $a$ sends $\tau_{\alpha}(P, Q)$ to $\tau_{\alpha}(Q, P)=\Delta n_{\beta}-\tau_{\alpha}(P, Q)$.

Lemma 2.4. Let $a, b$ be components of $\partial F_{\alpha} \cap T$ and $x, y$ components of $\partial F_{\beta} \cap T$.

(i) Suppose that $P, Q \in a \cap x$ and $R, S \in b \cap y$. If $\tau_{\alpha}(P, Q)=\tau_{\alpha}(R, S)$ then $\tau_{\beta}(P, Q)=\tau_{\beta}(R, S)$.

(ii) Suppose that $P \in a \cap x, Q \in a \cap y, R \in b \cap x$, and $S \in b \cap y$. If $\tau_{\alpha}(P, Q)=\tau_{\alpha}(R, S)$ then $\tau_{\beta}(P, R)=\tau_{\beta}(Q, S)$.

Proof. (i) The situation is shown in Figure 4. (Note that we allow the possibilities $a=b, x=y$. $)$ Since $\tau_{\alpha}(P, Q)=\tau_{\alpha}(R, S)$, there is a homeomorphism of $\left(T ; \partial F_{1} \cap T\right.$, $\left.\partial F_{2} \cap T\right)$ taking $P$ to $R$ and $Q$ to $S$. Hence $\tau_{\beta}(P, Q)=\tau_{\beta}(R, S)$.

(ii) This situation is shown in Figure 5. Again, since $\tau_{\beta}(P, Q)=\tau_{\alpha}(R, S)$ there is a homeomorphism of $\left(T ; \partial F_{1} \cap T, \partial F_{2} \cap T\right)$ taking $P$ to $R$ and $Q$ to $S$. Hence $\tau_{\beta}(P, R)=\tau_{\beta}(Q, S)$.

We apply Lemma 2.4 to the situation where $P, Q, R$ and $S$ are the endpoints of two parallel arcs in $F_{\alpha}$. It is sometimes convenient to express the result in terms of the following variant of $\tau_{\alpha}$. Namely, instead of orienting the components of $\partial F_{\alpha} \cap T$ coherently on $T$, give them the orientation induced by some orientation of $F_{\alpha}$, and then define $\delta_{\alpha}$ in exactly the same way as $\tau_{\alpha}$. Thus $\delta_{\alpha}(P, Q)=\tau_{\alpha}(P, Q)$ or $\tau_{\alpha}(Q, P)$, depending on the sign of the component $a$ of $\partial F_{\alpha} \cap T$ containing $P$ and $Q$.

Lemma 2.5. Let $A$ and $B$ be internal arcs of $F_{1} \cap F_{2}$ that are parallel in $F_{\alpha}$, with endpoints $P, R$ and $Q, S$ respectively, labeled so that the boundary of the rectangle in $F_{\alpha}$ that realizes the parallelism is PQSR (see Figure 6). Let $x, y$ be components of $\partial F_{\beta} \cap T$. 


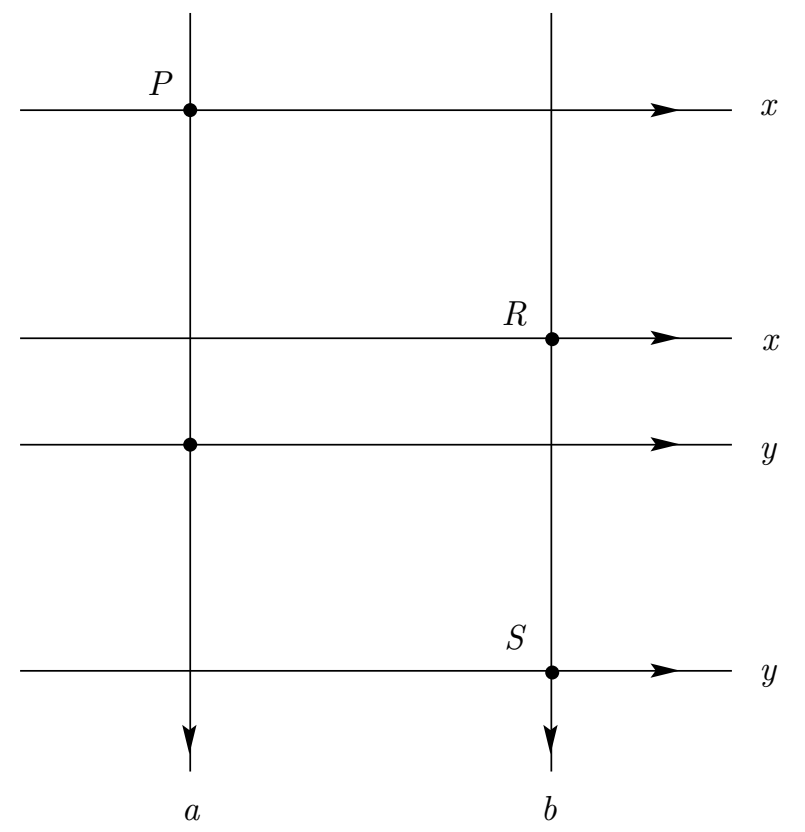

FiguRE 5

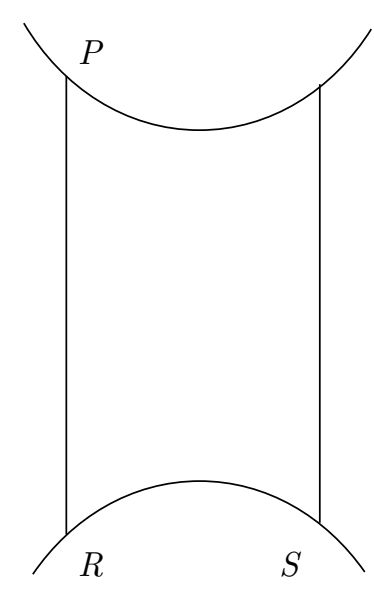

Figure 6

(i) Suppose that $P, Q \in x$ and $R, S \in y$ (see Figure $7(i)$ ). Then $\delta_{\beta}(P, Q)=$ $\delta_{\beta}(R, S)$.

(ii) Suppose that $P, S \in x, Q, R \in y$ (see Figure $7(i i)$ ), and that $x$ and $y$ are of opposite sign. Then $\delta_{\beta}(P, S)=\delta_{\beta}(R, Q)$.

(iii) Suppose that $P, R \in x$ and $Q, S \in y$ (see Figure 7(iii)). Then $\tau_{\beta}(P, R)=$ $\tau_{\beta}(Q, S)$.

Proof. Let $a$ and $b$ be the components of $\partial F_{\alpha} \cap T$ containing $P$ and $R$ respectively. 


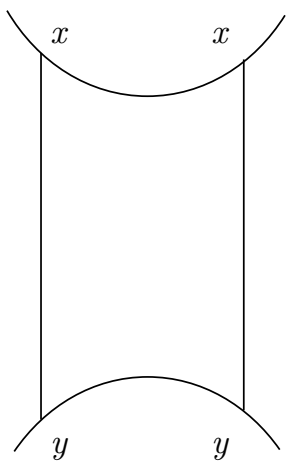

(i)

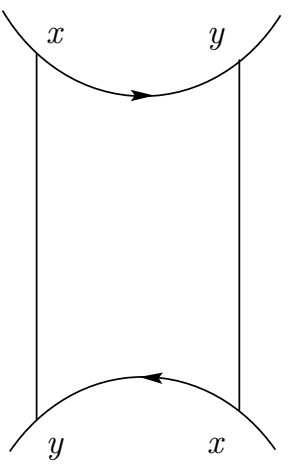

(ii)

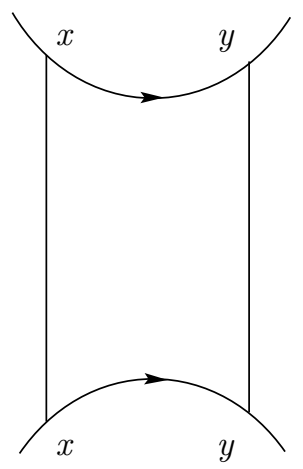

(iii)

FiguRE 7

(i) First suppose that $a$ and $b$ are of the same sign. Then $\tau_{\alpha}(P, Q)=\tau_{\alpha}(S, R)$. Therefore, by Lemma $2.4(\mathrm{i}), \tau_{\beta}(P, Q)=\tau_{\beta}(S, R)$. Since $x$ and $y$ are of opposite sign, this implies that $\delta_{\beta}(P, Q)=\delta_{\beta}(R, S)$.

If $a$ and $b$ are of opposite sign, then $\tau_{\alpha}(P, Q)=\tau_{\alpha}(R, S)$, and hence $\tau_{\beta}(P, Q)=$ $\tau_{\beta}(R, S)$. Since $x$ and $y$ are now of the same sign, we get $\delta_{\beta}(P, Q)=\delta_{\beta}(R, S)$ as before.

(ii) Since $x$ and $y$ are of opposite sign, $a$ and $b$ are of the same sign. Therefore $\tau_{\alpha}(P, Q)=\tau_{\alpha}(S, R)$, and hence, by Lemma 2.4(ii), $\tau_{\beta}(P, S)=\tau_{\beta}(Q, R)$, giving $\delta_{\beta}(P, S)=\delta_{\beta}(R, Q)$.

(iii) Here, since both endpoints of $A$ lie on the same component of $\partial F_{\beta} \cap T$ (and similarly for $B), a$ and $b$ are necessarily of opposite sign. Therefore $\tau_{\alpha}(P, Q)=$ $\tau_{\alpha}(R, S)$. By Lemma 2.4(ii), this implies that $\tau_{\beta}(P, R)=\tau_{\beta}(Q, S)$.

In practice, it is convenient to express Lemma 2.5 as follows. Let $A$ be an internal arc of $F_{1} \cap F_{2}$ in $F_{\beta}$, whose endpoints have label $a$ at boundary component $x$ and label $b$ at boundary component $y$. If $x \neq y$, then we can use $A^{x}$ to unambiguously denote the endpoint of $A$ at $x$. Similarly, if $a \neq b$, then we can use $A(a)$ to unambiguously denote the endpoint of $A$ with label $a$. (Note that we can't have both $x=y$ and $a=b$, by the parity rule.)

Lemma 2.5 asserts that if $A$ and $B$ are internal arcs of $F_{1} \cap F_{2}$ in $F_{\beta}$ that are parallel in $F_{\alpha}$ then:

in case (ii), and case (i) when $x \neq y, \delta_{\beta}\left(A^{x}, B^{x}\right)=\delta_{\beta}\left(A^{y}, B^{y}\right)$;

in case (i) when $a \neq b, \delta_{\beta}(A(a), B(a))=\delta_{\beta}(A(b), B(b))$;

in case (iii) (where necessarily $a \neq b), \tau_{\beta}(A(a), A(b))=\tau_{\beta}(B(a), B(b)$ ).

We conclude this section with an observation about the points of intersection of $\partial F_{1}$ and $\partial F_{2}$ on $T$ which will be useful in the sequel.

Let $a_{\alpha}$ be a component of $\partial F_{\alpha} \cap T, \alpha=1,2$. Parametrize $a_{1}$ as $\mathbb{R} / \mathbb{Z}$ in such a way that the points of $a_{1} \cap a_{2}$ are $\{i / \Delta: i=1,2, \ldots, \Delta\}$. Then $a_{2}$ may be parametrized as $\mathbb{R} / \mathbb{Z}$ so that the points of $a_{1} \cap a_{2}$ have $\left(a_{1}, a_{2}\right)$-coordinates $\{(i / \Delta, d i / \Delta): i=$ $1,2, \ldots, \Delta\}$ for some integer $d=d_{12}$ coprime to $\Delta$. By re-orienting $a_{2}$ if necessary we may assume that $1 \leq d \leq \Delta / 2$. Note that $d_{21} \equiv \pm d_{12}^{-1}(\bmod \Delta)$. 


\section{GRAPHS IN SURFACES}

Given surfaces $F_{1}, F_{2}$ in $M$ as described in Section 2, it is convenient to regard the arcs of $F_{1} \cap F_{2}$ as defining a graph $\Gamma_{\alpha}$ in $\widehat{F}_{\alpha}, \alpha=1,2$. Thus we regard the disks $C \ell\left(\widehat{F}_{\alpha}-F_{\alpha}\right)$ as the "fat" vertices of $\Gamma_{\alpha}$, and the $\operatorname{arcs}$ of $F_{1} \cap F_{2}$ with at least one endpoint on $T$ as the edges of $\Gamma_{\alpha}$. We call an edge of $\Gamma_{\alpha}$ corresponding to an internal arc an internal edge, and an edge corresponding to an arc with exactly one endpoint on $T$ (and the other on $\partial \widehat{F}_{\alpha}$ ) a boundary edge. Since no internal arc of $F_{1} \cap F_{2}$ is boundary-parallel in $F_{\alpha}$, no face of $\Gamma_{\alpha}$ is a disk with one side.

In the rest of the paper we shall use the topological and graph-theoretic terminologies interchangeably, for instance sometimes referring to arcs of $F_{1} \cap F_{2}$ in $F_{\alpha}$ and boundary components of $F_{\alpha}$, and sometimes to edges and vertices of $\Gamma_{\alpha}$.

The next two lemmas are about graphs in tori. The first is a special case of [GLi, Lemma 6.2].

Lemma 3.1. Let $\Gamma$ be a graph in a torus such that no face of $\Gamma$ is a disk with one side. Suppose that, for some positive integer $n$, the valency of each vertex of $\Gamma$ is greater than $6 n$. Then $\Gamma$ has $n+1$ mutually parallel edges.

If $\Gamma$ is a graph in a surface $F$ as described above, then the reduced graph of $\Gamma$ is the graph $\bar{\Gamma}$ in $F$ obtained by amalgamating each set of mutually parallel edges of $\Gamma$ to a single edge.

Lemma 3.2. Let $\Gamma$ be a graph in a torus such that no face of $\Gamma$ is a disk with one side, and such that, for some positive integer $n$, each vertex of $\Gamma$ has valency $6 n$. Suppose that $\Gamma$ does not contain $n+1$ mutually parallel edges. Then each parallelism class of edges of $\Gamma$ has $n$ members, and each face of the reduced graph of $\Gamma$ is a disk with 3 sides.

Proof. Let $\bar{\Gamma}$ be the reduced graph of $\Gamma$, and let $V$ and $E$ be the number of vertices and edges of $\bar{\Gamma}$.

Each vertex of $\bar{\Gamma}$ has valency $\geq(6 n / n)=6$. Therefore $2 E \geq 6 V$, giving $V \leq E / 3$.

Let $F$ be the number of disk faces of $\bar{\Gamma}$. Then, since each disk face of $\bar{\Gamma}$ has at least 3 sides, $2 E \geq 3 F$, giving $F \leq 2 E / 3$.

Finally, we have

$$
V-E+\sum \chi(f)=\chi(\text { torus })=0,
$$

where $\sum \chi(f)$ is summed over all faces $f$ of $\bar{\Gamma}$.

Hence

$$
0=V-E+\sum \chi(f) \leq V-E+F \leq \frac{E}{3}-E+\frac{2 E}{3}=0 .
$$

It follows that the above inequalities are all equalities. Thus each vertex of $\bar{\Gamma}$ has valency 6 , each face of $\bar{\Gamma}$ is a disk, and $2 E=3 F$.

We shall need the following lemma in Section 12.

Lemma 3.3. Let $\Gamma$ be a graph in an annulus such that no face of $\Gamma$ is a disk with one side, and such that, for some positive integer $n$, each vertex of $\Gamma$ has valency at least $6 n$. Then $\Gamma$ has either $n+1$ mutually parallel internal edges or $2 n$ mutually parallel boundary edges. 
Proof. Let $\bar{\Gamma}$ be the reduced graph of $\Gamma$, and let $V, E$, and $F$ be the number of vertices, edges, and disk faces of $\bar{\Gamma}$. Let $\Sigma=\sum \chi(f)$, summed over all faces of $\bar{\Gamma}$. Suppose, for a contradiction, that $\Gamma$ has neither $n+1$ parallel internal edges nor $2 n$ parallel boundary edges.

First suppose that $\Gamma$ has no boundary edges. The valency of each vertex of $\bar{\Gamma}$ is at least $(6 n / n)=6$. Hence $2 E \geq 6 V$, giving $V \leq E / 3$. Also, $2 E \geq 3 F$, giving $F \leq 2 E / 3$. Since $\bar{\Gamma}$ has a non-disk face (containing a boundary component of the annulus), we have $\Sigma<F$. Therefore

$$
0=\chi(\text { annulus })=V-E+\Sigma<V-E+F \leq E\left(\frac{1}{3}-1+\frac{2}{3}\right)=0,
$$

a contradiction.

Now suppose that $\Gamma$ has a least one boundary edge. For each vertex $v$ of $\Gamma$, let $\alpha(v)$ (resp. $\beta(v))$ be the number of incidences of internal (resp. boundary) edges at $v$, and let $\bar{\alpha}(v), \bar{\beta}(v)$ be the corresponding quantities for $\bar{\Gamma}$. By hypothesis, $\alpha(v)+\beta(v) \geq 6 n$, for all $v$. Also by hypothesis, $\bar{\alpha}(v) \geq \alpha(v) / n$ and $\bar{\beta}(v) \geq$ $\beta(v) /(2 n-1)$, for all $v$. Since $\sum \beta(v) \neq 0$ (the sum being taken over all vertices of $\Gamma)$, we have

$$
\sum \bar{\beta}(v) \geq \frac{\sum \beta(v)}{2 n-1}>\frac{\sum \beta(v)}{2 n}
$$

Hence

$$
2 E=\sum \bar{\alpha}(v)+2 \sum \bar{\beta}(v)>\frac{\sum \alpha(v)+\sum \beta(v)}{n} \geq \frac{6 n V}{n}=6 \mathrm{~V} .
$$

Thus $V>E / 3$. Also, $2 E \geq 3 F$, giving $F \leq 2 E / 3$. Therefore

$$
0=V-E+\Sigma \leq V-E+F<E\left(\frac{1}{3}-1+\frac{2}{3}\right)=0,
$$

another contradiction.

\section{InterseCtions of PUNCTURED TORI; THE GENERIC CASE}

From now on, through Section 11, we will assume that $\widehat{F}_{1}$ and $\widehat{F}_{2}$ are tori, and that $\Delta=\Delta\left(r_{1}, r_{2}\right) \geq 6$.

The following lemma is an immediate consequence of Lemma 3.1.

Lemma 4.1. If $\Delta>6$ then $F_{\alpha}$ contains $n_{\beta}+1$ mutually parallel arcs of $F_{1} \cap F_{2}$.

This motivates the following definitions. We say that $F_{\alpha}$ is excellent if it contains $n_{\beta}+1$ mutually parallel arcs of $F_{1} \cap F_{2}$ such that the corresponding permutation has more than one orbit. We say that $F_{\alpha}$ is good if it contains $n_{\beta}+1$ mutually parallel arcs of $F_{1} \cap F_{2}$ but the corresponding permutation has only one orbit. We say that $F_{\alpha}$ is bad if it does not contain $n_{\beta}+1$ mutually parallel $\operatorname{arcs}$ of $F_{1} \cap F_{2}$.

The generic situation is taken care of by the following lemma.

Lemma 4.2. If $F_{\alpha}$ is excellent then $(M, T)$ is cabled.

Proof. Without loss of generality we may assume that the labels at one end of the parallel family of arcs are $1,2, \ldots, n_{\beta}, 1$. Let the arcs be $A_{1}, A_{2}, \ldots, A_{n_{\beta}}, B_{1}$, where the subscript indicates the label at that end. (See Figure 8.)

The set of $\operatorname{arcs} \boldsymbol{A}=\left\{A_{1}, A_{2}, \ldots, A_{n_{\beta}}\right\}$ determines a permutation $\pi$. Let $\theta$ be the orbit of $\pi$ containing 1 . By hypothesis there exists another orbit $\varphi$. By Lemma 2.3 we may assume that the circles $C_{\theta}(\boldsymbol{A})$ and $C_{\varphi}(\boldsymbol{A})$ are essential in the torus $\widehat{F}_{\beta}$. 


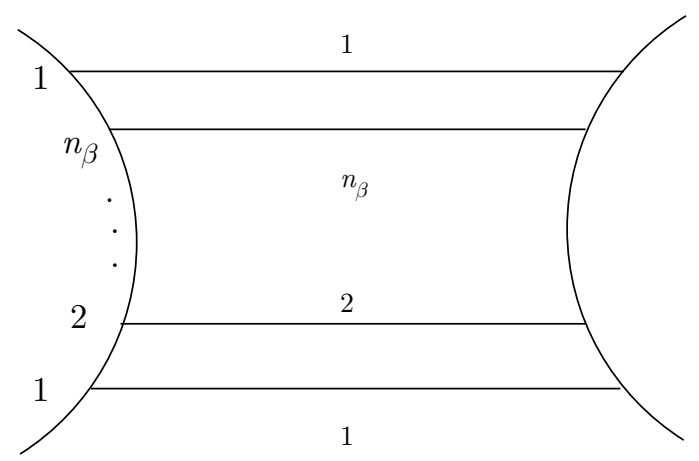

Figure 8

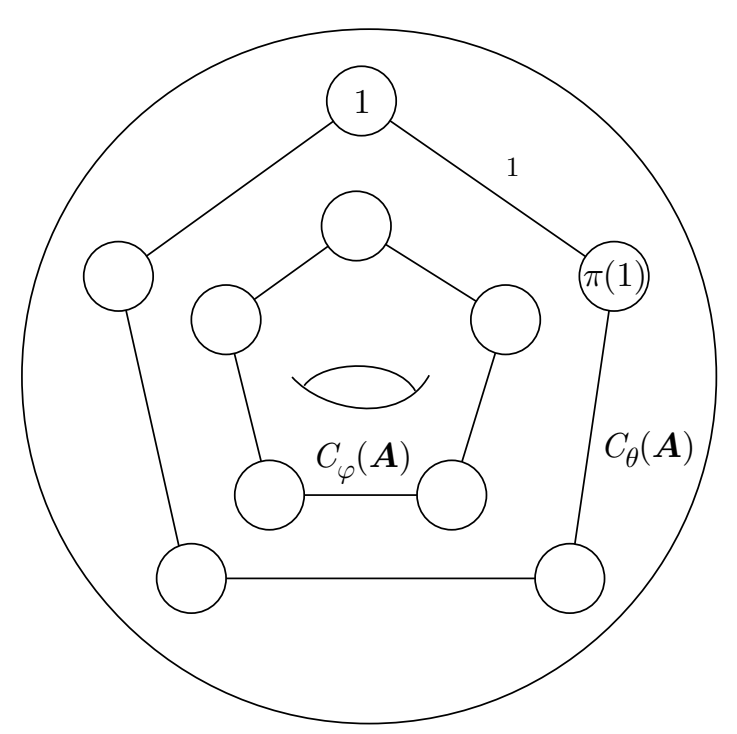

Figure 9

They are therefore parallel in $\widehat{F}_{\beta}$. Now consider the arc $B_{1}$. Since it is disjoint from $C_{\varphi}(\boldsymbol{A})$, either it is parallel to $A_{1}$ in $F_{\beta}$, or, letting $\boldsymbol{B}$ be the set of parallel $\operatorname{arcs}\left\{A_{2}, \ldots, A_{n_{\beta}}, B_{1}\right\}$, we have that $C_{\theta}(\boldsymbol{B})$ bounds a disk in $\widehat{F}_{\beta}$. (See Figure 9.) The result then follows from Lemmas 2.1 and 2.3 respectively.

We will additionally assume from now on (through Section 11) that $(M, T)$ is not cabled. Then, after Lemmas 4.1 and 4.2, exactly one of the following must hold:

(I) $F_{1}$ and $F_{2}$ are good;

(II) $\Delta=6, F_{1}$ (say) is good and $F_{2}$ is bad;

(III) $\Delta=6, F_{1}$ and $F_{2}$ are bad.

It is convenient to organize the rest of the proof along rather different lines, however, bearing in mind Lemma 2.2. Specifically, we consider the following cases: 
(A) $n_{1}=n_{2}=2$, and the two boundary components of $F_{\alpha}$ have opposite sign, $\alpha=1,2$.

(B) $n_{1}=2$, the two boundary components of $F_{1}$ have opposite sign, and all boundary components of $F_{2}$ have the same sign.

Case (B) is subdivided into four subcases as follows:

(B)(1) $n_{2}=1$,

(B)(2) $n_{2}=2$,

(B)(3) $n_{2}=3$,

(B) (4) $n_{2} \geq 4$.

(C) $\Delta=6, n_{1} \geq 3, n_{2}=2$, the two boundary components of $F_{2}$ have opposite sign, and $F_{2}$ is bad.

(D) $\Delta=6, n_{1} \geq 3$, all boundary components of $F_{2}$ have the same sign, and $F_{2}$ is bad.

(D) is subdivided into three subcases:

(D)(1) $n_{2}=1$,

(D)(2) $n_{2}=2$,

(D)(3) $n_{2} \geq 3$.

(E) $\Delta=6, F_{1}$ and $F_{2}$ bad.

By Lemma 2.2, if (I) holds then we're in either case (A) or (B), and if (II) holds then we're in either case $(\mathrm{A})$ or $(\mathrm{B})$ or $(\mathrm{C})$ or $(\mathrm{D})$.

It will turn out that the possibilities for $\left(F_{1}, F_{2} ; F_{1} \cap F_{2}\right)$ in each of these cases are as follows:

Case (A): one example with $\Delta=6$ and two examples with $\Delta=8$;

Case (B)(1): one example, with $\Delta=7$.

The combinatorics allow one example in Case (B)(1) with $\Delta=6$, and an infinite family of examples in Case (D)(1) with $\Delta=6$, but these will be shown to be topologically degenerate in Section 11.

All other cases are impossible.

Perhaps surprisingly, the hardest case to deal with appears to be (B), especially cases $(\mathrm{B})(3)$ and $(\mathrm{B})(4)$.

\section{THE NON-GENERIC CASE; PREPARATORY LEMMAS}

In Lemmas 5.1 and 5.2 we explicitly identify the reduced graph $\bar{\Gamma}_{\alpha}$ in the cases where $F_{\alpha}$ has one or two boundary components. Next, in Lemma 5.3, we establish some additional properties of the graph $\Gamma_{\alpha}$ in the case where $F_{1}$ and $F_{2}$ each have two boundary components. These will be needed in Sections 6 and 7. Finally, we note some properties of the graph $\Gamma_{\beta}$ in the case where $\Gamma_{\alpha}$ contains $n_{\beta}$ parallel edges such that the corresponding permutation has only one orbit. These are stated in Lemma 5.4. This, and Corollary 5.5, will be used in Sections 7 and 9.

Throughout, we regard two graphs in a surface as equivalent if there is a homeomorphism of the surface taking one to the other.

Lemma 5.1. If $F_{\alpha}$ has a single boundary component, then the reduced graph $\bar{\Gamma}_{\alpha}$ is a subgraph of the graph illustrated in Figure 10.

Proof. This follows immediately from the fact that the edges of $\bar{\Gamma}_{\alpha}$ are non-parallel essential loops in $\widehat{F}_{\alpha}$. 


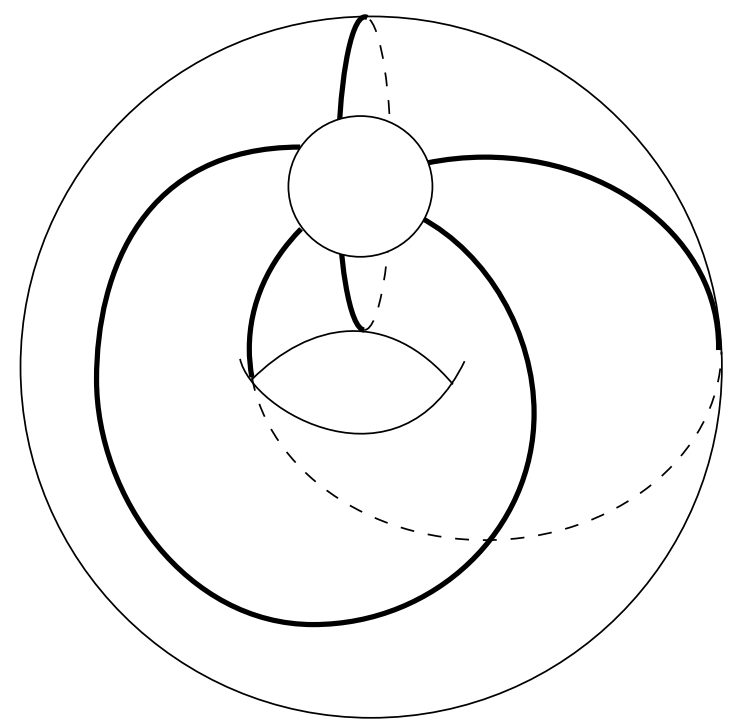

FiguRE 10

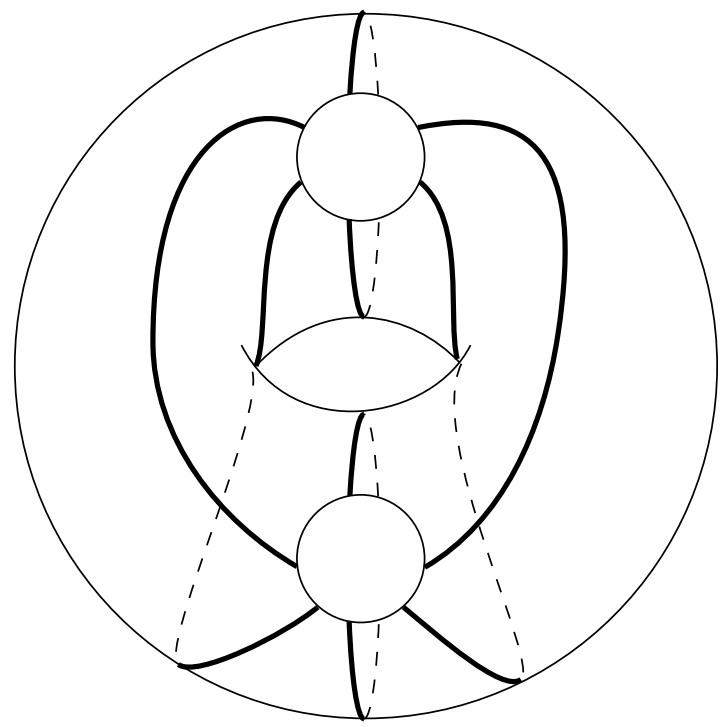

FIGURE 11

Lemma 5.2. If $F_{\alpha}$ has exactly two boundary components, then the reduced graph $\bar{\Gamma}_{\alpha}$ is a subgraph of the graph illustrated in Figure 11.

Proof. Let the vertices of $\Gamma_{\alpha}$ be $a$ and $b$. Since $a$ and $b$ have the same valency, the number of loops at $a$ is equal to the number of loops at $b$.

If this number is non-zero, there is exactly one loop at each vertex in $\bar{\Gamma}_{\alpha}$. Cutting $\widehat{F}_{\alpha}$ along one of these loops (at $b$, say) gives an annulus, as illustrated in Figure 12. Then $\bar{\Gamma}_{\alpha}$ must be a subgraph of the graph illustrated in Figure 13, as desired. 


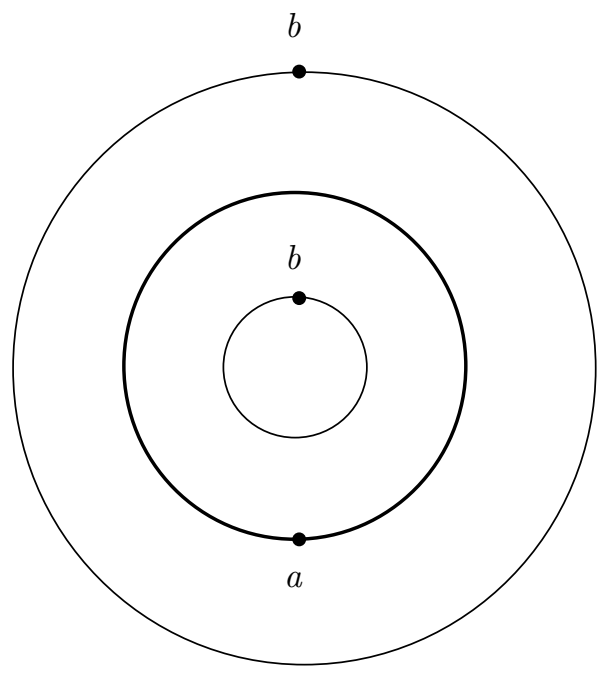

FiguRe 12

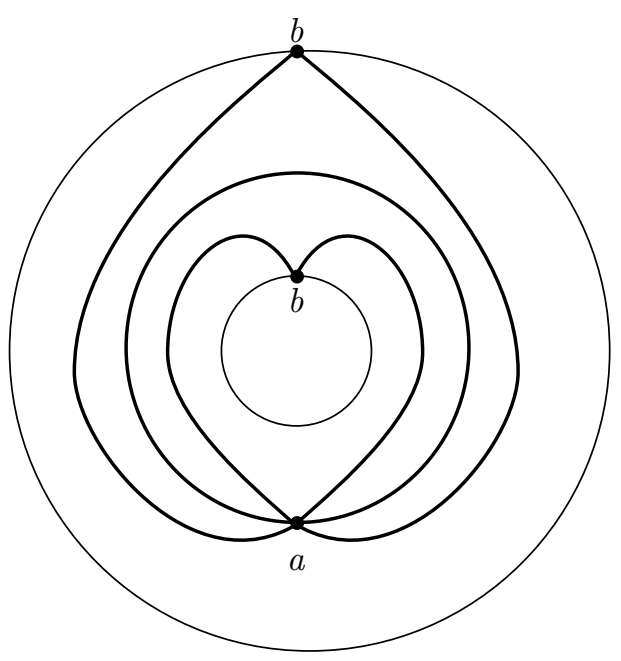

FigURE 13

If $\bar{\Gamma}_{\alpha}$ has no loops, but two non-parallel edges, then cutting $\widehat{F}_{\alpha}$ along these two edges gives an annulus as illustrated in Figure 14. Then clearly $\bar{\Gamma}_{\alpha}$ is a subgraph of either the graph illustrated in Figure 15(i) or the graph illustrated in Figure 15(ii). But these both correspond to Figure 11 (with the loops removed).

If $\Gamma$ is a graph in a torus such that $\bar{\Gamma}$ is a subgraph of the graph illustrated in Figure 10 , then $\Gamma$ is determined by the triple $\left(\beta_{1}, \beta_{2}, \beta_{3}\right)$ of non-negative integers that records the number of edges in each parallelism class, as shown in Figure 16. We say $\Gamma \cong H\left(\beta_{1}, \beta_{2}, \beta_{3}\right)$. Note that $H\left(\beta_{1}, \beta_{2}, \beta_{3}\right)$ is invariant under any permutation of the $\beta$ 's. 


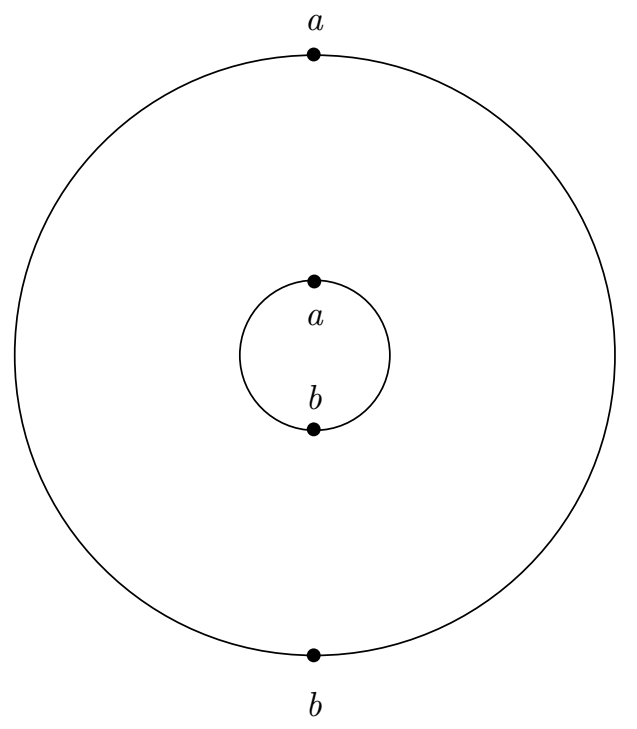

Figure 14

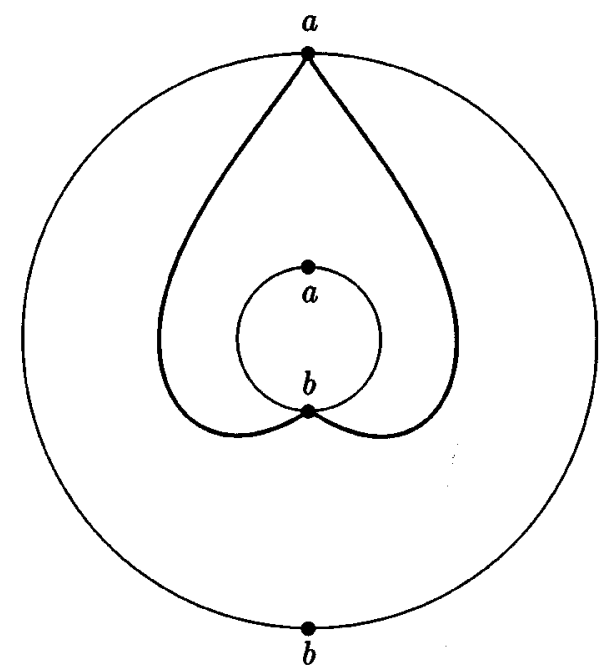

(i)

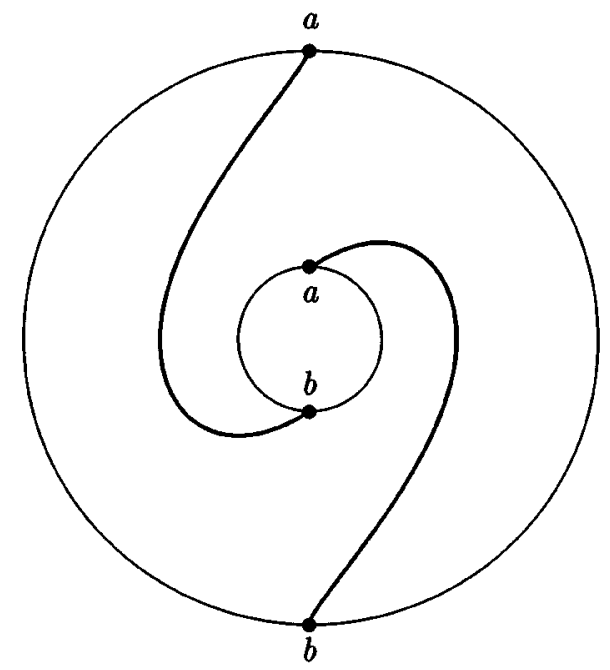

(ii)

FiguRe 15

Similarly, if $\bar{\Gamma}$ is a subgraph of the graph in Figure 11, then $\Gamma$ is determined by a quintuple $\left(\alpha_{0}, \alpha_{1}, \alpha_{2}, \alpha_{3}, \alpha_{4}\right)$ of non-negative integers, as shown in Figure 17. In this case we say $\Gamma \cong G\left(\alpha_{0}, \alpha_{1}, \alpha_{2}, \alpha_{3}, \alpha_{4}\right)$. We abbreviate $G\left(0, \alpha_{1}, \alpha_{2}, \alpha_{3}, \alpha_{4}\right)$ to $G\left(\alpha_{1}, \alpha_{2}, \alpha_{3}, \alpha_{4}\right)$. Note that

$$
\begin{aligned}
G\left(\alpha_{0}, \alpha_{1}, \alpha_{2}, \alpha_{3}, \alpha_{4}\right) & \cong G\left(\alpha_{0}, \alpha_{3}, \alpha_{4}, \alpha_{1}, \alpha_{2}\right) \cong G\left(\alpha_{0}, \alpha_{4}, \alpha_{3}, \alpha_{2}, \alpha_{1}\right) \\
& \cong G\left(\alpha_{0}, \alpha_{2}, \alpha_{1}, \alpha_{4}, \alpha_{3}\right) .
\end{aligned}
$$




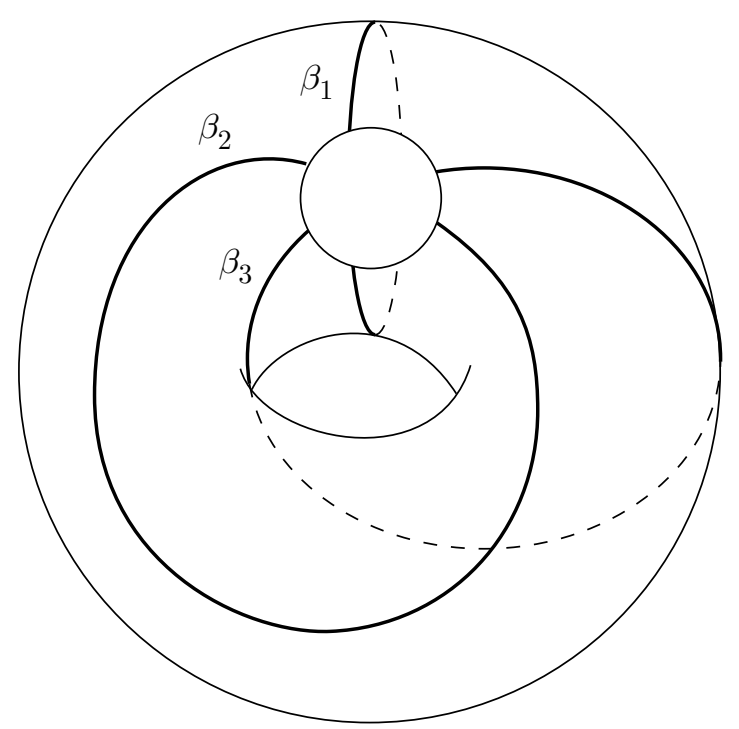

FiguRe 16

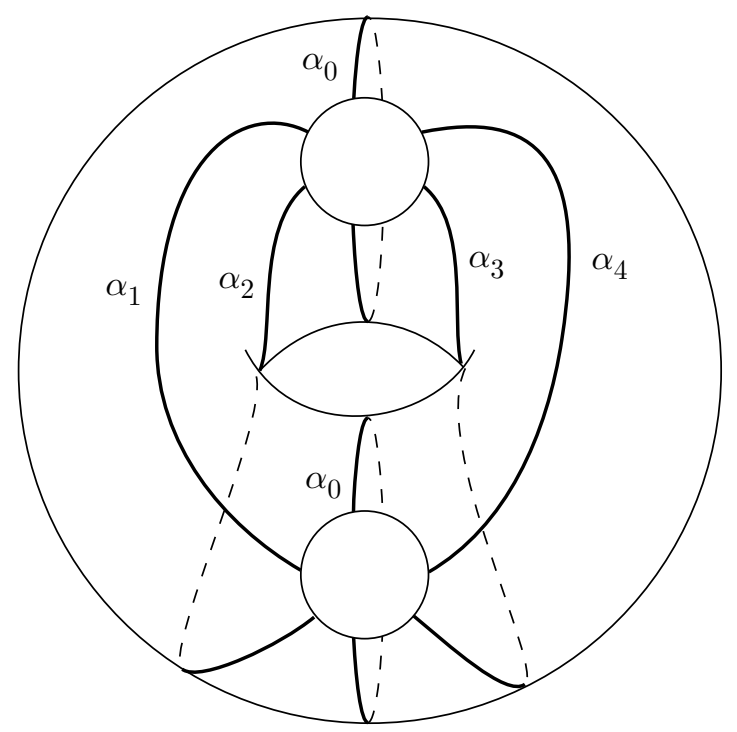

FiguRe 17

In addition, $G\left(\alpha_{1}, \alpha_{2}, \alpha_{3}, \alpha_{4}\right) \cong G\left(\alpha_{2}, \alpha_{3}, \alpha_{4}, \alpha_{1}\right)$.

For the next lemma, assume that $n_{1}=n_{2}=2$. Thus $\Gamma_{1} \cong G\left(\alpha_{0}, \alpha_{1}, \alpha_{2}, \alpha_{3}, \alpha_{4}\right)$, and similarly for $\Gamma_{2}$.

A parallel family of edges of $\Gamma_{1}$ corresponds to either loops in $\Gamma_{2}$, or edges in $\Gamma_{2}$ with distinct endpoints. Define $\varepsilon_{i}$ to be 0 or 1 according as the parallel family labelled by $\alpha_{i}$ is of the first or second kind, $i=0,1,2,3,4$. Note that $\varepsilon_{0}=1$.

Lemma 5.3. Suppose that $n_{1}=n_{2}=2$. Then 


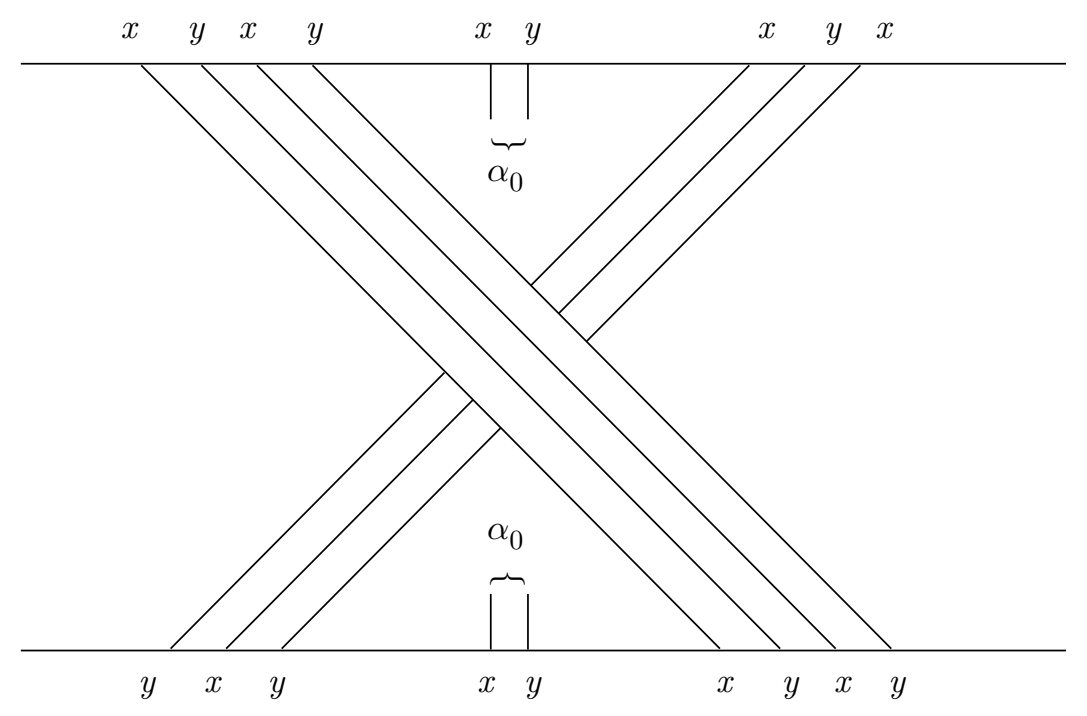

FiguRE 18

(i) if $\varepsilon_{i}=0$ then $\alpha_{i} \leq 2$;

(ii) if $\varepsilon_{i}=1$ then $\alpha_{i} \leq 4$;

(iii) $\alpha_{i}+\varepsilon_{i} \equiv \alpha_{j}+\varepsilon_{j}(\bmod 2), i, j=1,2,3,4$.

Proof. (i) and (ii) follow respectively from the facts that $\Gamma_{2}$ has at most 2 parallelism classes of loops, and at most 4 parallelism classes of edges with distinct endpoints, together with Lemma 2.1.

To prove (iii), first consider the parallelism classes of edges of $\Gamma_{1}$ corresponding to $i=2$ and 3 . The way the endpoints of the corresponding arcs lie on the two boundary components of $F_{1}$ is illustrated in Figure 18. Let the boundary components of $F_{2}$ be $x$ and $y$. Since the labels $x, y$ alternate around the boundary components of $F_{1}$, the fact that $\alpha_{2}+\varepsilon_{2} \equiv \alpha_{3}+\varepsilon_{3}(\bmod 2)$ easily follows by inspection. (E.g., Figure 18 explicitly illustrates the case $\alpha_{2} \equiv 0(\bmod 2), \varepsilon_{2}=0, \alpha_{3}=1$ $(\bmod 2), \varepsilon_{1}=1$, when $\alpha_{0}$ is even.) Similarly $\alpha_{4}+\varepsilon_{4} \equiv \alpha_{1}+\varepsilon_{1}(\bmod 2)$.

The arrangement of the parallelism classes of edges corresponding to $i=1$ and 2 corresponds to Figure 18 with $\alpha_{0}=0$. Hence we get $\alpha_{1}+\varepsilon_{1} \equiv \alpha_{2}+\varepsilon_{2}(\bmod 2)$. Similarly $\alpha_{3}+\varepsilon_{3} \equiv \alpha_{4}+\varepsilon_{4}(\bmod 2)$.

Let $\Gamma$ be a graph in a torus and let $x, y$ be vertices of $\Gamma$. Then $\nu(x, y)$ will denote the number of edges in the reduced graph $\bar{\Gamma}$ that join $x$ and $y$.

Lemma 5.4. Suppose that $\Gamma_{\alpha}$ contains $n_{\beta}$ parallel edges such that the corresponding permutation $\pi$ has only one orbit. Then

(i) $\nu(x, \pi(x)) \leq 5$ for all vertices $x$ of $\Gamma_{\beta}$;

(ii) if $\nu(x, \pi(x)) \geq 3$, then $\nu(y, \pi(y))=1$ for $y \neq x, \pi(x)$ or $\pi^{-1}(x)$.

Proof. Cutting the torus $\widehat{F}_{\beta}$ along the edges of $\Gamma_{\beta}$ corresponding to the $n_{\beta}$ parallel edges in $\Gamma_{\alpha}$ gives an annulus $N$, by Lemma 2.3 (see Figure 19).

(i) We consider how any additional edges of $\bar{\Gamma}_{\beta}$ that join $x$ and $\pi(x)$ can lie in $N$. Such an edge either has both endpoints on the same component of $\partial N$ (type I), or joins the two components of $\partial N$ (type II). There are at most two edges of type I 


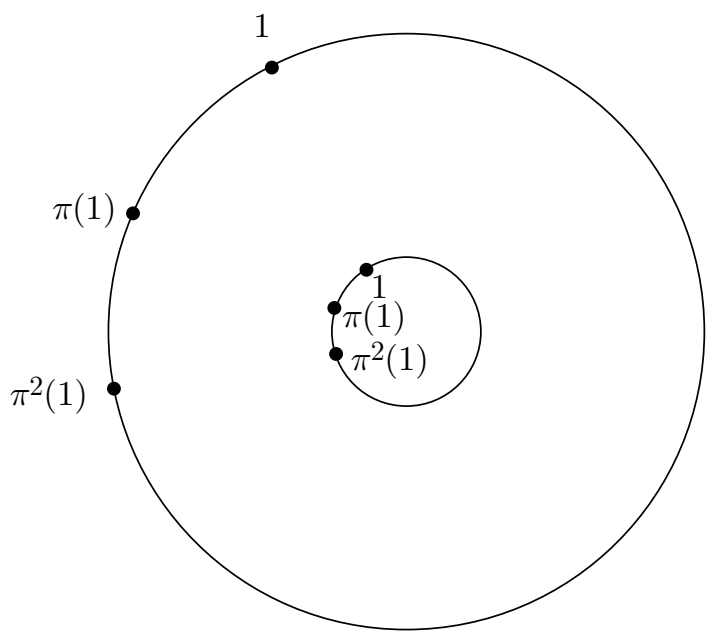

FIGURE 19

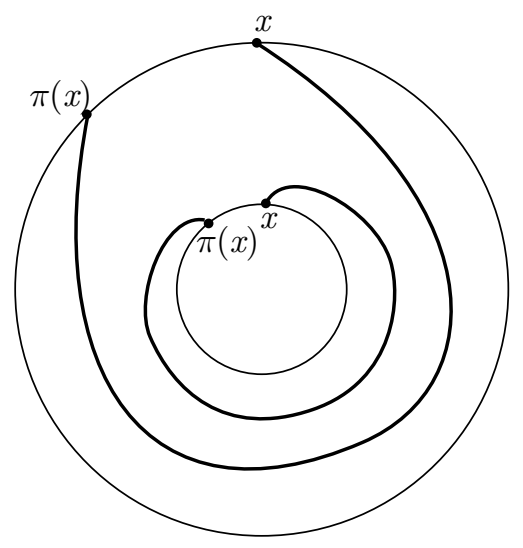

FIGURE 20

(one for each component of $\partial N$ ); see Figure 20. Also there are at most two of type II; see Figures 21(i) and 21(ii) for the only two possibilities, up to symmetry. Hence $\nu(x, \pi(x)) \leq 5$.

(ii) Note that since the assertion is vacuously true if $n_{\beta} \leq 3$, we may assume $n_{\beta} \geq 4$. Suppose $\nu(x, \pi(x)) \geq 3$. Then we have two edges of $\bar{\Gamma}_{\beta}$ joining $x$ and $\pi(x)$ in the annulus $N$, as described in (i). Up to symmetry, the only possibilities for such a pair of edges are illustrated in Figures 20, 21 and 22. In each case it is clear that if $\{y, \pi(y)\} \cap\{x, \pi(x)\}=\emptyset$ then $\nu(y, \pi(y))=1$.

Corollary 5.5. Suppose that $n_{\beta} \geq 4$. Then the number of edges in any parallelism class in $\Gamma_{\alpha}$ is at most $2 n_{\beta}$.

Proof. Suppose that $\Gamma_{\alpha}$ contains $2 n_{\beta}+1$ mutually parallel edges. Without loss of generality, assume that the labels at one end of the parallelism class are $1,2, \ldots, n_{\beta}$, $1,2, \ldots, n_{\beta}, 1$. By Lemma 4.2 the corresponding permutation $\pi$ has only one orbit. 


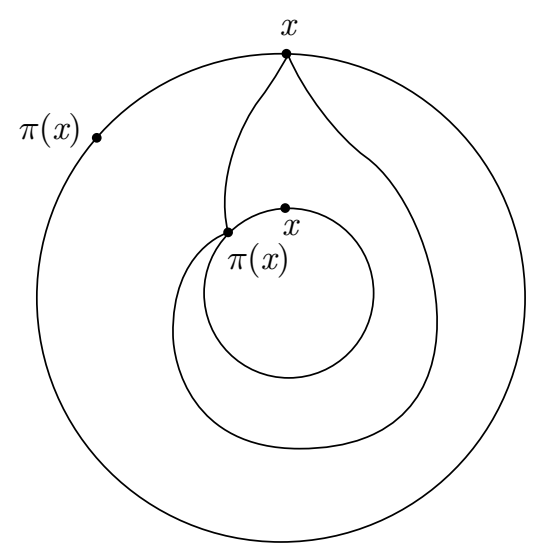

(i)

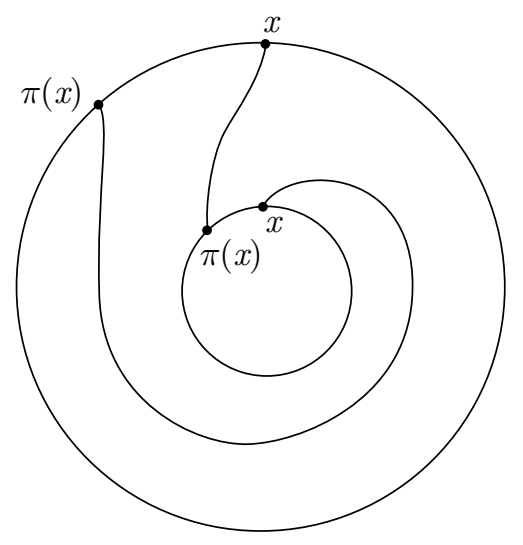

(ii)

FiguRe 21

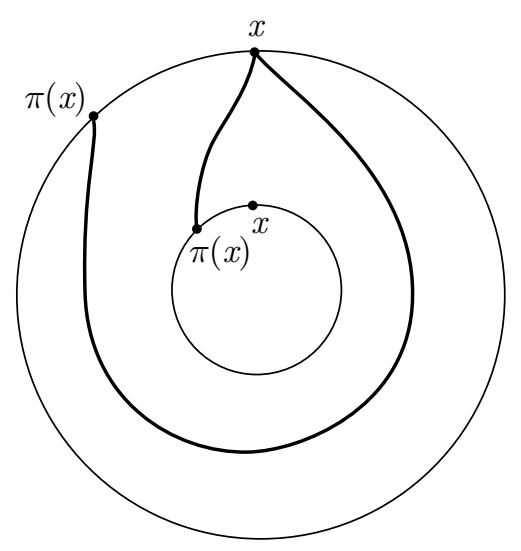

FiguRe 22

By Lemma 2.1, $\nu(x, \pi(x)) \geq 2$ for all vertices $x$ of $\Gamma_{\beta}$, and $\nu(1, \pi(1)) \geq 3$. But since $n_{\beta} \geq 4$, there exists $x$ such that $x \neq 1, \pi(1)$, or $\pi^{-1}(1)$. We thus get a contradiction to Lemma 5.4(ii).

\section{Case (A)}

In this section we treat the case where $n_{1}=n_{2}=2$, and the two boundary components of $F_{\alpha}$ have opposite sign, $\alpha=1,2$. Call the boundary components of $F_{\alpha}$ (equivalently, the vertices of $\left.\Gamma_{\alpha}\right)+$ and $-, \alpha=1,2$.

By Lemma $5.2, \Gamma_{1} \cong G\left(\alpha_{0}, \alpha_{1}, \alpha_{2}, \alpha_{3}, \alpha_{4}\right)$, and $\Gamma_{2} \cong G\left(\beta_{0}, \beta_{1}, \beta_{2}, \beta_{3}, \beta_{4}\right)$.

Lemma 6.1.

(i) $2 \alpha_{0}+\sum_{i=1}^{4} \alpha_{i}=2 \beta_{0}+\sum_{i=1}^{4} \beta_{i}=2 \Delta$; 
(ii) $2 \alpha_{0}=\sum_{i=1}^{4} \beta_{i}, \quad 2 \beta_{0}=\sum_{i=1}^{4} \alpha_{i}$;

(iii) $\alpha_{0}, \beta_{0} \leq 4$;

(iv) $\alpha_{i}, \beta_{i} \leq 2, \quad i=1,2,3,4$.

(v) $\alpha_{i} \equiv \alpha_{j}(\bmod 2), \quad i, j=1,2,3,4$, and similarly for the $\beta$ 's.

Proof. (i) follows by considering the total number of edges of $\Gamma_{\alpha}$.

By the parity rule, loops in $\Gamma_{\alpha}$ correspond precisely to edges in $\Gamma_{\beta}$ with distinct endpoints. This gives (ii).

(iii) and (iv) follow from Lemma 5.3, (i) and (ii).

(v) follows from Lemma 5.3 (iii), since here $\varepsilon_{i}=0, i=1,2,3,4$.

Corollary 6.2. Either $\alpha_{0} \geq \Delta / 2$ or $\beta_{0} \geq \Delta / 2$.

Proof. Lemma 6.1, (i) and (ii).

Corollary 6.3. $\Delta \leq 8$.

Proof. Corollary 6.2 and Lemma 6.1 (iii).

Lemma 6.4. (i) If $\Delta=8$ then $\Gamma_{1} \cong \Gamma_{2} \cong G(4,2,2,2,2)$.

(ii) $\Delta=7$ is impossible.

(iii) If $\Delta=6$ then $\Gamma_{1}$ (say) $\cong G(4,2,0,0,2)$ and $\Gamma_{2} \cong G(2,2,2,2,2)$.

Proof. (i) By Corollary 6.2 and Lemma 6.1 (iii), $\alpha_{0}$ (say) = 4. Hence by Lemma 6.1, (i) and (iv), $\alpha_{1}=\alpha_{2}=\alpha_{3}=\alpha_{4}=2$. Lemma 6.1 (ii) (and (iv)) now gives the same conclusion for the $\beta$ 's.

(ii) By Corollary 6.2 and Lemma 6.1 (iii), $\alpha_{0}$ (say) $=4$. Hence $\beta_{0}=3$ (by Lemma 6.1, (i) and (ii)), and so (by Lemma 6.1 (iv)) $\Gamma_{2} \cong G(3,2,2,2,2)$.

Let $A, B, C, D$ be the edges of $\Gamma_{2}$ indicated in Figure 23. These are the 4 loops at the vertex + (say) in $\Gamma_{1}$, and hence they are all parallel in $\Gamma_{1}$. But this is impossible by Lemma 2.5. More precisely, if $X$ is an edge of $\Gamma_{2}$ joining vertices + and -, let $X^{+}, X^{-}$denote the endpoints of $X$ at + and - respectively. If two such edges $X, Y$ are parallel in $\Gamma_{1}$, then by either (i) or (ii) of Lemma 2.5 we must have $\delta_{2}\left(X^{+}, Y^{+}\right)=\delta_{2}\left(X^{-}, Y^{-}\right)$. One readily verifies that the only pairs of edges among $A, B, C$, and $D$ permitted to be parallel in $\Gamma_{1}$ by this condition are $A, B$ and $C, D$. For example, for suitable choice of orientation of $F_{2}$, we have $\delta_{2}\left(A^{+}, C^{+}\right)=8$, while $\delta_{2}\left(A^{-}, C^{-}\right)=6$.

(iii) By Corollary 6.2 and Lemma 6.1 (iii), $\alpha_{0}$ (say) $=3$ or 4 . We consider these two cases separately.

CASE 1. $\alpha_{0}=3$. By Lemma $6 \cdot 1$, (i), (iv), and (v), $\Gamma_{1} \cong G(3,2,2,2,0)$. Let $A, B, C$ be the edges of $\Gamma_{1}$ indicated in Figure 24. These are loops at the vertex + (say) in $\Gamma_{2}$, and hence are parallel in $\Gamma_{2}$. But as in (ii) above, it is easy to check that $C$ cannot be parallel to $A$ or $B$ in $\Gamma_{2}$ by Lemma 2.5(i) or (ii).

CASe 2. $\alpha_{0}=4$. By Lemma 6.1, (i), (ii), (iv) and (v), $\Gamma_{1} \cong G(4,2,0,0,2)$, $G(4,2,2,0,0)$, or $G(4,1,1,1,1)$. We shall show that the last two possibilities cannot occur.

First note that by Lemma 6.1 , (i) and (ii), $\Gamma_{2} \cong G(2,2,2,2,2)$. Let $A_{1}, A_{2}, \ldots$, $A_{6}$ be the edges of $\Gamma_{2}$ with label + at vertex + of $\Gamma_{2}$, numbered cyclically around that vertex in such a way that $A_{1}$ and $A_{4}$ are loops (see Figure 25). Since $d=d_{12}=$ $d_{21}=1$ (see Section 2), these edges appear in the same order around vertex + of $\Gamma_{1}$. 


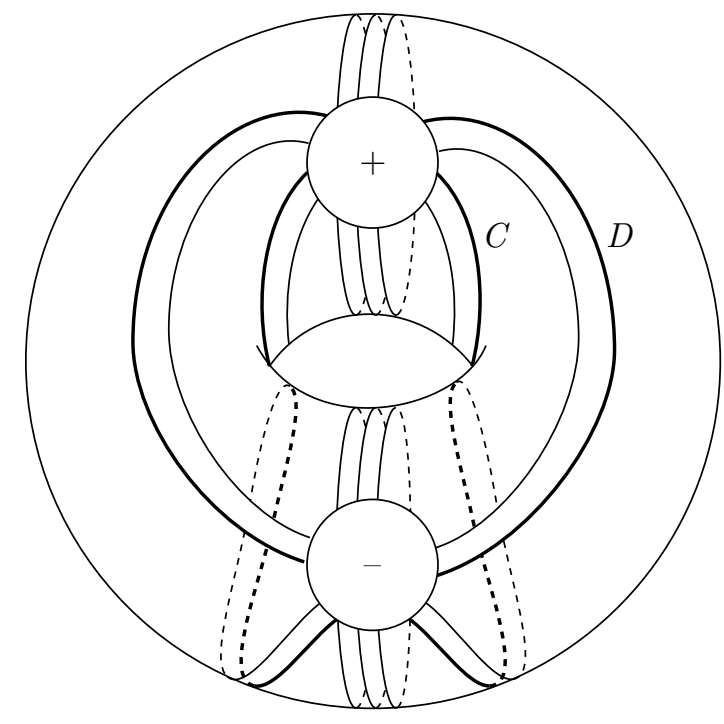

FiguRE 23

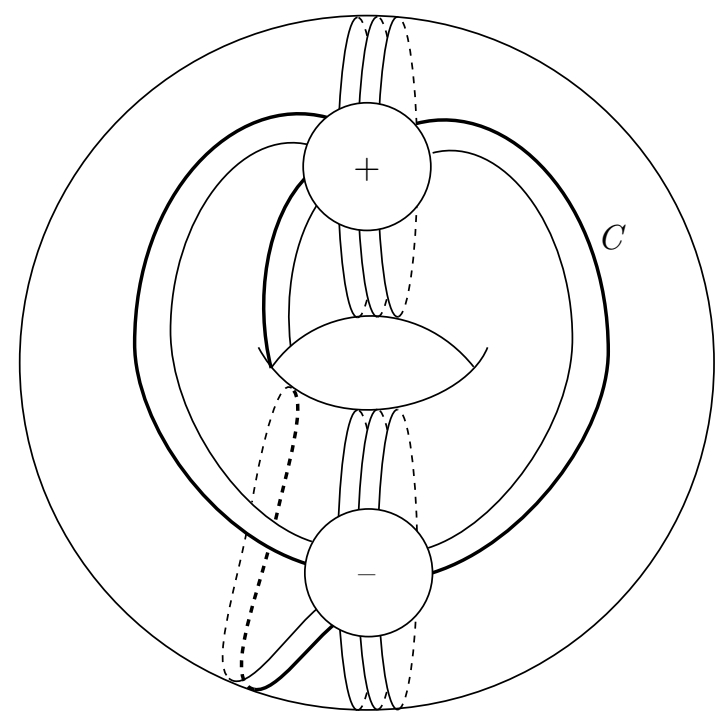

FigURE 24

Since $A_{1}, A_{4}$ join distinct vertices of $\Gamma_{1}$, this implies that $\alpha_{1}+\alpha_{2} \neq 0 \neq \alpha_{3}+\alpha_{4}$. This rules out the possibility that $\Gamma_{1} \cong G(4,2,2,0,0)$.

To rule out the possibility $\Gamma_{1} \cong G(4,1,1,1,1)$, consider the loops in $\Gamma_{1}$. The loops at vertex + correspond to the edges $A_{2}, A_{3}, A_{5}, A_{6}$ of $\Gamma_{2}$. Note that each of these edges is parallel to another edge of $\Gamma_{2}$ that corresponds to a loop in $\Gamma_{1}$ at vertex - . Let $X$ be the loop in $\Gamma_{1}$ at vertex + shown in Figure 26. Let $X( \pm)$ be the endpoint of $X$ with label \pm . Then (for suitable choice of orientation of boundary component + of $F_{1}$ ) we see that $\tau_{1}(X(+), X(-))=3$. Let $Y$ be the edge of $\Gamma_{2}$ 


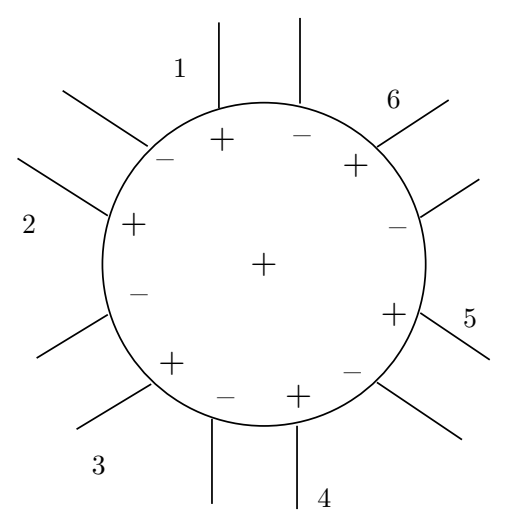

FiguRE 25

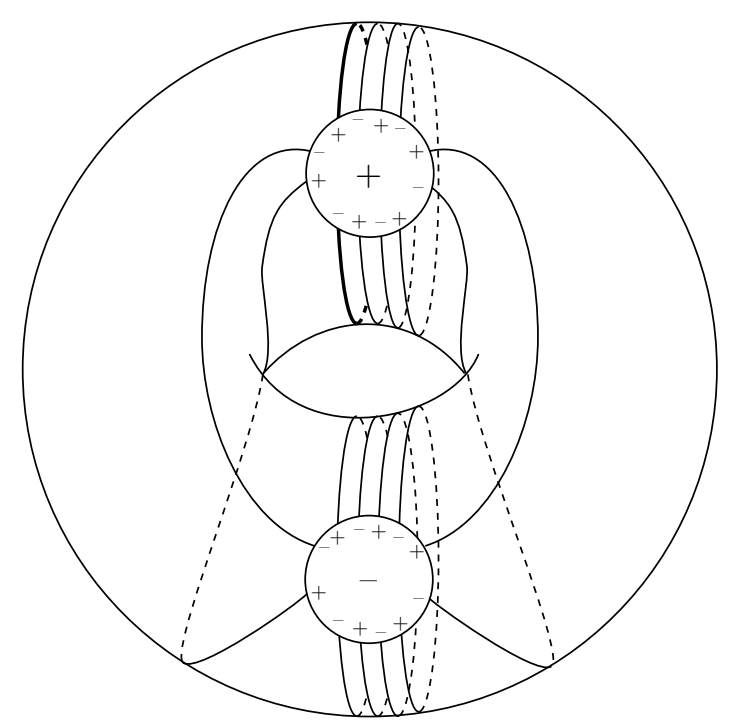

FIGURE 26

that is parallel to $X$ in $\Gamma_{2}$. Then $Y$ is a loop in $\Gamma_{1}$ at vertex -. But, recalling that boundary components + and - of $F_{1}$ are of opposite sign, it is easy to verify that no such loop has $\tau_{1}(Y(+), Y(-))=3$. This contradicts Lemma 2.5(iii).

The possibilities allowed by Lemma 6.4 do in fact occur. We describe the identification between the edges of $\Gamma_{1}$ and $\Gamma_{2}$ in these cases.

First consider the case given in Lemma 6.4(iii), with $\Delta=6$. Let $A_{1}, A_{2}, \ldots, A_{6}$ be the edges of $\Gamma_{2}$ with label + at vertex + of $\Gamma_{2}$, as in Figure 25. Since here $d=1$, these edges appear in the same order around vertex + of $\Gamma_{1}$. Recalling that loops in $\Gamma_{\alpha}$ correspond to edges with distinct endpoints in $\Gamma_{\beta}$, we see that the edges $A_{1}, A_{2}, \ldots, A_{6}$ must appear around vertex + of $\Gamma_{1}$ as shown in Figure 27. It is easy to check that this determines the identification between all the edges of $\Gamma_{1}$ and $\Gamma_{2}$, and gives rise to the identification pattern $P(6)$ shown in Figures 28(i) and (ii). 


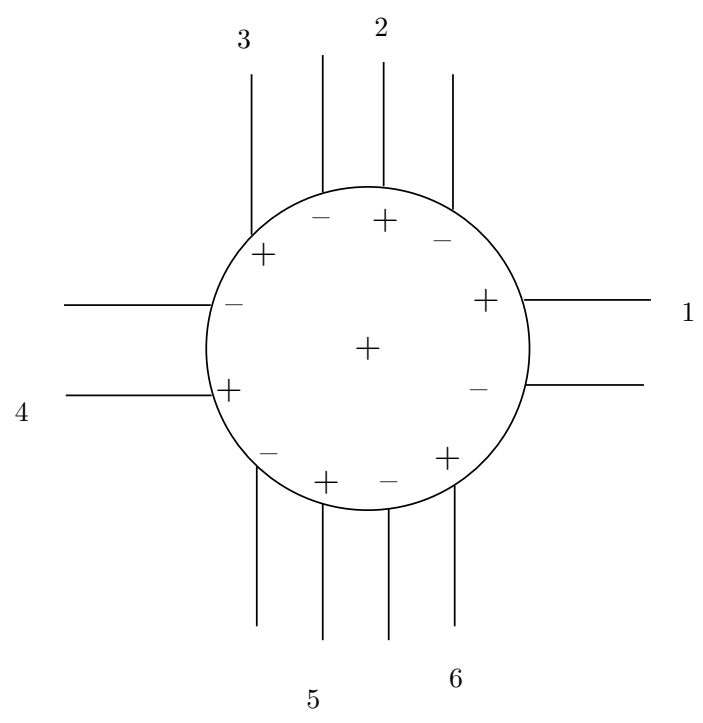

FIGURE 27

(In these figures the edges $A_{1}, A_{2}, A_{3}, A_{4}, A_{5}, A_{6}$ have been relabeled $W, C, A, Y, B$, and $D$ respectively.)

In the case $\Delta=8$ (Lemma $6.4(\mathrm{i})$ ), let $A_{1}, A_{2}, \ldots, A_{8}$ be the edges of $\Gamma_{2}$ with label + at vertex + of $\Gamma_{2}$, numbered cyclically in such a way that $A_{3}, A_{4}, A_{7}$, and $A_{8}$ are loops (see Figure 29). There are now two possibilities for $d=d_{21}$, namely $d=1$ and $d=3$, giving rise to the two arrangements of $A_{1}, A_{2}, \ldots, A_{8}$ around vertex + of $\Gamma_{1}$ shown in Figures 30(i) and (ii). Again, it is straightforward to check that each case leads to a unique possibility for the identification between the edges of $\Gamma_{1}$ and $\Gamma_{2}$. The corresponding identification patterns $P(8)_{1}$ and $P(8)_{2}$ are shown in Figures 31 and 32; the graph $\Gamma_{2}$ in both cases is shown in Figure 31, while the graphs $\Gamma_{1}$ for $P(8)_{1}$ and $P(8)_{2}$ are shown in Figures 32(i) and (ii) respectively. (In these figures, $A_{1}, A_{2}, \ldots, A_{8}$ have been relabeled $S, U, D, B, W, Y, A, C$.)

We have thus shown that in Case (A), the only possibilities for $F_{1}, F_{2}$ are given by the identification patterns $P(6), P(8)_{1}$ and $P(8)_{2}$.

\section{CAse (B)}

In this section we treat the case where $n_{1}=2$, the two boundary components of $F_{1}$ have opposite sign, and all the boundary components of $F_{2}$ have the same sign. Call the boundary components of $F_{1}$ (the vertices of $\Gamma_{1}$ ) + and - . Since all the boundary components of $F_{2}$ have the same sign, the parity rule implies that there are no loops in $\Gamma_{1}$. Hence $\Gamma_{1} \cong G\left(\alpha_{1}, \alpha_{2}, \alpha_{3}, \alpha_{4}\right)$.

We distinguish the four cases $n_{2}=1, n_{2}=2, n_{2}=3$, and $n_{2} \geq 4$.

CASE $(1) . n_{2}=1$. Here $\Gamma_{2} \cong H\left(\beta_{1}, \beta_{2}, \beta_{3}\right)$. Note that

$$
\sum_{i=1}^{4} \alpha_{i}=\sum_{i=1}^{3} \beta_{i}=\Delta .
$$


C. McA. GORDON

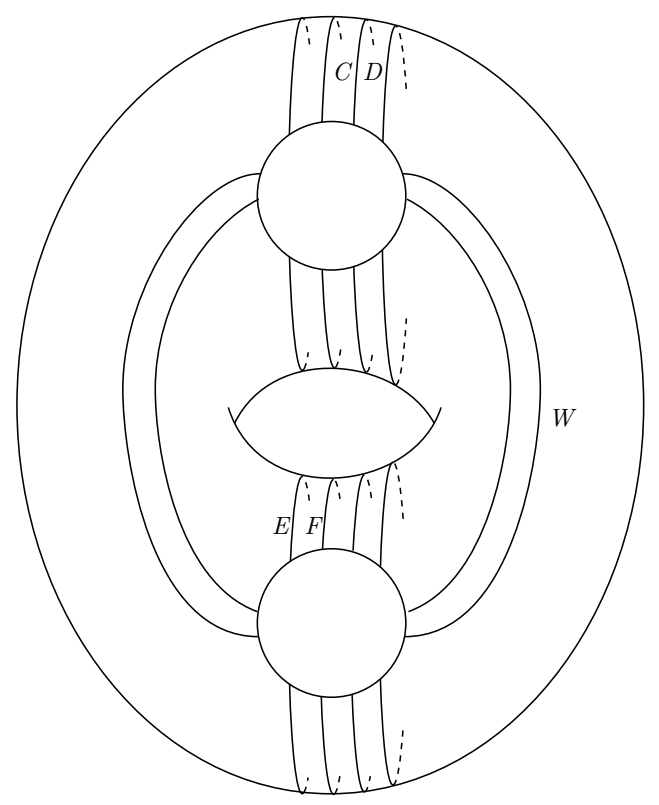

(i)

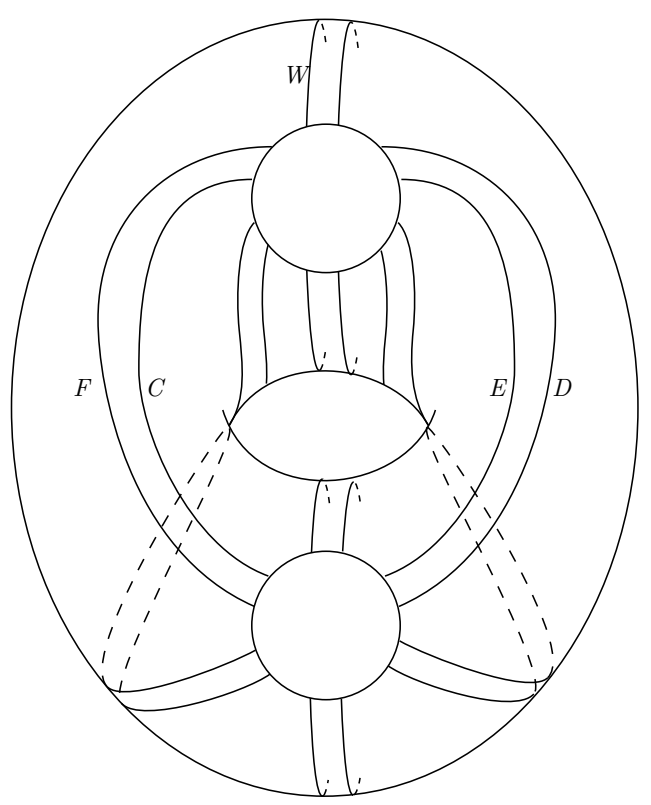

(ii)

Figure 28 
6

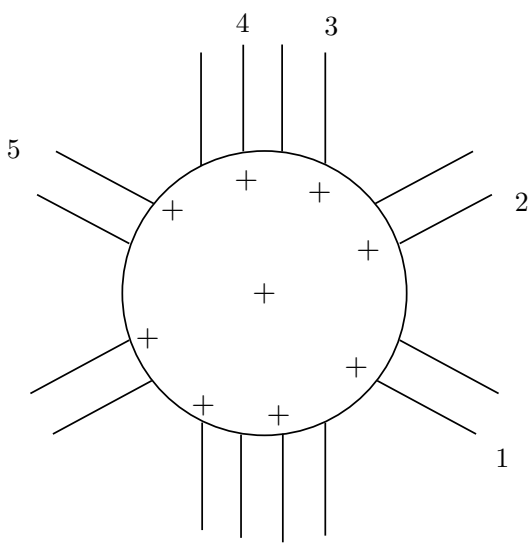

$7 \quad 8$

FiguRe 29

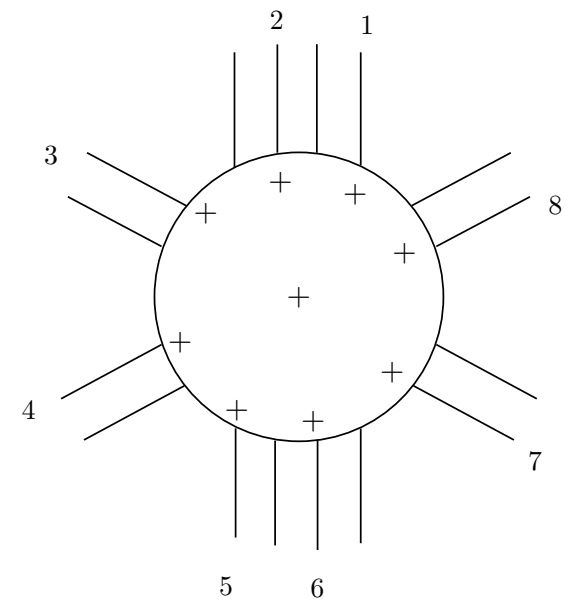

(i)

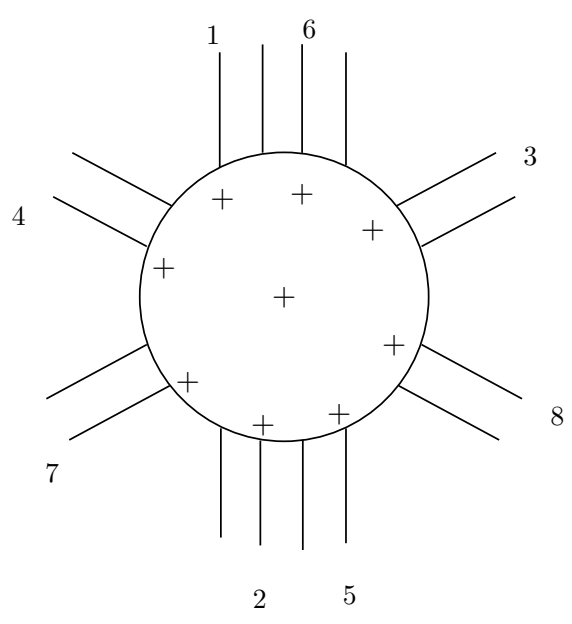

(ii)

FiguRE 30

Lemma 7.1. (i) $\beta_{i} \leq 4, \quad i=1,2,3$;

(ii) $\beta_{1} \equiv \beta_{2} \equiv \beta_{3}(\bmod 2)$.

Proof. (i) follows from the fact that there are at most 4 parallelism classes of edges in $\Gamma_{1}$, together with Lemma 2.1.

Since the labels at the endpoints of a loop in any parallelism class are distinct, we see that $\beta_{i} \neq 0$ implies that $\beta_{j}+\beta_{k}$ is even $(\{i, j, k\}=\{1,2,3\})$. Since at most one $\beta_{i}$ is zero by (i), this proves (ii).

The only possibilities for $\Gamma_{2}$ allowed by Lemma 7.1 with $\Delta \geq 6$ are the following, where $d=d_{21}$ : 


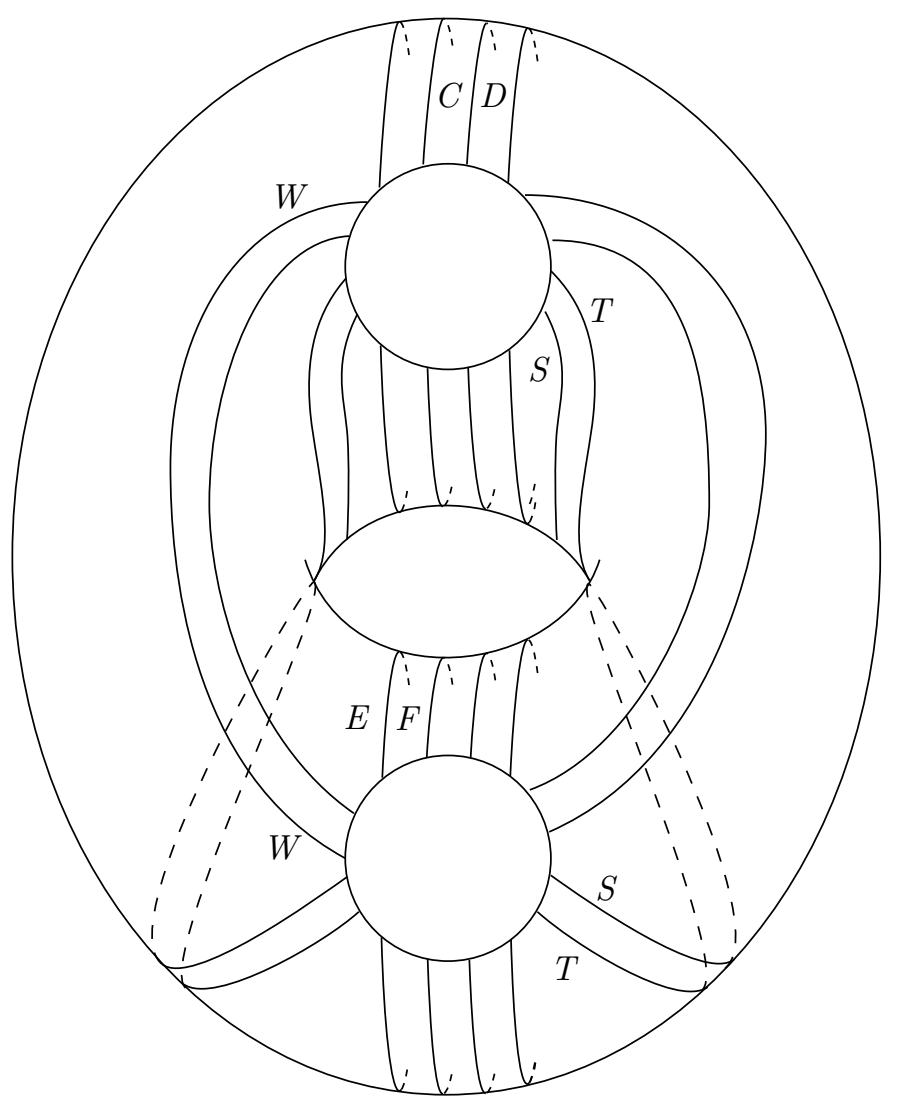

FiguRe 31

$$
\begin{array}{lll}
\Delta=12, & H(4,4,4), & d=1 \text { or } 5 . \\
\Delta=10, & H(4,4,2), & d=1 \text { or } 3 . \\
\Delta=9, & H(3,3,3), & d=1,2 \text { or } 4 . \\
\Delta=8, & H(4,4,0) \text { or } H(4,2,2), & d=1 \text { or } 3 . \\
\Delta=7, & H(3,3,1), & d=1,2, \text { or } 3 . \\
\Delta=6, & H(4,2,0) \text { or } H(2,2,2), & d=1 .
\end{array}
$$

Consider the case $\Delta=12$. Let the edges of $\Gamma_{2}$ be $A_{1}, A_{2}, \ldots, A_{12}$, numbered around the vertex of $\Gamma_{2}$ according to the position of the end with label + . See Figure 33. These edges occur around the vertex + of $\Gamma_{1}$ in the order $A_{1}, A_{1+d}$, $A_{1+2 d}, \ldots$. Here, $d=1$ or 5 .

First suppose that $d=1$. Then (interpreting subscripts modulo 12) no consecutive pair $A_{i}, A_{i+1}$ can be parallel in $\Gamma_{1}$, either because they are parallel in $\Gamma_{2}$ (and by Lemma 2.1), or because their endpoints violate Lemma 2.5(i) (or, equivalently here, Lemma 2.5(iii)). But this contradicts the form of $\Gamma_{1}$. Similarly, in the case $d=5$, we see that no pair $A_{i}, A_{i+5}$ can be parallel in $\Gamma_{1}$.

The other cases are handled in the same way, by examining the arrangement of the edges $A_{1}, \ldots, A_{\Delta}$ around the vertex of $\Gamma_{2}$. For instance, in the case $\Delta=10$, 


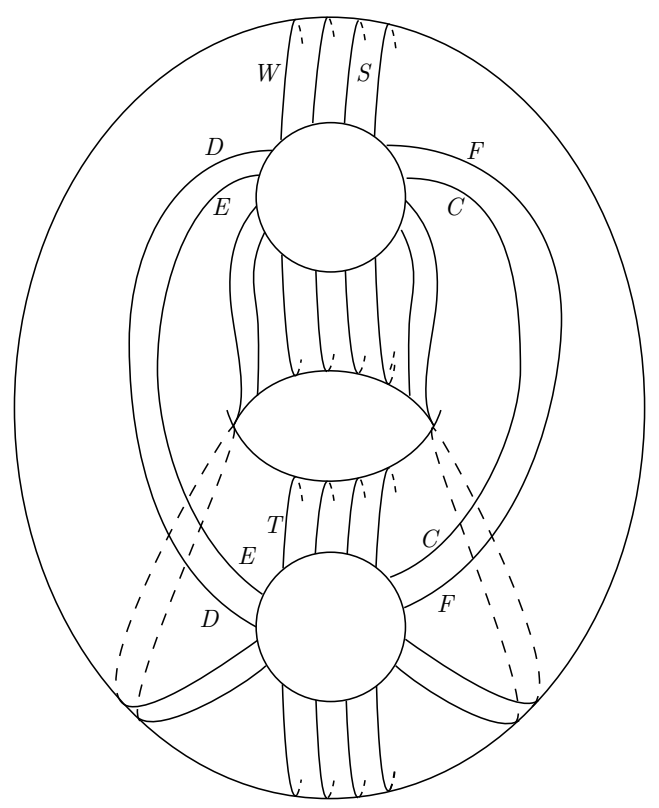

(i)

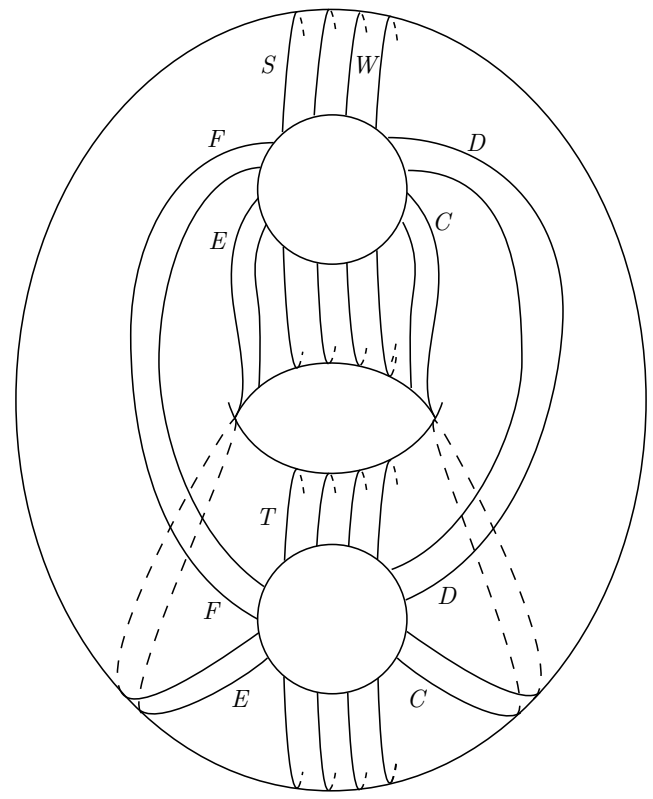

(ii)

FiguRE 32 


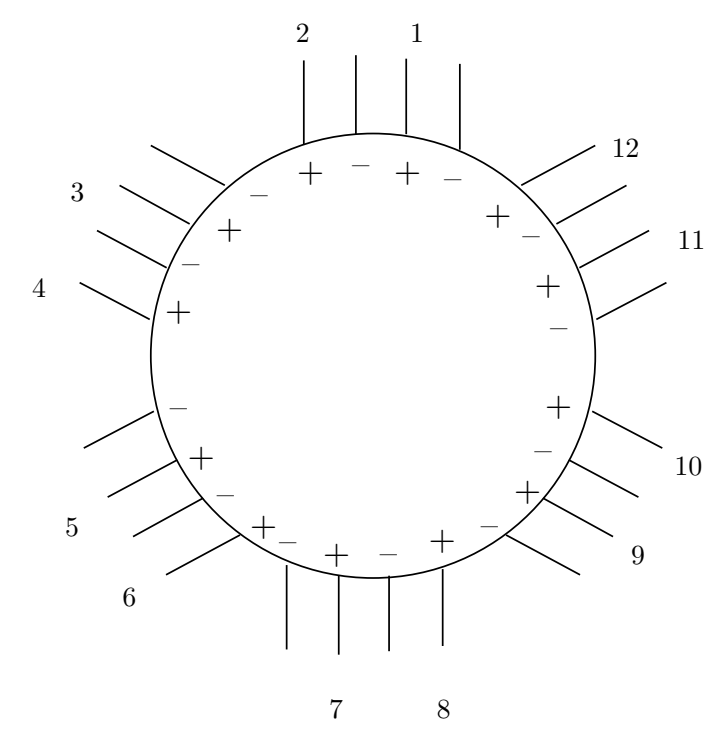

FiguRE 33

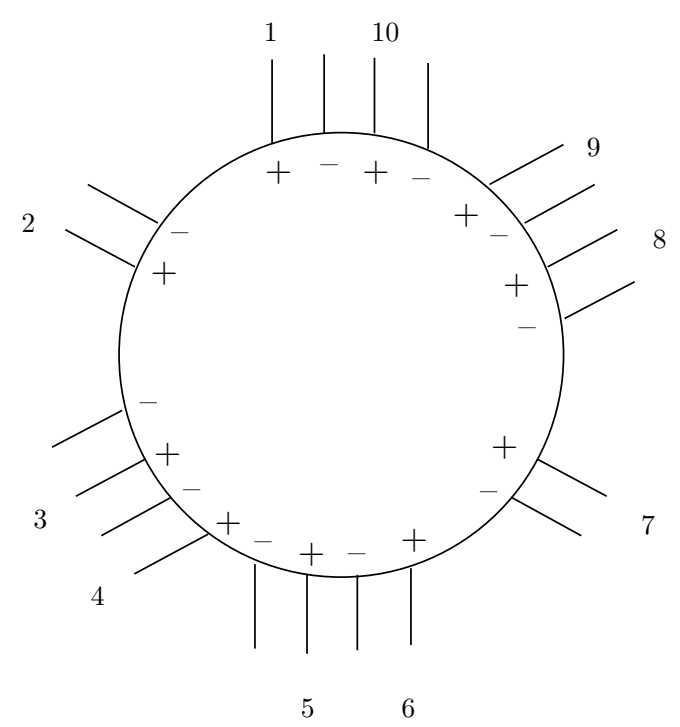

FIGURE 34

$d=3$, we see using Lemma 2.5(i) that the only pairs $A_{i}, A_{i+3}$ that can be parallel in $\Gamma_{1}$ are $A_{1}$ and $A_{4}, A_{10}$ and $A_{3}, A_{6}$ and $A_{9}$, and $A_{5}$ and $A_{8}$. See Figure 34. Since $\Gamma_{1}$ has at most 4 parallelism classes of edges, this is a contradiction.

One readily checks that the only possibilities not ruled out by this means are $\Delta=7, d=2$, and $\Delta=6, \Gamma_{2} \cong H(2,2,2)$. These do in fact occur, with $\Gamma_{1} \cong$ $G(2,1,2,2)$ and $G(3,0,0,3)$ respectively. The (unique) corresponding identification patterns of the edges of $\Gamma_{1}$ and $\Gamma_{2}, P(7)$ and $P(6)_{2}$, are shown in Figures 35 and 
36 respectively. We shall see later, however, in Section 11, that the case $P(6)_{2}$ is topologically degenerate.

CASE $(2) . n_{2}=2$. Here $\Gamma_{2} \cong G\left(\beta_{0}, \beta_{1}, \beta_{2}, \beta_{3}, \beta_{4}\right)$. Call the boundary components of $F_{2}$ (the vertices of $\Gamma_{2}$ ) $x$ and $y$.

Lemma 7.2. $\Delta \leq 5$.

Proof. Note that $\sum_{i=1}^{4} \alpha_{i}=2 \Delta$. Also, since $n_{1}=n_{2}=2$, Lemma 5.3 applies. In particular, $\alpha_{i} \leq 4, i=1,2,3,4$. Hence $\Delta \leq 8$. It remains to consider the cases $\Delta=8, \Delta=7$, and $\Delta=6$.

$\Delta=8$ is impossible. Here $\sum_{i=1}^{4} \alpha_{i}=16$. Hence, by Lemma 5.3, $\alpha_{i}=4$ and $\varepsilon_{i}=1, i=1,2,3,4$. In particular, there are no loops in $\Gamma_{2}$. Therefore $\Gamma_{1} \cong \Gamma_{2} \cong G(4,4,4,4)$.

The arrangement of the endpoints of the edges of $\Gamma_{1}$ at the vertices + and - is as shown in Figure 37. Let the edges with label $x$ at vertex + be $A_{1}, A_{2}, \ldots, A_{8}$ as indicated. In $\Gamma_{2}$, these are the edges with label + at $x$ and label - at $y$. Around the vertex $x$, they appear in the order $A_{1}, A_{1+d} A_{1+2 d}, \ldots$, where $d=d_{12}$. Here the two possibilities for $d$ are 1 and 3 .

Consider first the case $d=1$. Then no pair $A_{i}, A_{i+1}$ (subscripts understood modulo 8) can be parallel in $\Gamma_{2}$, either because they are parallel in $\Gamma_{1}$, or because their endpoints at the vertices + and - of $\Gamma_{1}$ violate Lemma 2.5(i). This is a contradiction. Similarly, if $d=3$, one easily checks that no pair $A_{i}, A_{i+3}$ can be parallel in $\Gamma_{2}$ because of Lemma 2.5(i).

$\Delta=7$ is impossible. Here $\sum_{i=1}^{4} \alpha_{i}=14$. From Lemma 5.3 it easily follows that $\Gamma_{1} \cong G(4,4,4,2)$, and $\varepsilon_{i}=1, i=1,2,3,4$. Hence there are no loops in $\Gamma_{2}$, so $\Gamma_{2} \cong G\left(\beta_{1}, \beta_{2}, \beta_{3}, \beta_{4}\right)$, say.

By Lemma 2.1, the edges in any parallelism class in $\Gamma_{1}$ must belong to distinct parallelism classes in $\Gamma_{2}$. Therefore $\left(\beta_{1}, \beta_{2}, \beta_{3}, \beta_{4}\right)$ is some permutation of $(4,4,3,3)$. But this contradicts Lemma 5.3.

$\Delta=6$ is impossible. Here $\sum_{i=1}^{4} \alpha_{i}=12$. It is straightforward to check that the only possibilities for $\Gamma_{1}$ allowed by Lemma 5.3 are: $G(4,4,4,0), G(4,4,2,2)$, $G(4,2,4,2)$, and $G(3,3,3,3)$. Since all $\varepsilon_{i}$ 's are 1 in all cases, there are no loops in $\Gamma_{2}$. So $\Gamma_{2} \cong G\left(\beta_{1}, \beta_{2}, \beta_{3}, \beta_{4}\right)$.

Let $A_{1}, A_{2}, \ldots, A_{6}$ be the edges of $\Gamma_{1}$ with label $x$ at vertex + , numbered in order around that vertex. Since here $d=1$, these edges appear in the same order around vertex $x$ of $\Gamma_{2}$. We consider the four possibilities listed above for $\Gamma_{1}$.

Suppose $\Gamma_{1} \cong G(4,4,4,0)$. See Figure 38 . We then see that no consecutive pair $A_{i}, A_{i+1}$ can be parallel in $\Gamma_{2}$, either because they are parallel in $\Gamma_{1}$ or because of Lemma 2.5(i). This contradicts the form of $\Gamma_{2}$.

Suppose $\Gamma_{1} \cong G(4,4,2,2)$. See Figure 39 . Here, the only consecutive pair $A_{i}, A_{i+1}$ that is permitted to be parallel in $\Gamma_{2}$ by the two restrictions used above is $A_{5}, A_{6}$. But this would lead to too many parallelism classes on $\Gamma_{2}$.

In the other two cases, $G(4,2,4,2)$ and $G(3,3,3,3)$, we argue similarly and easily conclude that no pair $A_{i}, A_{i+1}$ can be parallel in $\Gamma_{2}$.

We now consider the case $n_{2} \geq 3$. Let $P_{1}, P_{2}, P_{3}, P_{4}$ be the parallelism classes of edges in $\Gamma_{1}$, where $P_{i}$ has size $\alpha_{i}$. Let $P_{i}^{+}, P_{i}^{-}$denote the set of labels at the end of $P_{i}$ at vertex,+- respectively. If an edge in $P_{i}$ has label $x$ at vertex + , then it has label $\pi_{i}(x) \equiv x+p_{i}\left(\bmod n_{2}\right)$ at - for some $p_{i}$. Thus we have a 
C. McA. GORDON

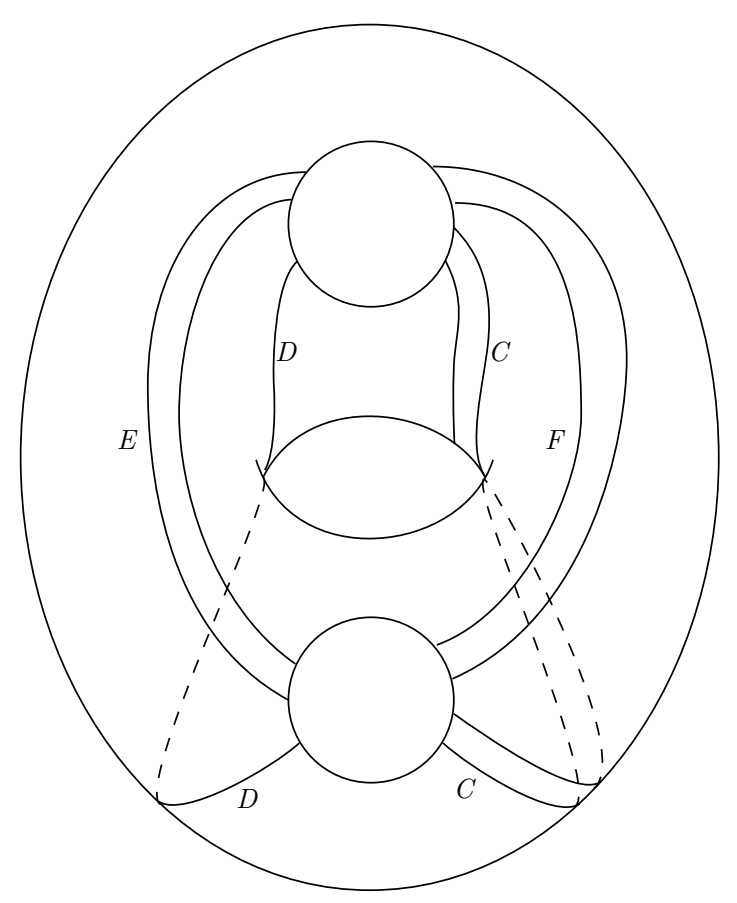

(i)

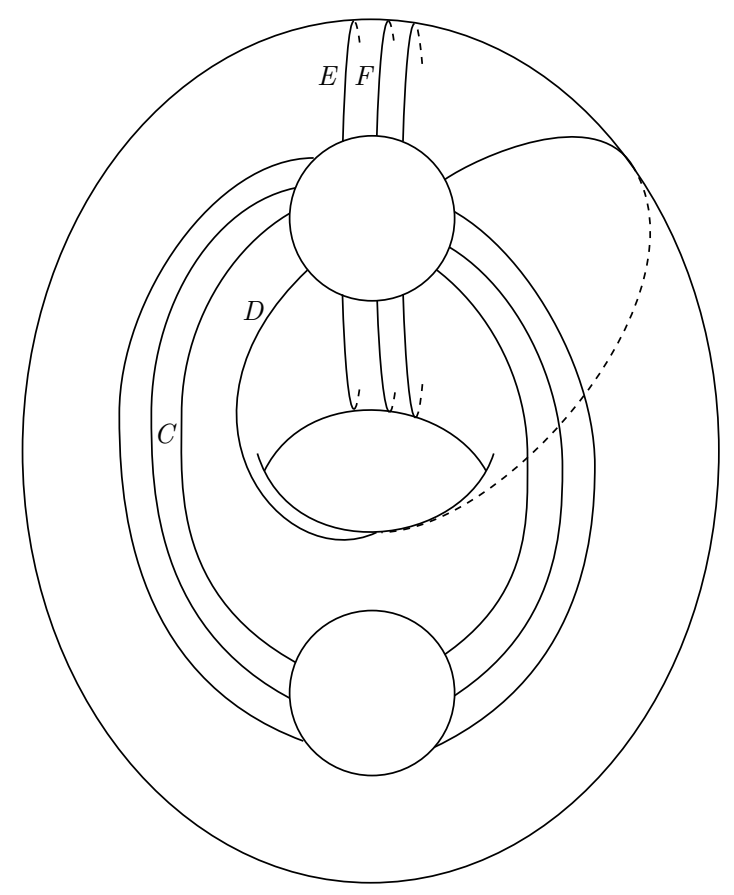

(ii)

Figure 35 


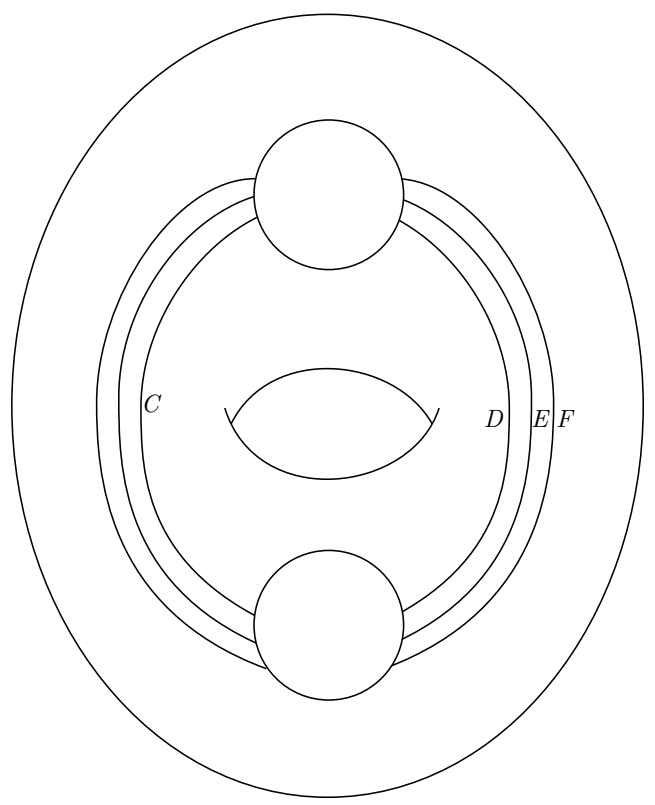

(i)

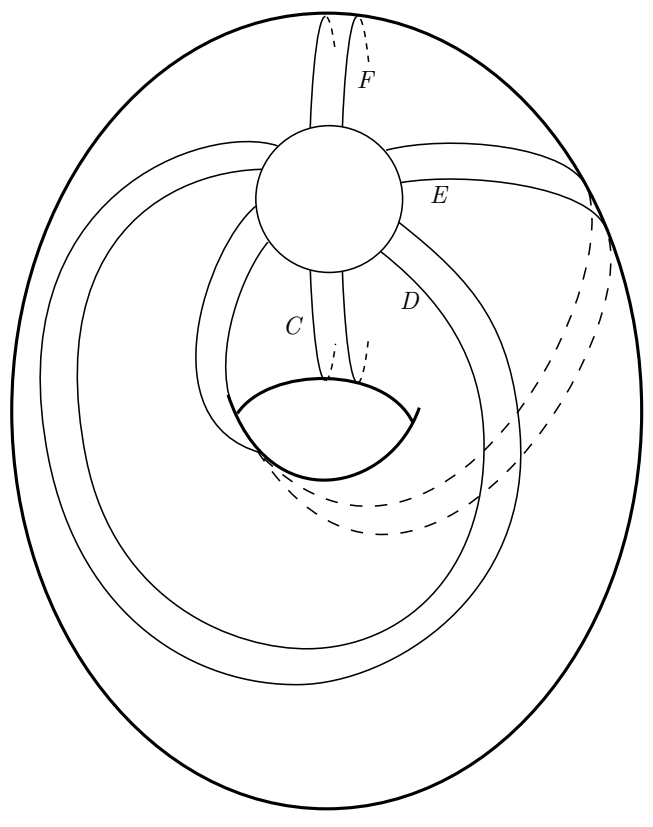

(ii)

FiguRe 36 


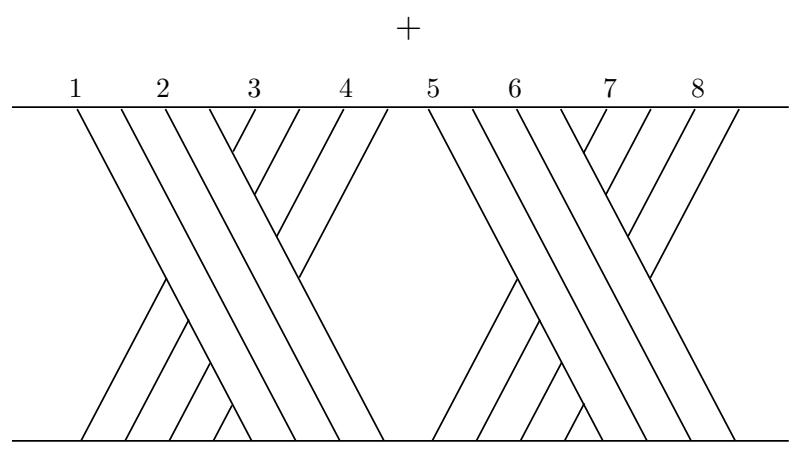

FIGURE 37

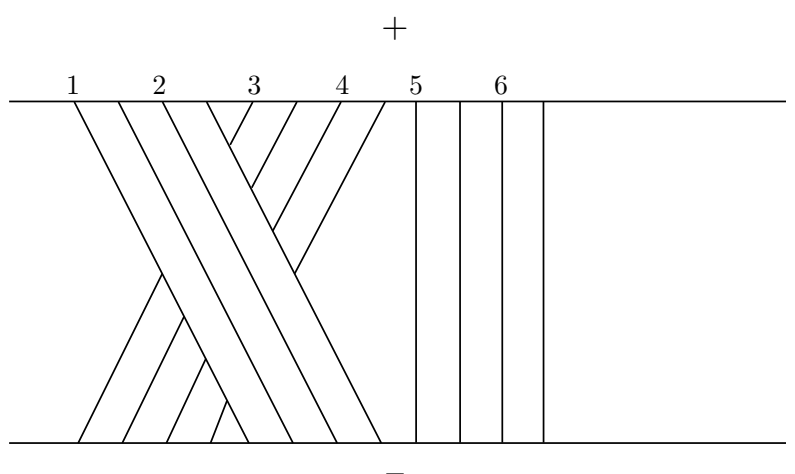

FIGURE 38

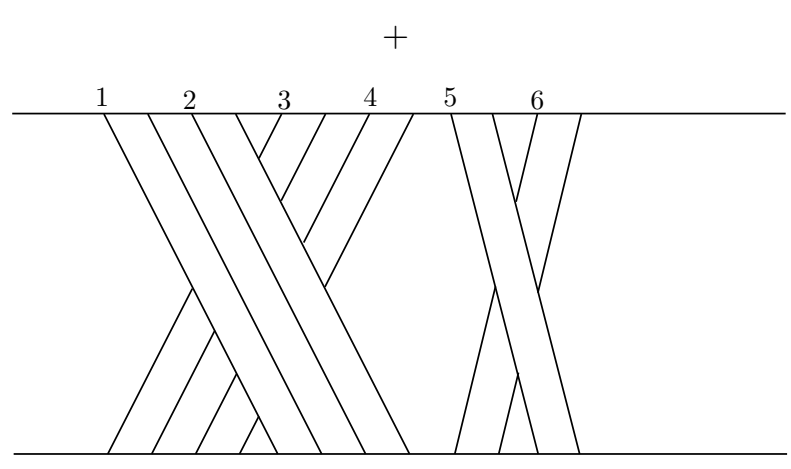

FiguRE 39

well-defined permutation $\pi_{i}$ of $\left\{1,2, \ldots, n_{2}\right\}$, even if $\alpha_{i}<n_{2}$ (but provided that $\left.\alpha_{i} \neq 0\right), i=1,2,3,4$.

Note that since $\sum \alpha_{i}=\Delta n_{2} \geq 6 n_{2}$, some $\alpha_{i} \geq n_{2}+1$, and hence the corresponding $\pi_{i}$ has only one orbit, by Lemma 4.2. 


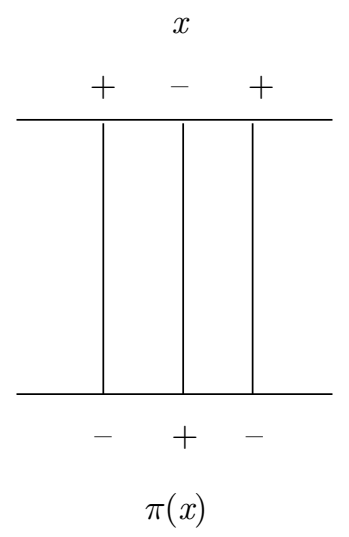

FiguRE 40

Lemma 7.3. The permutations $\pi_{i}, i=1,2,3,4$, are not all equal.

Proof. Suppose $\pi_{i}=\pi, i=1,2,3,4$. Then for any vertex $x$ of $\Gamma_{2}$, there are $\Delta$ edges in $\Gamma_{1}$ with label $x$ at vertex + and label $\pi(x)$ at vertex - . Since $\nu(x, \pi(x)) \leq 5$ by Lemma 5.4(i), some pair of these edges must be parallel in $\Gamma_{2}$. This then gives rise to an edge in $\Gamma_{1}$ with label $x$ at - and label $\pi(x)$ at + (see Figure 40). But this implies that $\pi^{2}$ is the identity, and hence $n_{2}=2$ (since $\pi$ has only one orbit), a contradiction.

We now distinguish the two cases $n_{2}=3$ and $n_{2} \geq 4$.

CASE (3). $n_{2}=3$. In the first two lemmas we use the convention that $\{x, y, z\}=$ $\{1,2,3\}$, the vertices of $\Gamma_{2}$.

Lemma 7.4. (i) If $\Gamma_{2}$ contains a loop at $x$, then $\nu(y, z) \leq 2$;

(ii) If $\nu(x, y) \geq 4$, then $\nu(x, z) \leq 2$.

Proof. (i) Cutting the torus $\widehat{F}_{2}$ along a loop at $x$ gives an annulus containing $y$ and $z$ in its interior. The result is now clear.

(ii) If $\nu(x, y) \geq 2$, cutting $\widehat{F}_{2}$ along two edges of $\bar{\Gamma}_{2}$ joining $x$ and $y$ gives an annulus with $z$ in its interior. If there are two additional edges in $\bar{\Gamma}_{2}$ joining $x$ and $y$, then they must be as shown in Figure 41(i), (ii) or (iii). The fact that $\nu(x, z) \leq 2$ now follows by inspection.

Lemma 7.5. Suppose that $\nu(x, y)=\nu(x, z)=3$. Let $Y_{1}, Y_{2}, Y_{3}$ and $Z_{1}, Z_{2}, Z_{3}$ be the edges of $\bar{\Gamma}_{2}$ joining $x$ to $y$ and $z$ respectively. Then (for suitable choice of subscripts) around the vertex $x$ these edges appear in the order $Y_{1}, Z_{1}, Y_{2}, Z_{2}, Y_{3}, Z_{3}$.

Proof. Consider three consecutive parallel edges of $\Gamma_{1}$ in some family of at least four parallel edges. Cutting the torus $\widehat{F}_{2}$ along the corresponding edges of $\bar{\Gamma}_{2}$ gives an annulus $N$.

Since $\nu(x, y)=\nu(x, z)=3$ by hypothesis, there are additional edges $Y, Y^{\prime}$ and $Z, Z^{\prime}$ of $\bar{\Gamma}_{2}$ joining $x$ to $y$ and $z$ respectively. Such an edge is of either type I or type II (see the proof of Lemma 5.4).

If both $Y$ and $Y^{\prime}$ are of type I, then $\nu(x, z)=1$ (see Figure 42). 


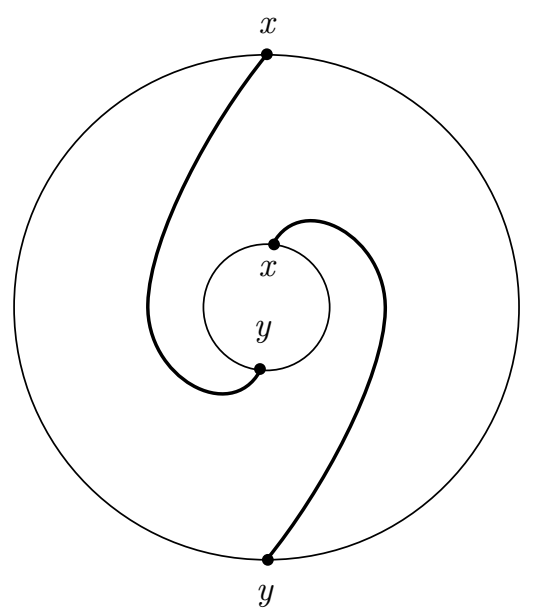

(i)

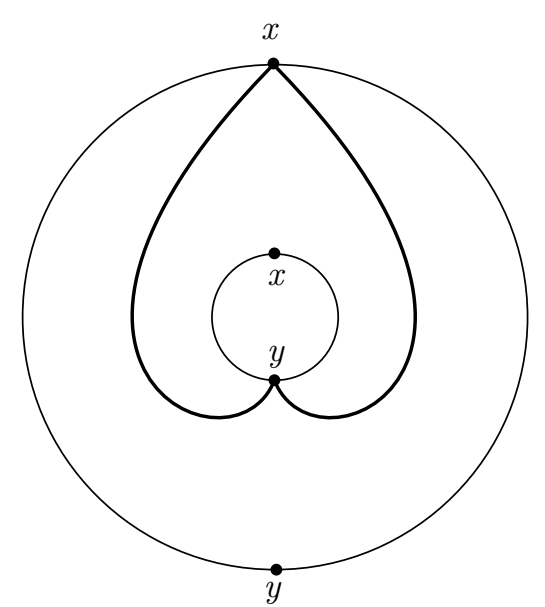

(ii)

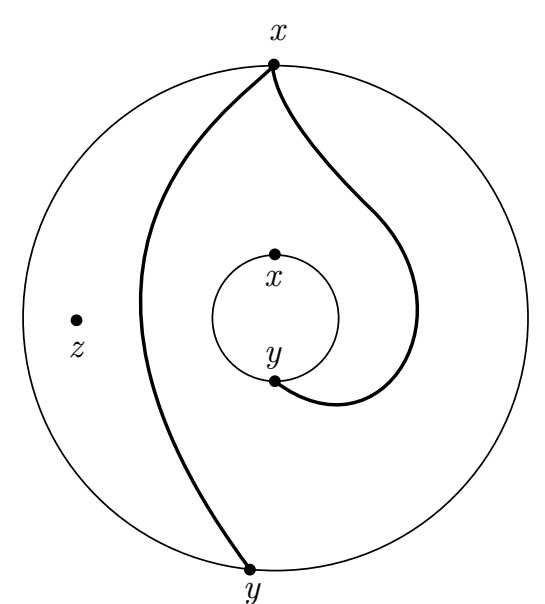

(iii)

FigURE 41

If $Y$ is of type I and $Y^{\prime}$ of type II, then we must have the situation shown in Figure 43.

If both $Y$ and $Y^{\prime}$ are of type II, then we must have the situation shown in either Figure 44(i) or Figure 44(ii).

The result follows by inspection.

Let $\sigma$ denote the permutation (123). Then $\pi_{i}=\sigma, \sigma^{-1}$, or $i d$.

Lemma 7.6. (i) If $\pi_{i}=i d$ then $\alpha_{i} \leq 3$.

(ii) $\alpha_{i} \leq 9, i=1,2,3,4$.

Proof. (i) follows from Lemma 4.2 . 


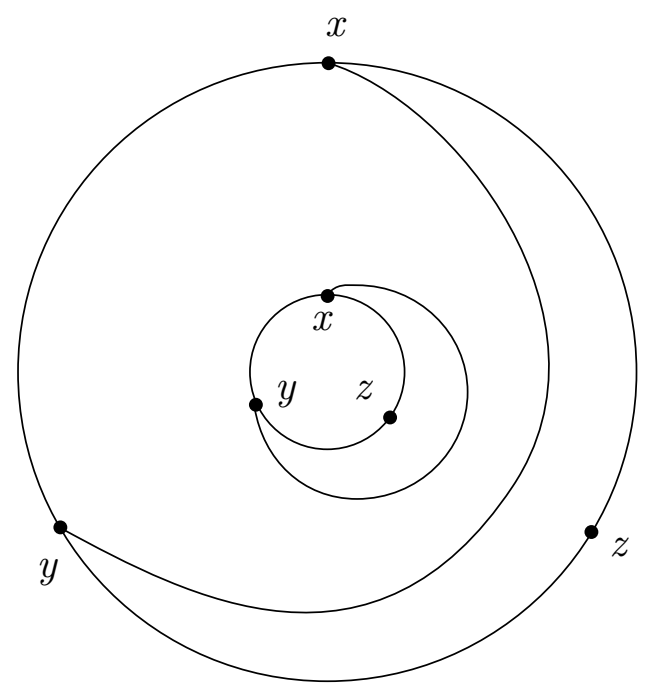

FiguRe 42

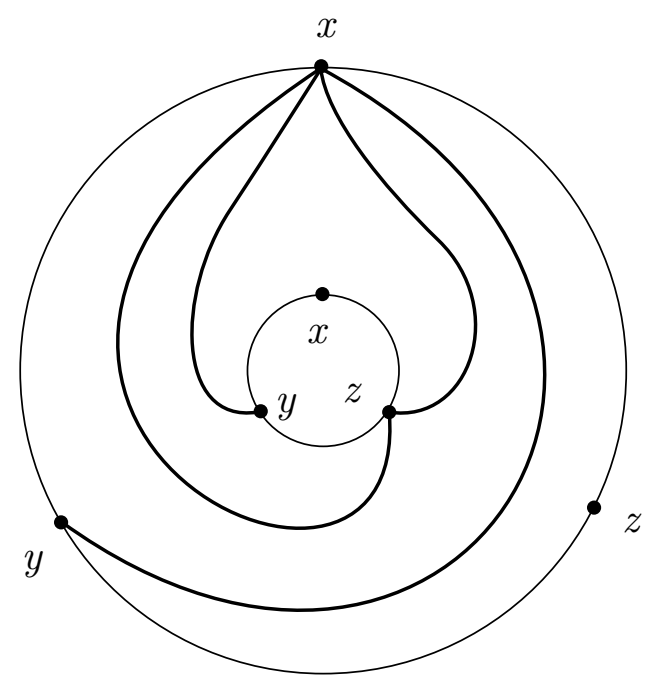

FIGURE 43

(ii) Suppose $\pi_{i} \neq i d$ but $\alpha_{i}>9$. Then for some $x, y$ we have $\nu(x, y) \geq 4$ and $\nu(x, z) \geq 3$ (and $\nu(y, z) \geq 3$ ), by Lemma 2.1. This contradicts Lemma 7.4(ii).

As a preliminary restriction on the possibilities for $\left(\alpha_{1}, \alpha_{2}, \alpha_{3}, \alpha_{4}\right)$ we consider the residue classes of the $\alpha_{i}$ 's modulo 3. Without loss of generality, we assume that 1 is the first label in $P_{1}^{+}$as we go round the vertex + anticlockwise.

Lemma 7.7. The only possibilities (up to cyclic permutation and reversal) for $\left(\alpha_{1}, \alpha_{2}, \alpha_{3}, \alpha_{4}\right)$ and the corresponding permutations $\left(\pi_{1}, \pi_{2}, \pi_{3}, \pi_{4}\right)$ are:

(a) $\left(\alpha_{1}, \alpha_{2}, \alpha_{3}, \alpha_{4}\right) \equiv(1,2,0,0)(\bmod 3),\left(\pi_{1}, \pi_{2}, \pi_{3}, \pi_{4}\right)=\left(\sigma, \sigma, \sigma^{-1}, \sigma^{-1}\right)$;

(b) $\left(\alpha_{1}, \alpha_{2}, \alpha_{3}, \alpha_{4}\right) \equiv(1,1,2,2)(\bmod 3),\left(\pi_{1}, \pi_{2}, \pi_{3}, \pi_{4}\right)=\left(\sigma, \sigma^{-1}, \sigma^{-1}, \sigma\right)$; 


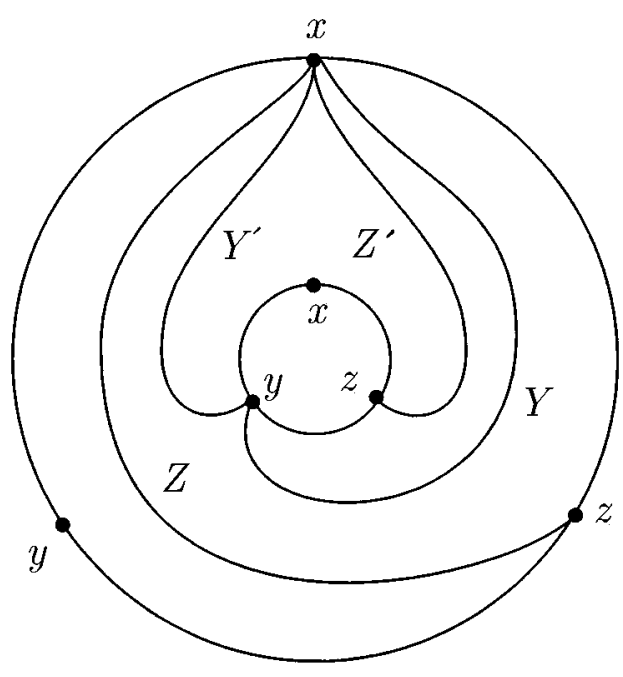

(i)

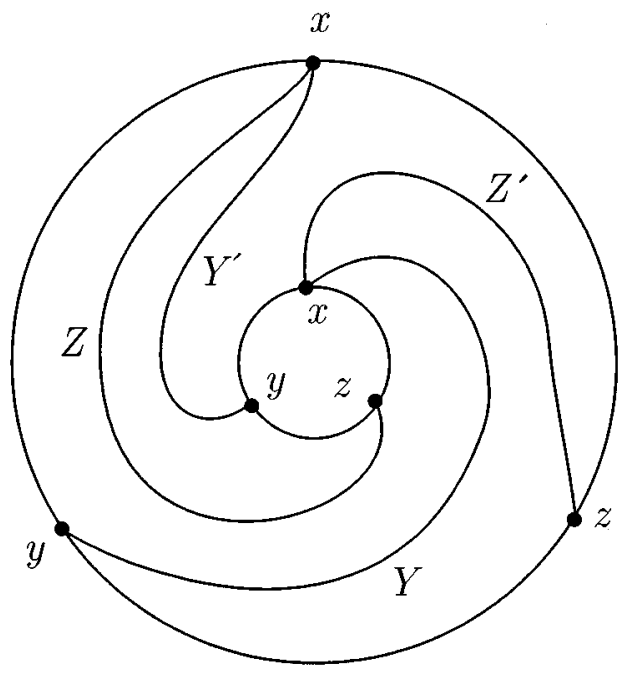

(ii)

FiguRe 44

(c) $\left(\alpha_{1}, \alpha_{2}, \alpha_{3}, \alpha_{4}\right) \equiv(1,0,2,0)(\bmod 3),\left(\pi_{1}, \pi_{2}, \pi_{3}, \pi_{4}\right)=\left(\sigma, i d, \sigma, \sigma^{-1}\right)$.

Proof. Since $\sum \alpha_{i}=3 \Delta$, the only possibilities for $\left(\alpha_{1}, \alpha_{2}, \alpha_{3}, \alpha_{4}\right)$ modulo 3 are: $(0,0,0,0),(1,1,1,0),(2,2,2,0),(1,2,0,0),(1,0,2,0),(1,1,2,2)$ and $(1,2,1,2)$. We examine each of these in turn. Note also that $\sum \alpha_{i} \geq 18$.

$(0,0,0,0)$. Here $\pi_{1}=\pi_{2}=\pi_{3}=\pi_{4}$, contradicting Lemma 7.3.

$\underline{(1,1,1,0)}$. Going around the vertex - clockwise, the first label in $P_{2}^{-}$is either 1 , 2 , or 3. This gives three possibilities for the labeling at - modulo 3 , illustrated in 
(1)

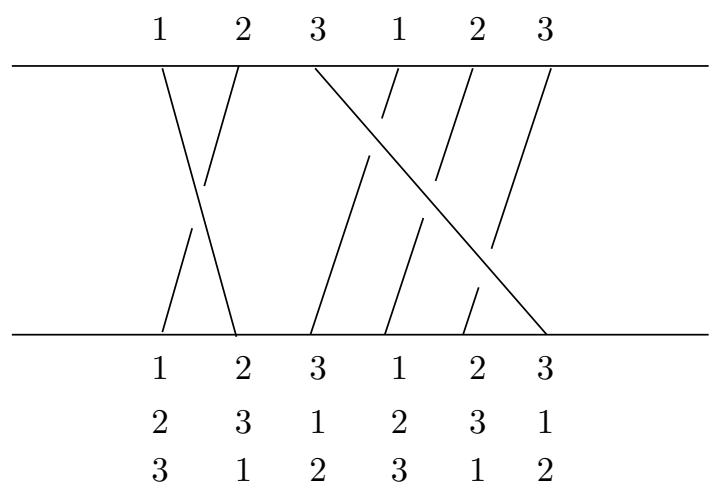

Figure 45

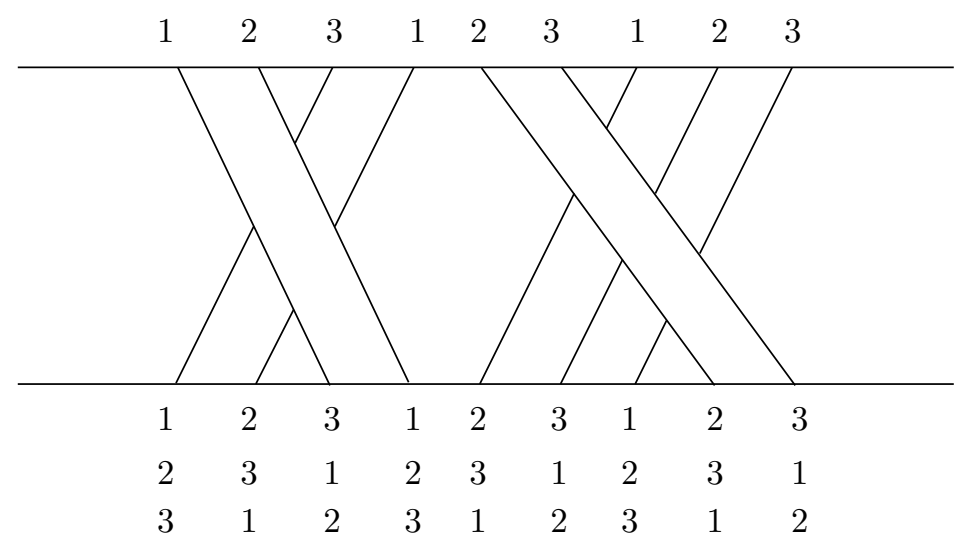

FiguRE 46

Figure 45 (1), (2), and (3) ( $\alpha_{4}$ may be 0 ). (Here, and subsequently, we make the convention that the vertex + is at the top.)

(1) By Lemma 7.6(i), $\alpha_{3}=1$. Also $\nu(1,2) \leq 2$ by Lemma 7.4(i). Therefore, by Lemma 2.1, there are at most two edges in $P_{i}$ with label 1 at one endpoint and label 2 at the other, $i=1,2,4$. Thus $\alpha_{1}, \alpha_{2} \leq 6$ and $\alpha_{4} \leq 7$. By considering the residues of $\alpha_{1}, \alpha_{2}$, and $\alpha_{4}$ modulo 3 this gives $\alpha_{1}, \alpha_{2} \leq 4$ and $\alpha_{4} \leq 6$. Hence $\sum \alpha_{i} \leq 4+4+1+7=15$, a contradiction.

(2) Here $\alpha_{2}=1$ by Lemma 7.6(i), and $\nu(1,3) \leq 2$ by Lemma 7.4(i). Therefore $\alpha_{1}, \alpha_{3} \leq 6$ (and hence $\leq 4$ ). Also $\alpha_{4} \leq 3$ by Lemma 7.6(i). Hence $\sum \alpha_{i} \leq 12$, a contradiction.

(3) This case is isomorphic to case (1) above.

$\underline{(2,2,2,0)}$. Again we have three possible labelings modulo 3 at the vertex -, illustrated in Figure 46 (1), (2) and (3).

(1) Here $\alpha_{3}=2$, and $\nu(1,3) \leq 2, \nu(1,2) \leq 2$. Hence $\alpha_{1}, \alpha_{2} \leq 5$ and $\alpha_{4} \leq 6$. Since $\sum \alpha_{i} \geq 18$, the only possibility is $\left(\alpha_{1}, \alpha_{2}, \alpha_{3}, \alpha_{4}\right)=(5,5,2,6)$. Let $A, B, C$ be the edges of $\Gamma_{1}$ shown in Figure 47. By Lemma 2.1, $A$ and $B$ are not parallel in $\Gamma_{2}$, and hence, since $\nu(1,3) \leq 2, C$ must be parallel in $\Gamma_{2}$ to either $A$ or $B$. But this is impossible by Lemma $2.5(\mathrm{i})$. 


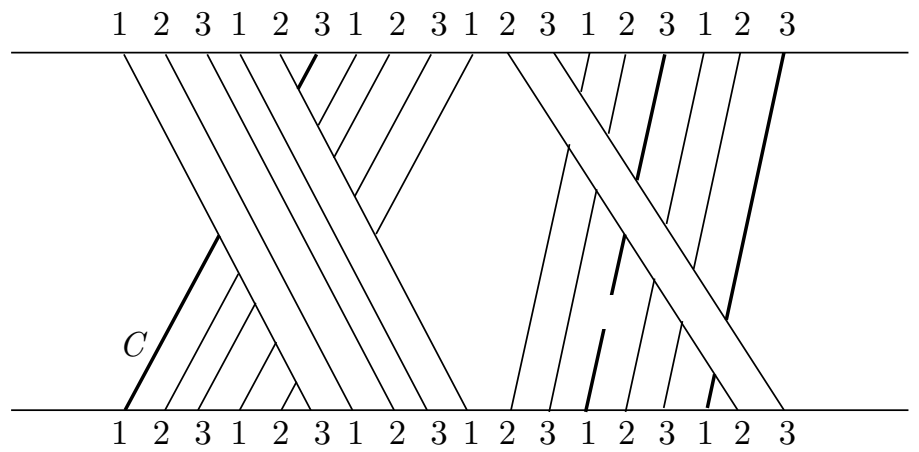

FigURE 47

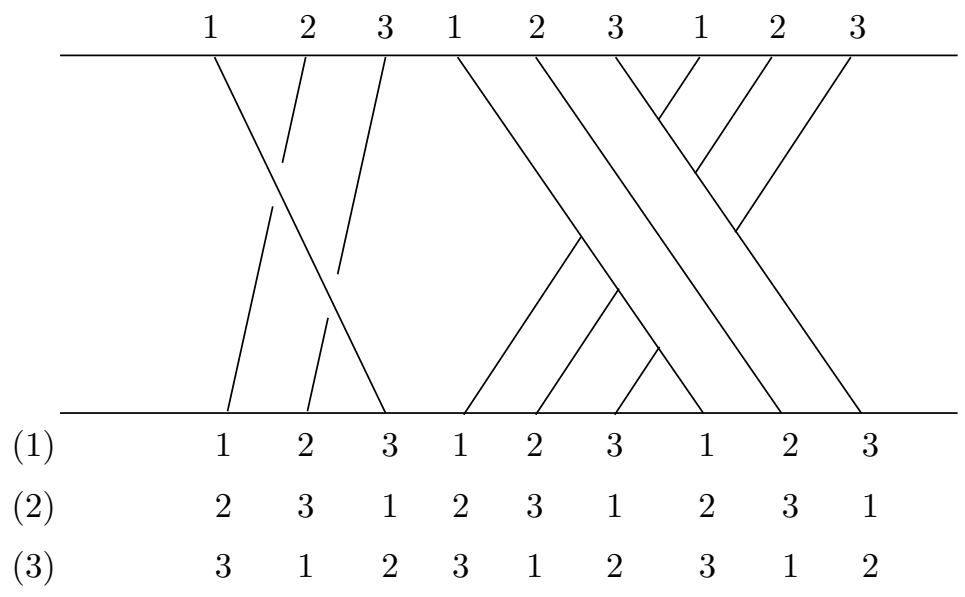

Figure 48

(2) This case in isomorphic to (1).

(3) Here $\alpha_{3}=2$. Hence $\alpha_{1}, \alpha_{3} \leq 6$, and so $\leq 5$. Also $\alpha_{4} \leq 3$. Therefore $\sum \alpha_{i} \leq 15$, a contradiction.

$(1,2,0,0)$. The three possible labelings modulo 3 at the vertex - are illustrated in Figure 48 (1), (2), and (3).

(1) Here $\alpha_{3}$ and $\alpha_{4}$ are either 0 or 3 . If both are 0 , then $\alpha_{1}+\alpha_{2} \geq 18$, and hence $\alpha_{1}$ or $\alpha_{2}>9$, contradicting Lemma 7.6(ii). If either $\alpha_{3}$ or $\alpha_{4}$ is 3 , then $\nu(x, y) \leq 2$ for all $x, y$, giving $\alpha_{1} \leq 4$ and $\alpha_{2} \leq 5$. Hence $\sum \alpha_{i} \leq 15$.

(2) Here $\alpha_{1}=1$ and $\alpha_{2}=2$, and $\nu(x, y) \leq 2$ for all $x, y$. Hence $\alpha_{3}, \alpha_{4} \leq 6$. Therefore $\sum \alpha_{i} \leq 15$.

(3) is possibility (a) of the lemma.

$(1,0,2,0)$. The three possible labelings modulo 3 at the vertex - are illustrated in Figure 49 (1), (2), and (3).

(1) Here $\alpha_{1}=1, \alpha_{3}=2$, and hence $\alpha_{2}, \alpha_{4} \leq 6$, giving $\sum \alpha_{i} \leq 15$.

(2) This is possibility (c) of the lemma.

(3) is isomorphic to (2). 


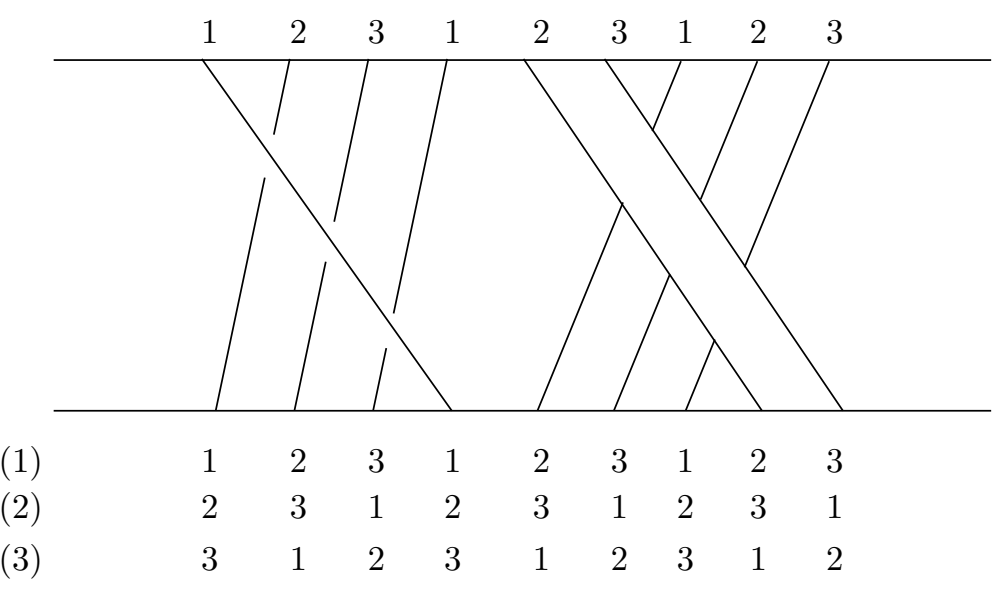

FIGURE 49

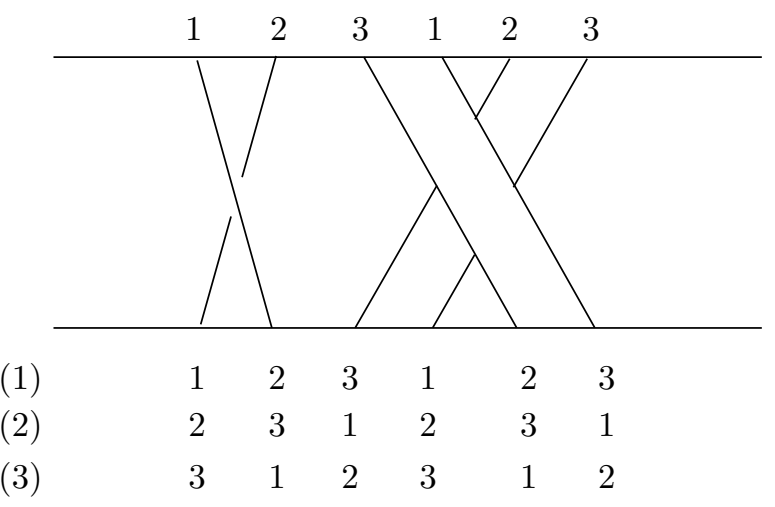

FiguRE 50

$(1,1,2,2)$. The three possible labelings at - are illustrated in Figure 50 (1), (2), and (3).

(1) is possibility (b) of the lemma.

(2) and (3) are ruled out by arguments similar to those given above.

$(1,2,1,2)$. Here $\pi_{1}=\pi_{2}=\pi_{3}=\pi_{4}$, contradicting Lemma 7.3.

We now proceed to rule out the possibilities left after Lemma 7.7. Since $\alpha_{1} \leq 9$ by Lemma 7.6(ii), there are only a finite number of cases to consider.

Lemma 7.8. Case (a) of Lemma 7.7 is impossible.

Proof. Since $\alpha_{i} \leq 9$, we have that $\alpha_{1}=1,4$, or $7, \alpha_{2}=2,5$, or 8 , and $\alpha_{3}, \alpha_{4}=$ $0,3,6$, or 9 .

As an example, suppose that $\left(\alpha_{1}, \alpha_{2}, \alpha_{3}, \alpha_{4}\right)=(*, *, 6,9)$. Then the edges of $\Gamma_{1}$ in $P_{3} \cup P_{4}$ are as in Figure 51. Since $P_{4}$ contains three edges with label 1 at + and 3 at,$- \nu(1,3) \geq 3$ by Lemma 2.1. Now let $A, B, C$ be the three edges in $P_{4}$ with label 2 at + and 1 at -, and let $X$ be one of the edges in $P_{3}$ with label 2 at + and 1 at -. Let $A^{ \pm}$denote the endpoints of $A$ at \pm , and similarly for $B, C$, and $X$. One 


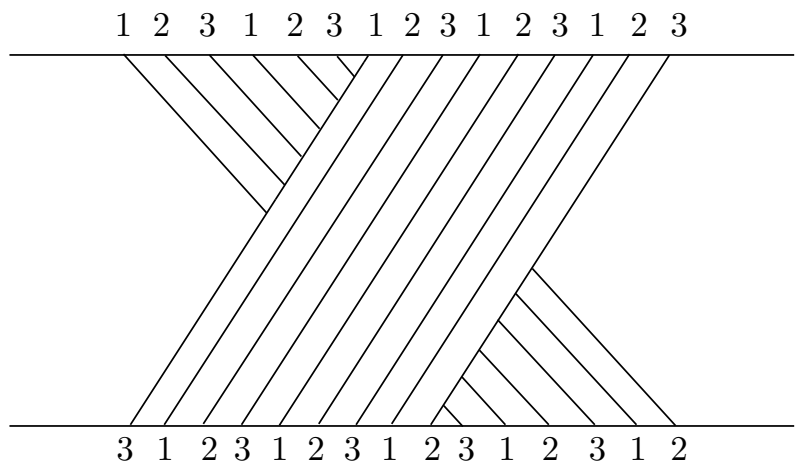

FIGURE 51

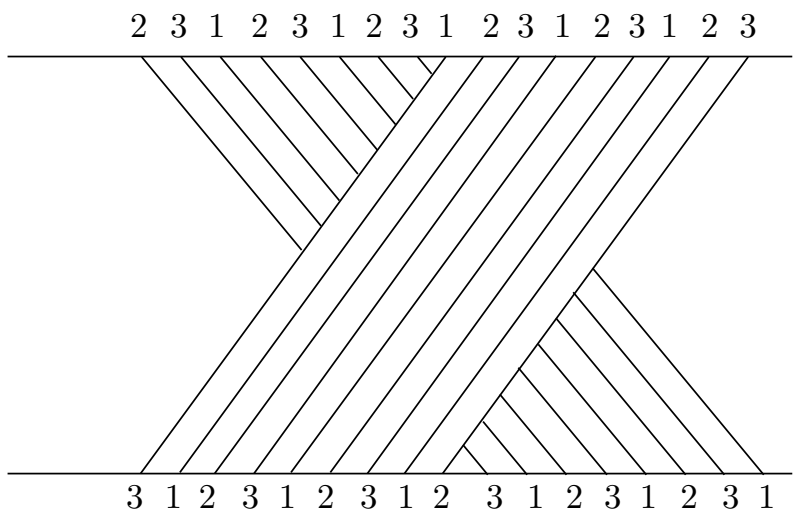

FiguRe 52

readily checks that $\delta_{1}\left(X^{+}, Y^{+}\right) \neq \delta_{1}\left(X^{-}, Y^{-}\right)$for $Y=A, B$, or $C$, and hence $X$ is not parallel in $\Gamma_{2}$ to either $A, B$, or $C$, by Lemma 2.5(i). Since $A, B$ and $C$ are pairwise non-parallel in $\Gamma_{2}$ by Lemma 2.1 , we get $\nu(1,2) \geq 4$. But this contradicts Lemma 7.4(ii).

The case $(*, *, 9,6)$ is ruled out in exactly the same way, and a similar argument rules out $(7,8, *, *)$.

Now consider the case $(*, 8,9, *)$, illustrated in Figure 52. Again, considering $P_{3}$ shows that $\nu(x, y) \geq 3$ for all $x, y$, by Lemma 2.1. Let $A, B, C$ be the edges in $P_{3}$ with label 1 at + and 3 at - , and let $X$ be one of the edges in $P_{2}$ with label 3 at + and 1 at - . One readily verifies, this time using Lemma 2.5(ii), that $X$ is not parallel in $\Gamma_{2}$ to either $A, B$, or $C$. Hence $\nu(1,3) \geq 4$, contradicting Lemma 7.4(ii) as before.

Similar arguments rule out the cases $(*, 2,9, *),(*, 8,3, *)$, and $(4, *, *, 9)$.

Next consider the case $(*, 5,6, *)$, shown in Figure 53. By considering the four edges in $P_{2} \cup P_{3}$ with label 2 at one end and 3 at the other, and using Lemmas 2.1 and 2.5 (iii), we see that $\nu(2,3) \geq 4$. Similarly, $\nu(3,1) \geq 4$. But this contradicts Lemma 7.4(ii).

A similar argument rules out $(7, *, *, 6)$. 


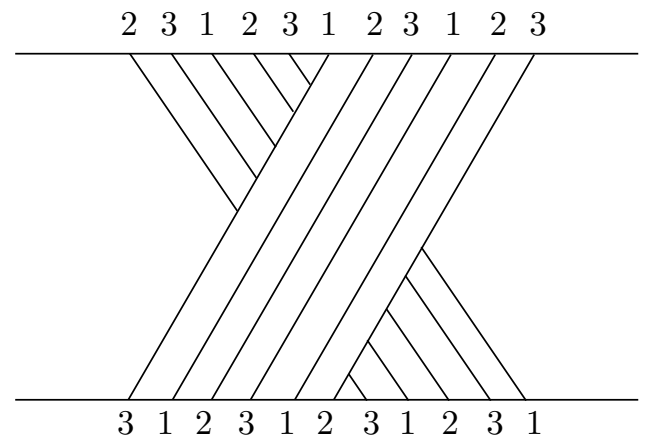

FIGURE 53

Since $\alpha_{3} \equiv 0(\bmod 3)$, the argument used to rule out $(*, 8,9, *)$ applies essentially verbatim to rule out $(*, 8, *, 9)$; one just replaces $P_{3}$ by $P_{4}$. In the same way, the arguments in the next five cases also rule out, respectively, $(*, 2, *, 9),(*, 8, *, 3)$, $(4, *, 9, *),(*, 5, *, 6)$, and $(7, *, 6, *)$,

To eliminate some more possibilities, we use a different line of argument. Since $\Gamma_{1}$ has at most four parallelism classes of edges, the number of edges in any parallelism class in $\Gamma_{2}$ is at most four, by Lemma 2.1. Hence if two parallel edges in $\Gamma_{2}$ have label + (say) at a vertex $x$, then their endpoints at $x$ are adjacent among all edge endpoints with label + at $x$. Therefore the endpoints at the vertex + of the corresponding edges in $\Gamma_{1}$, which have label $x$, are " $d$ apart" among all edge endpoints with label $x$ at + (where $d=d_{21}$ ), i.e., are separated by $d-1$ such endpoints.

To see how this may be used, consider the case $(4,8, *, *)$, illustrated in Figure 54 . Since $P_{2}$ contains three edges with endpoint labels 1 and $3, \nu(1,3) \geq 3$. Therefore $\nu(2,3) \leq 3$ by Lemma 7.4(ii). Let $A, B, C$ be the edges in $P_{2}$ with label 2 at + and 3 at - . (We adopt the convention that if we label edges in a given $P_{i}$ alphabetically, then the alphabetic ordering agrees with the anticlockwise ordering around the vertex +; see edges $A$ and $B$ in Figure 47.) Let $X$ be the edge in $P_{1}$ with label 2 at + and 3 at - . Since $\nu(2,3) \leq 3, X$ must be parallel in $\Gamma_{2}$ to either $A, B$, or $C$. By Lemma 2.5(i), $X$ must be parallel to $B$. Therefore $d=2$, and hence $\Delta$ is odd. This rules out $(4,8,6,0)(=(4,8,0,6))$, and $(4,8,6,6)$, which have $\Delta=6$ and 8 respectively.

Similarly, one shows that for $(7,5, *, *),(*, *, 6,6),(*, *, 9,3)$, and $(*, *, 3,9)$, we must have $d=2$, and hence $\Delta$ odd. This rules out $(1,5,9,3),(1,5,3,9),(4,2,6,6)$, $(7,5,3,3),(7,5,3,9),(7,5,9,3)$, and $(7,5,9,9)$.

The only cases left are $(1,5,9,9),(7,5,0,9)$, and $(1,8,6,6)$.

Consider $(1,5,9,9)$. The argument just given (applied to $P_{3}$ and $\left.P_{4}\right)$ shows that for $(*, *, 9,9)$ we must have $d=3$. Now let $A, B, C$ be the edges in $P_{3}$ with label 2 at + and label 1 at - , and let $X, Y$ be the edges in $P_{1}, P_{2}$ respectively with label 1 at + and label 2 at - . See Figure 55 . Since $\nu(1,2) \leq 3, X$ must be parallel in $\Gamma_{2}$ to $A, B$, or $C$, and similarly for $Y$. Using Lemma 2.5(ii), we see that $X$ and $Y$ must be parallel to $B$, and hence to each other. But this implies $d=1$.

Finally, the cases $(7,5,0,9)$ and $(1,8,6,6)$ will be eliminated using Lemma 7.5 .

Consider $(7,5,0,9)$. Let $A, B, C$ be the edges in $P_{4}$ with label 1 at + and 3 at - . Let $X, Y$ and $U, V$ be the edges in $P_{1}$ and $P_{2}$ respectively with label 3 at + and 1 


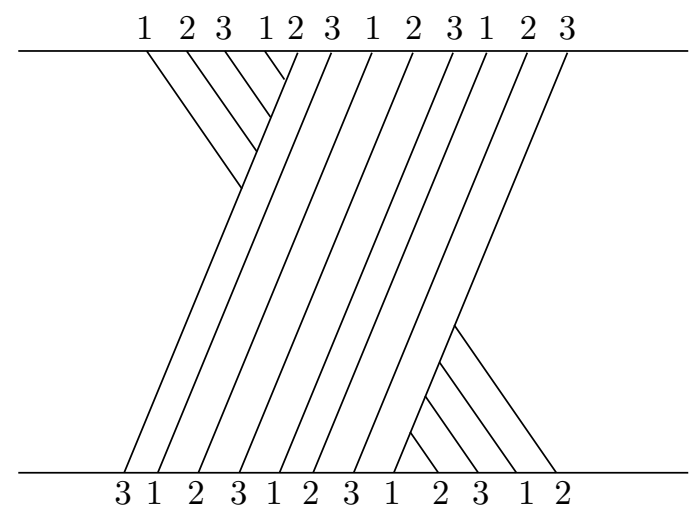

FiguRE 54

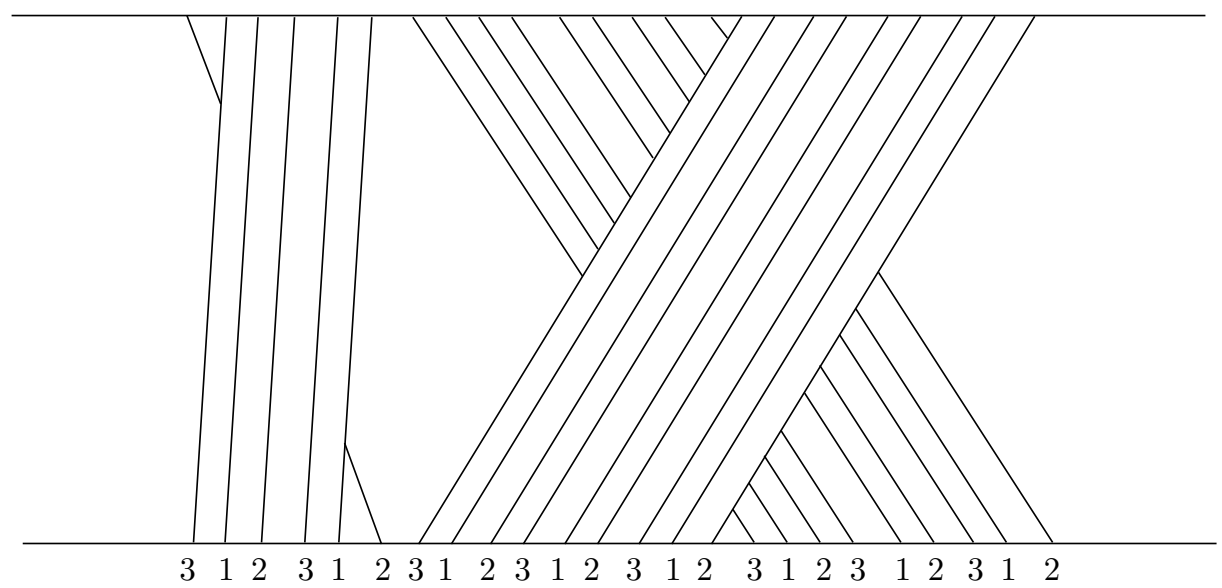

FIGURE 55

at - (see Figure 57). Using Lemmas 2.1 and 2.5(ii), and the fact that $\nu(1,3) \leq 3$, we see that these seven edges fall into exactly the following parallelism classes in $\Gamma_{2}:\{A\},\{B, X, U\},\{C, Y, V\}$. Since $X$ is parallel to $U$, we have $d=2$. Hence $A$ and $C$ are adjacent at vertex 1 among edges of $\Gamma_{2}$ with label + at 1 . The two parallelism classes $\{A\}$ and $\{C, Y, V\}$ then give rise to two edges of $\bar{\Gamma}_{2}$ that join 1 to 3 and are adjacent at 1 . Since $\nu(1,3)=\nu(1,2)=3$, this contradicts Lemma 7.5.

$(1,8,6,6)$ can be handled similarly, by considering the three edges in $P_{2}$ with label 3 at,+ 1 at - , and the four edges in $P_{3} \cup P_{4}$ with label 1 at,+ 3 at - . See Figure 56.

Lemma 7.9. Case (b) of Lemma 7.7 is impossible.

Proof. Here $\alpha_{1}, \alpha_{2}=1,4$, or 7 , and $\alpha_{3}, \alpha_{4}=2,5$, or 8 .

Exactly as in the proof of the previous lemma, $(*, 7,8, *),(7, *, *, 8),(*, *, 5,8)$, and $(*, *, 8,5)$ are impossible, and in a similar way one rules out $(4,7, *, *)$ and $(7,4, *, *)$. 


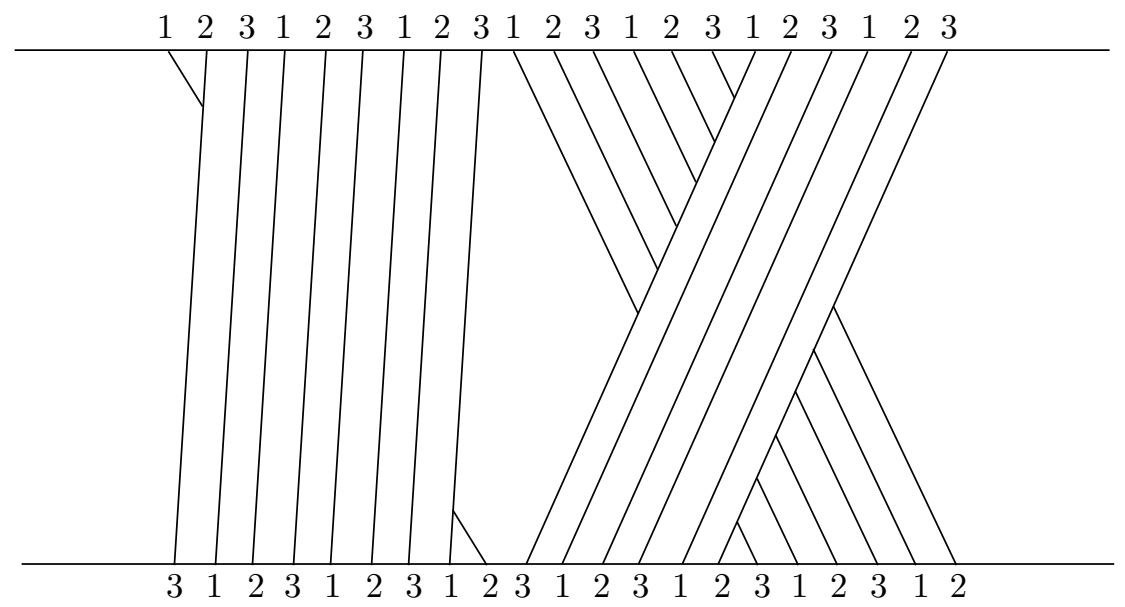

FiguRe 56

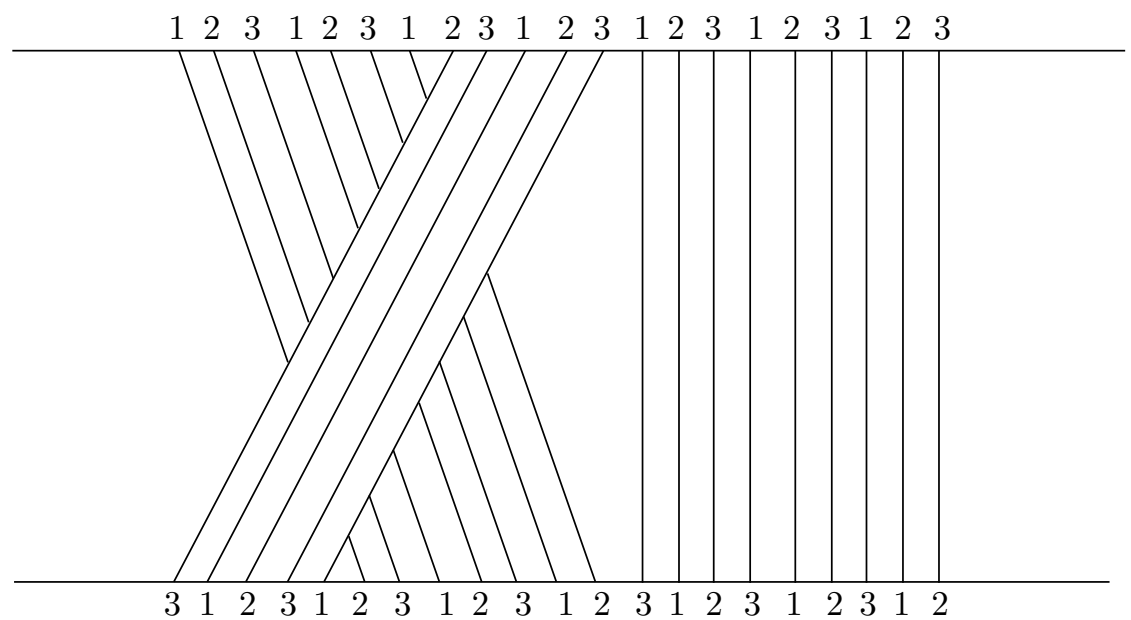

FiguRE 57

Also, $(*, 7, *, 8)$ is ruled out by considering the three edges $A, B, C$ in $P_{4}$ with label 2 at + and 3 at - , and an edge $X$ in $P_{2}$ with label 3 at + and 2 at - . See Figure 58. One checks that by Lemmas 2.1 and 2.5(ii), $X, A, B, C$ are pairwise non-parallel in $\Gamma_{2}$, contradicting $\nu(2,3) \leq 3$. Similarly, $(7, *, 8, *)$ is impossible.

Again as in the proof of the previous lemma, in the cases $(*, 7,5, *),(7, *, *, 5)$, $(*, 4,8, *)$, and $(4, *, *, 8)$ we must have $d=2$, and so $\Delta$ is odd.

It is straightforward to check that the only cases left after these exclusions are $(1,1,8,8),(4,4,5,5),(1,4,8,8),(7,7,2,2)$, and $(7,7,5,2)$.

First consider $(1,1,8,8)$. Let $A, B, C$ be the edges in $P_{3}$ with label 3 at + and 2 at - , and $X, Y, Z$ the edges in $P_{4}$ with label 2 at + and 3 at - (see Figure 59). By arguments that are by now familiar, these edges fall into the parallelism classes $\{A, X\},\{B, Y\}$, and $\{C, Z\}$ in $\Gamma_{2}$. Here $\Delta=6$, and so $d=1$. Therefore $A$ and $B$, and $B$ and $C$, are adjacent at vertex 3 of $\Gamma_{2}$ among edges with label + at 3 . 


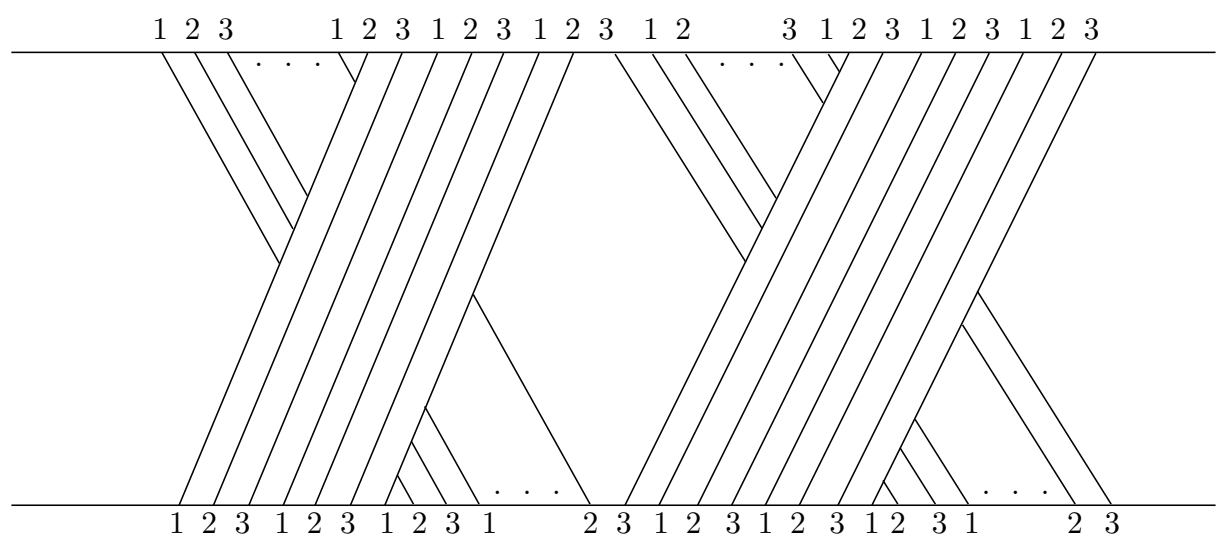

FiguRE 58

$\begin{array}{lllllllllllllllllll}1 & 2 & 3 & 1 & 2 & 3 & 1 & 2 & 3 & 1 & 2 & 3 & 1 & 2 & 3 & 1 & 2 & 3\end{array}$

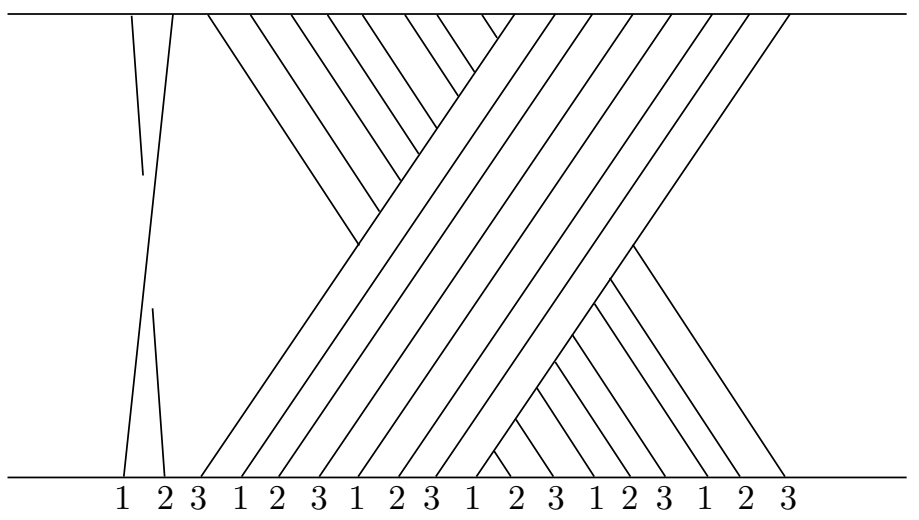

FIGURE 59

Similarly $X$ and $Y$, and $Y$ and $Z$, are adjacent at 3 among edges with label - at 3. Thus $\{A, X\},\{B, Y\}$, and $\{C, Z\}$ give rise to three edges in $\bar{\Gamma}_{2}$ joining vertices 3 and 2 that are adjacent at 3 . This contradicts Lemma 7.5.

The case $(1,4,8,8)$ is handled similarly, using the fact that here $d=2$. Specifically, if $A, B, C, X, Y, Z$ are the edges described above, then $A$ and $C$, and $X$ and $Z$, are adjacent at vertex 3 among edges with label + (resp. -) at 3 . Therefore $\{A, X\}$ and $\{C, Z\}$ give rise to edges in $\bar{\Gamma}_{2}$ joining 3 to 2 that are adjacent at 3 , again contradicting Lemma 7.5.

The remaining cases, $(4,4,5,5),(7,7,2,2)$, and $(7,7,5,2)$, are ruled out by arguments analogous to those just given, using Lemma 7.5. Note that $d=1$ in the first two cases and $d=2$ in the third. We omit the details.

Lemma 7.10. Case (c) of Lemma 7.7 is impossible.

Proof. Here $\alpha_{2}=0$ or 3 .

If $\alpha_{2}=3$ then $\nu(x, y) \leq 2$ for all $x, y$, and therefore $\alpha_{1} \leq 4, \alpha_{3} \leq 5$ and $\alpha_{4} \leq 6$. Hence $\left(\alpha_{1}, \alpha_{2}, \alpha_{3}, \alpha_{4}\right)=(4,3,5,6)$. But $(*, *, 5,6)$ was ruled out in the proof of Lemma 7.8. 


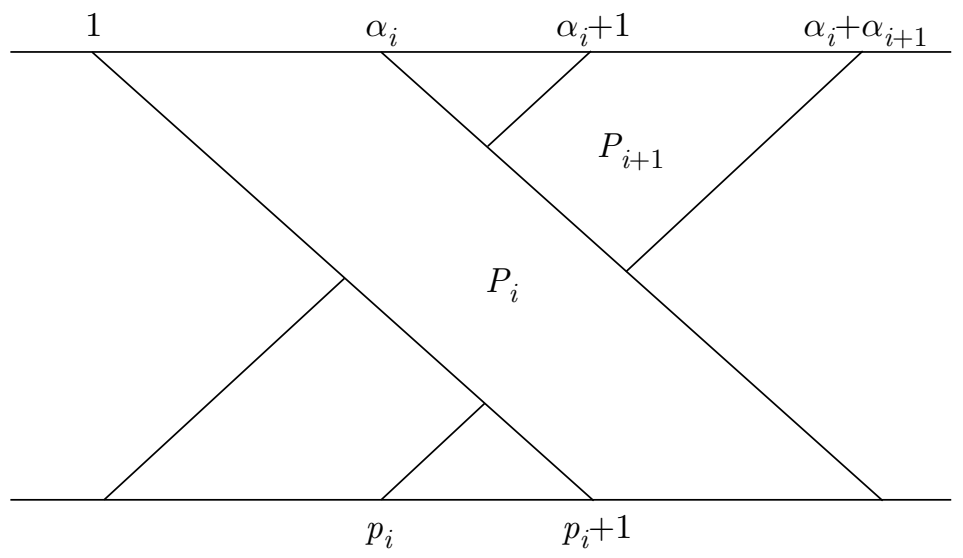

FiguRE 60

So suppose $\alpha_{2}=0$. The cases $(7,0,8, *),(*, 0,8,9),(*, 0,5,6),(*, 0,2,9)$, and $(4,0, *, 9)$ were ruled out in the proof of Lemma 7.8. Since $\alpha_{1}=1,4$, or $7, \alpha_{3}=2,5$ or 8 , and $\alpha_{4}=3,6$, or 9 , it is easy to check that this leaves only $(4,0,8,6)$ and $(7,0,5,9)$. But these were also ruled out in the proof of Lemma 7.8.

CASE (4). $n_{2} \geq 4$. Write $n_{2}=n$.

Lemma 7.11. $\alpha_{i} \leq 2 n, i=1,2,3,4$.

Proof. This follows from Corollary 5.5.

Corollary 7.12. $\Delta \leq 7$.

Proof. $\sum \alpha_{i}=\Delta n$, and hence $\Delta \leq 8$ by Lemma 7.11. Also, if $\Delta=8$ then $\alpha_{i}=2 n$, $i=1,2,3,4$, and therefore $\pi_{1}=\pi_{2}=\pi_{3}=\pi_{4}$, contradicting Lemma 7.3.

Our next goal is to prove Lemma 7.15. First we prove the following (essentially weaker) lemma, which will help us to eliminate certain small cases in Lemma 7.15.

Lemma 7.13. If $\alpha_{i}+\alpha_{j}>3 n$ then $\pi_{i}=\pi_{j}^{ \pm 1}$.

Proof. None of the edges in $P_{i}$ are parallel in $\Gamma_{2}$ by Lemma 2.1, and similarly for $P_{j}$. Hence if $\pi_{i} \neq \pi_{j}^{ \pm 1}$, the edges in $P_{i}$ and $P_{j}$ define a subgraph $\Lambda$ of $\Gamma_{2}$ with $n$ vertices, $E=\alpha_{i}+\alpha_{j}$ pairwise non-parallel edges, and $F$ faces, say. Since $\chi\left(\widehat{F}_{2}\right)=0$ we have $F=E-n$, and since each face of $\Lambda$ has at least three sides we have $2 E \geq 3 F$. Hence $E \leq 3 n$.

The following lemma will also be used to eliminate some of the small cases in Lemma 7.15 .

Lemma 7.14. (i) $\pi_{i}=\pi_{i+1}$ if and only if $\alpha_{i}+\alpha_{i+1} \equiv 0(\bmod n)$

(ii) $\pi_{i}=\pi_{i+2}$ if and only if $\left(\alpha_{i}+\alpha_{i+1}\right)+\left(\alpha_{i+1}+\alpha_{i+2}\right) \equiv 0(\bmod n)$.

Proof. Recall that $\pi_{i}(x) \equiv x+p_{i}(\bmod n)$, for some $p_{i}, 1 \leq i \leq 4$. From Figure 60 we see that $p_{i+1} \equiv p_{i}-\left(\alpha_{i}+\alpha_{i+1}\right)(\bmod n)$. The result follows.

Lemma 7.15. If $\alpha_{i}, \alpha_{j} \geq n+1$ then $\pi_{i}=\pi_{j}^{ \pm 1}$. 
Proof. First we show that we may assume that some $\alpha_{i} \geq n+5$. For if not, then $\Delta n=\sum \alpha_{i} \leq 4(n+4)$, giving $(\Delta-4) n \leq 16$. Therefore either $\Delta=8$ and $n=4$, or $\Delta=7$ and $n=4$ or 5 , or $\Delta=6$ and $n=4,5,6,7$ or 8 . Now if $n=4$ or 6 , there is up to sign only one possible permutation with a single orbit, so the lemma is trivially true in this case.

First consider the case $\Delta=7, n=5$. Assuming that each $\alpha_{i} \leq n+4=9$, the only possibility for $\left(\alpha_{1}, \alpha_{2}, \alpha_{3}, \alpha_{4}\right)$ (up to cyclic permutation) is $(9,9,9,8)$. The result now follows from Lemma 7.13.

If $\Delta=6, n=8$, and each $\alpha_{i} \leq n+4=12$, then $\left(\alpha_{1}, \alpha_{2}, \alpha_{3}, \alpha_{4}\right)=(12,12,12,12)$. Hence $\pi_{1}=\pi_{2}=\pi_{3}=\pi_{4}$ by Lemma 7.14(i).

If $\Delta=6, n=7$, and each $\alpha_{i} \leq n+4=11$, then the only possibilities for $\left(\alpha_{1}, \alpha_{2}, \alpha_{3}, \alpha_{4}\right)$ (up to cyclic permutation) are $(11,11,11,9),(11,11,10,10)$ and $(11,10,11,10)$. In the first case, $\pi_{1}=\pi_{2}^{ \pm 1}=\pi_{3}^{ \pm 1}$ by Lemma 7.13 . Hence either $\pi_{1}=\pi_{2}$, or $\pi_{2}=\pi_{3}$, or $\pi_{1}=\pi_{3}$. But this contradicts Lemma 7.14. In the second case, $\pi_{1}=\pi_{2}^{ \pm 1}$ by Lemma 7.13, and by Lemma 7.14(i), $\pi_{2}=\pi_{3}$ and $\pi_{1}=\pi_{4}$ (and in fact $\pi_{1}=\pi_{2}^{-1}$ ). Finally, in the case $(11,10,11,10) \pi_{1}=\pi_{2}=\pi_{3}=\pi_{4}$ by Lemma 7.14(i).

Finally, if $\Delta=6, n=5$, then $\left(\alpha_{1}, \alpha_{2}, \alpha_{3}, \alpha_{4}\right)$ must be some permutation of $(9,9,9,3),(9,9,8,4),(9,9,7,5),(9,9,6,6),(9,8,8,5),(9,8,7,6),(9,7,7,7),(8,8,8,6)$ or $(8,8,7,7)$. In all cases except $(9,9,6,6),(9,8,7,6),(8,8,8,6)$ and $(8,8,7,7)$, the desired conclusion follows immediately from Lemma 7.13. In these remaining cases, Lemmas 7.13 and 7.14 give either the desired conclusion or a contradiction. For example, if $\left(\alpha_{1}, \alpha_{2}, \alpha_{3}, \alpha_{4}\right)=(9,7,8,6)$ then by Lemma 7.14 (i) we have $\pi_{1}=\pi_{4}$, $\pi_{2}=\pi_{3}$, and by Lemma 7.13, $\pi_{1}=\pi_{2}^{ \pm 1}$. On the other hand, for the case $(9,8,6,7)$, Lemmas 7.13 and 7.14(i) imply that $\pi_{1}=\pi_{2}^{-1}, \pi_{1}=\pi_{4}^{-1}$, hence $\pi_{2}=\pi_{4}$. But this is impossible by Lemma 7.14(ii). The other cases follow similarly.

We suppose, then, that we have $n+5$ parallel edges of $\Gamma_{1}$, say $A_{1}, A_{2}, \ldots, A_{n}$, $B_{1}, B_{2}, B_{3}, B_{4}, B_{5}$, in anticlockwise order around vertex + , with associated permutation $\pi$. Let $N$ be the annulus obtained by cutting $\widehat{F}_{2}$ along $A_{1}, A_{2}, \ldots, A_{n}$ in $\Gamma_{2}$. We will use $\ell$ to denote the vertex $\pi^{\ell}(1)$ of $\Gamma_{2}$. As mentioned above, we may assume that $n \geq 5$.

Suppose $\alpha_{i} \geq n+1$. We shall show that $\pi_{i}=\pi^{ \pm 1}$. So assume not. Then (since $\pi$ has only one orbit by Lemma 4.2) $\pi_{i}=\pi^{m}$ where $m \neq \pm 1$. Then $P_{i}$ contains edges $X$ and $Y$, each of which has (without loss of generality) label $\mathbf{0}$ at vertex + and label $\boldsymbol{m}$ at vertex -.

The essentially different possible arrangements of $X$ and $Y$ in $N$ are shown in Figure 61(a)-(f). The cases where $X$ and $Y$ are both of type I (as defined in the proof of Lemma 5.4) are shown in (a), (b), and (c); when both are of type II in (d) and (e), and when one is of type I and the other of type II, in (f).

First we dispose of case (a) by noting that it clearly precludes the existence of the edge in $P_{i}$ with label $\mathbf{1}$ at + and $\boldsymbol{m}+\mathbf{1}$ at - .

Now consider the edges $B_{1}, \ldots, B_{5}$. The edge $B_{j}$ joins vertices $\boldsymbol{k}_{\boldsymbol{j}}, \boldsymbol{k}_{\boldsymbol{j}}+\mathbf{1}$ of $\Gamma_{2}$, for some $k_{j}$, and of course $B_{j}$ is not parallel to $A_{j}$ in $\Gamma_{2}$ by Lemma 2.1, $1 \leq j \leq 5$.

Note that if $B_{1}$ (say) is of type I, then firstly, there is at most one other $B_{j}$ of type I (with its endpoints on the other component of $\partial N$ ), and secondly, any $B_{j}$ of type II must share an endpoint with $B_{1}$. It follows easily that $B_{j}$ is of type II, $1 \leq j \leq 5$. 


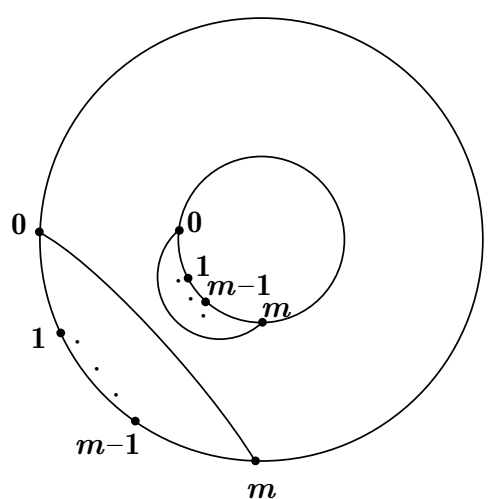

(a)

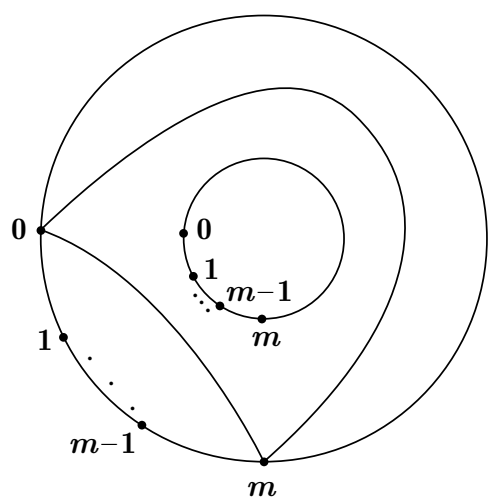

(c)

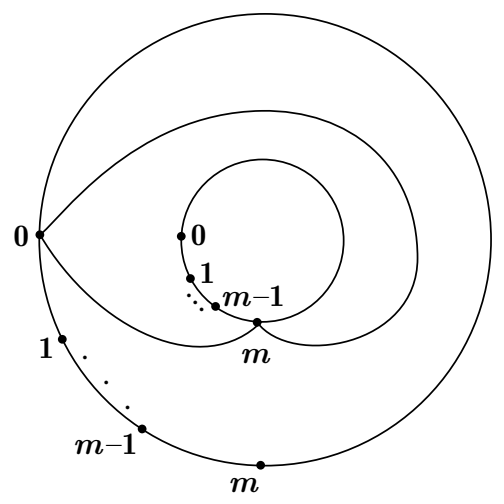

(e)

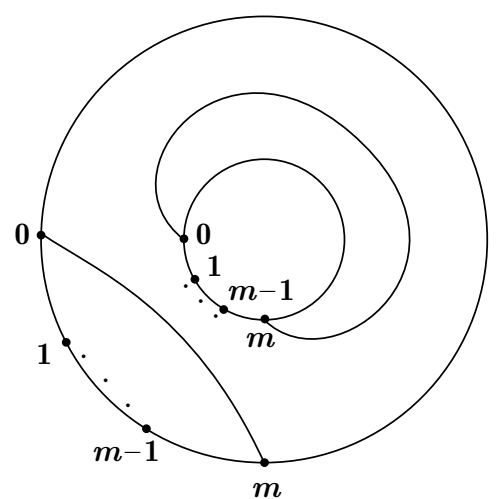

(b)

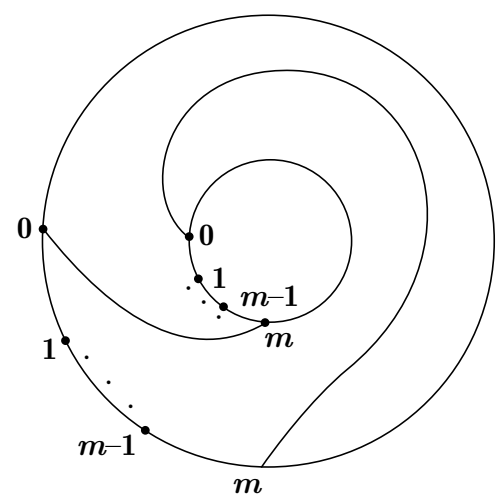

(d)

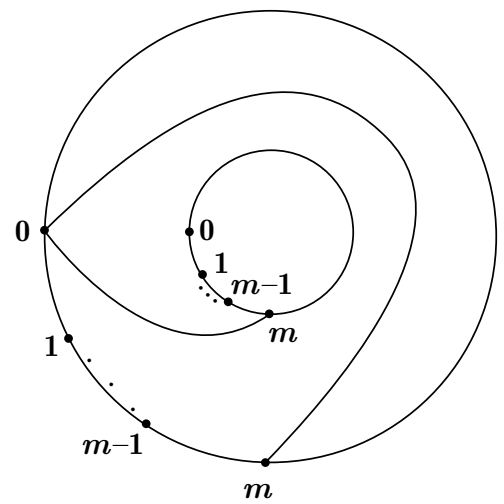

(f)

FiguRE 61 


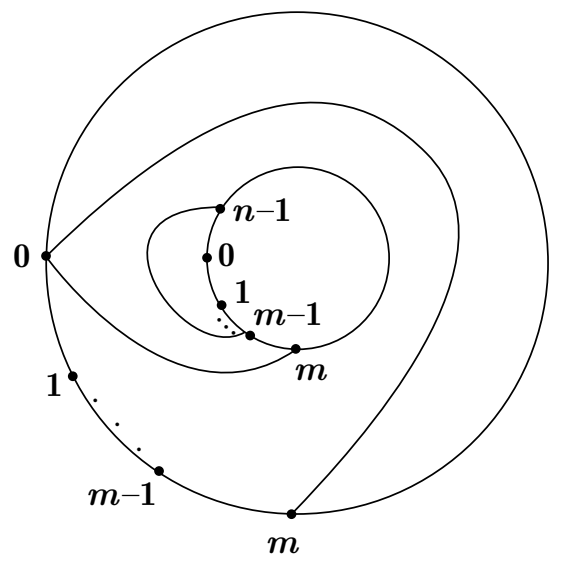

(i)

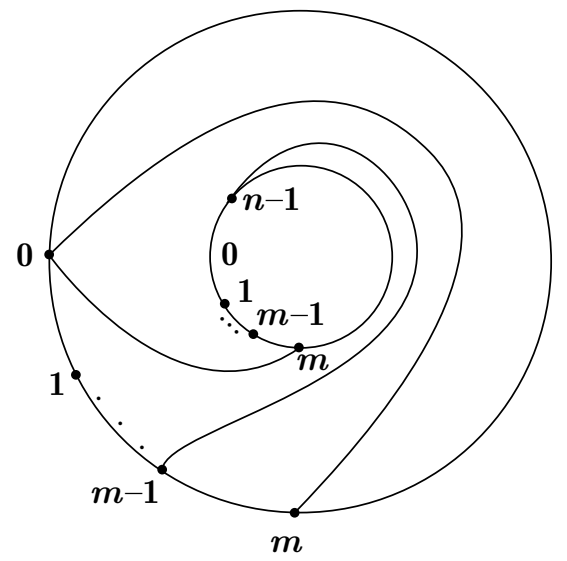

(ii)

FiguRe 62

Let the boundary components of $N$ be $\partial_{0} N$ and $\partial_{1} N$, illustrated in the figures as the outer and inner boundary components, respectively. Let $(\boldsymbol{k}, \boldsymbol{\ell})$ denote the pair of vertices $\boldsymbol{k}$ on $\partial_{0} N$ and $\boldsymbol{\ell}$ on $\partial_{1} N$. Thus the pair of endpoints of $B_{j}$ is either $\left(\boldsymbol{k}_{j}, \boldsymbol{k}_{\boldsymbol{j}}+\mathbf{1}\right)$ or $\left(\boldsymbol{k}_{\boldsymbol{j}}+\mathbf{1}, \boldsymbol{k}_{\boldsymbol{j}}\right)$. It is straightforward to check that in each of the cases (b), (c), (d) or (e) there can be at most four such edges disjoint from $X$ and $Y$. For instance, in case (b) the possible endpoint pairs are $(\boldsymbol{n}-\mathbf{1}, \mathbf{0}),(\mathbf{0}, \mathbf{1}),(\boldsymbol{m}, \boldsymbol{m}-\mathbf{1})$, and $(\boldsymbol{m}+\mathbf{1}, \boldsymbol{m})$. The other three cases are similar.

Finally, consider case (f). Let $Z$ be the edge in $P_{i}$ with label $\boldsymbol{n}-\mathbf{1}$ at vertex + and lable $\boldsymbol{m}-\mathbf{1}$ at vertex -. As it lies in $N, Z$ is either of type I or type II; the two possibilities are illustrated in Figures 62(i) and (ii) respectively. It is now easy to check that in each case there are at most four possible disjoint edges, disjoint from $X, Y$ and $Z$, with endpoints of the form $(\boldsymbol{k}, \boldsymbol{k}+\mathbf{1})$ or $(\boldsymbol{k}+\mathbf{1}, \boldsymbol{k})$.

In the sequel, $\pi$ will denote the permutation $\pi_{i}$ associated with some $P_{i}$ with $\alpha_{i} \geq n+1$.

Lemma 7.16. $\nu(x, \pi(x))=2$ for all vertices $x$ of $\Gamma_{2}$.

Proof. First note that we may suppose that $\alpha_{i} \leq n$ for at most one value of $i$. For suppose $\alpha_{1}, \alpha_{2} \leq n$ (say). Then $\alpha_{3}+\alpha_{4} \geq(\Delta-2) n$. Since $\alpha_{3}+\alpha_{4} \leq 4 n$ by Lemma 7.11, this is a contradiction unless $\alpha_{1}=\alpha_{2}=n, \alpha_{3}=\alpha_{4}=2 n$ (and $\Delta=6$ ). But this implies that $\pi_{1}=\pi_{2}=\pi_{3}=\pi_{4}$, contradicting Lemma 7.3.

So we may suppose that $\alpha_{1}, \alpha_{2}, \alpha_{3}, \geq n+1$. Hence $\pi_{i}=\pi^{ \pm 1}, i=1,2,3$, by Lemma 7.15.

If $\alpha_{4} \geq n+1$, then $\pi_{4}=\pi^{ \pm 1}$ also. Since not all the $\pi_{i}$ 's can be equal, by Lemma 7.3, we have (without loss of generality) that either $\pi_{1}=\pi_{2}=\pi_{3}^{-1}=\pi_{4}^{-1}$, or $\pi_{1}=\pi_{2}=\pi_{3}=\pi_{4}^{-1}$. In the first case, we may again suppose without loss of generality that $\alpha_{1}+\alpha_{2} \geq 3 n$, and thus every vertex $x$ of $\Gamma_{2}$ appears twice in either $P_{1}^{+}$or $P_{2}^{+}$. (Here $P_{i}^{+}$denotes the labels at the endpoints at vertex + of the edges in $P_{i}$.) Therefore $\nu(x, \pi(x)) \geq 2$ by Lemma 2.1. Hence $\nu(x, \pi(x))=2$ for all $x$, by Lemma 5.4(ii). In the second case, since $\alpha_{1}+\alpha_{2}+\alpha_{3} \geq 4 n$ by Lemma 7.11, every 
vertex $x$ of $\Gamma_{2}$ appears twice in either $P_{1}^{+}, P_{2}^{+}$or $P_{3}^{+}$. Therefore $\nu(x, \pi(x))=2$ for all $x$, as before.

Now suppose $\alpha_{4} \leq n$. Since $\pi_{i}=\pi^{ \pm 1}, i=1,2,3$, we may suppose without loss of generality that $\pi_{1}=\pi_{2}=\pi$. Since $\alpha_{3} \leq 2 n$ by Lemma 7.11, $\alpha_{1}+\alpha_{2} \geq 3 n$. Therefore $\nu(x, \pi(x))=2$ for all vertices $x$ of $\Gamma_{2}$, as above.

Lemma 7.17. If $\Delta=7$ then no three permutations $\pi_{i}$ are equal.

Proof. Suppose that $\pi_{1}=\pi_{2}=\pi_{3}=\pi$. Since $\alpha_{4} \leq 2 n$, we have $\alpha_{1}+\alpha_{2}+\alpha_{3} \geq 5 n$. Therefore, for all vertices $x$ of $\Gamma_{2}$, there are at least five edges in $\Gamma_{1}$ with label $x$ at + and label $\pi(x)$ at - . Since $\nu(x, \pi(x))=2$ by Lemma 7.16 , some three of these edges must belong to the same parallelism class in $\Gamma_{2}$. Since these edges all have label + at $x$ in $\Gamma_{2}$, this parallelism class would then contain at least five edges, contradicting (by Lemma 2.1) the fact that $\Gamma_{1}$ has only four parallelism classes of edges.

Lemma 7.18. $\Delta=7$ is impossible.

Proof. First note that we may assume that $\alpha_{i} \geq n+1, i=1,2,3,4$. For if $\alpha_{1}=n$ (say), then (by Lemma 7.11) we must have $\alpha_{2}=\alpha_{3}=\alpha_{4}=2 n$. This implies that $\pi_{1}=\pi_{2}=\pi_{3}=\pi_{4}$, contradicting Lemma 7.3.

By Lemma 7.15 we therefore have $\pi_{i}=\pi^{ \pm 1}, i=1,2,3,4$. By Lemma 7.17, two of these are $\pi$ and two are $\pi^{-1}$. Let $x$ be a vertex of $\Gamma_{2}$. Then, without loss of generality, at vertex + of $\Gamma_{1}$ the label $x$ appears exactly twice in each of the parallelism classes of edges with permutation $\pi^{-1}$, exactly twice in one of the parallelism classes with permutation $\pi$, and exactly once in the remaining parallelism class with permutation $\pi$.

Consider the corresponding edges in $\Gamma_{2}$, i.e., those with label + at vertex $x$. Note that in $\Gamma_{2}$, any edge incident to $x$ joins $x$ to either $\pi(x)$ or $\pi^{-1}(x)$, and, by Lemma 7.16, $\nu(x, \pi(x))=\nu\left(x, \pi^{-1}(x)\right)=2$. Hence, by Lemma 2.1, of the edges with label + at $x$, exactly two belong to each of the parallelism classes joining $x$ to $\pi^{-1}(x)$, exactly two belong to one of the parallelism classes joining $x$ to $\pi(x)$, and exactly one to the other such parallelism class. Let these last three edges be $A, B$ and $C$ respectively. Then around vertex $x$ these edges appear as in Figure 63(i), (ii) or (iii).

A similar argument applied to the edges of $\Gamma_{2}$ with label - at vertex $\pi(x)$ shows that the edges $A, B$ and $C$ appear at vertex $\pi(x)$ as in Figure 64(i), (ii) or (iii). (These possibilities are independent of the possible arrangements at vertex $x$.)

Now in $\Gamma_{1}, C$ is parallel to either $A$ or $B$. On the other hand, from Figures 63 and 64 we see that if $A(+)$ denotes the endpoint of $A$ with label + , etc., then (with suitable choice of orientation) we have $\delta_{2}(A(+), C(+))=4,8$ or $12, \delta_{2}(A(-), C(-))=$ 10,6 or 2 , and $\delta_{2}(B(+), C(+))=2,6$ or $10, \delta_{2}(B(-), C(-))=12,8$ or 4 . This contradicts Lemma 2.5(i).

Lemma 7.19. $\Delta=6$ is impossible.

Proof. First note that at most one $\alpha_{i}$ is $\leq n$. For if $\alpha_{1}, \alpha_{2} \leq n$ (say), then $\alpha_{3}+\alpha_{4} \geq$ $4 n$, and therefore, by Lemma 7.11, $\alpha_{1}=\alpha_{2}=n$, and $\alpha_{3}=\alpha_{4}=2 n$. But this implies that $\pi_{1}=\pi_{2}=\pi_{3}=\pi_{4}$, contradicting Lemma 7.3.

So suppose $\alpha_{1}, \alpha_{2}, \alpha_{3} \geq n+1$. Then $\pi_{i}=\pi^{ \pm 1}, i=1,2,3$. Note that $\alpha_{4}>0$, for if not then we would have $\alpha_{1}=\alpha_{2}=\alpha_{3}=2 n$, contradicting (the proof of) Lemma 7.3. Also (by re-choosing $\alpha_{4}$ if necessary) we may assume that $\alpha_{4}<2 n$. 


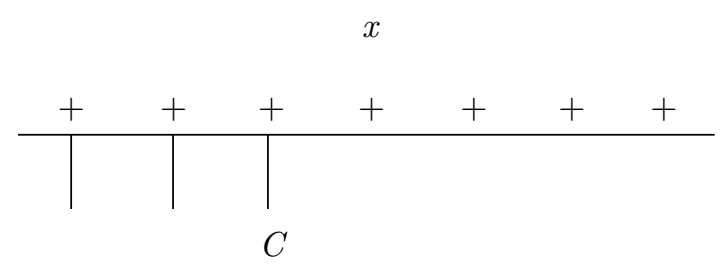

(i)

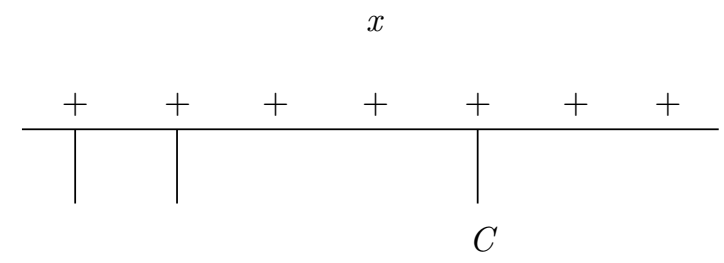

(ii)

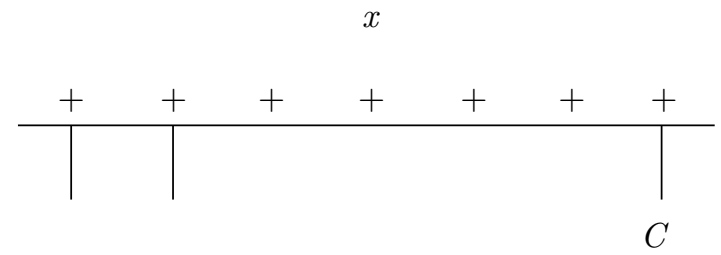

(iii)

FigURE 63 


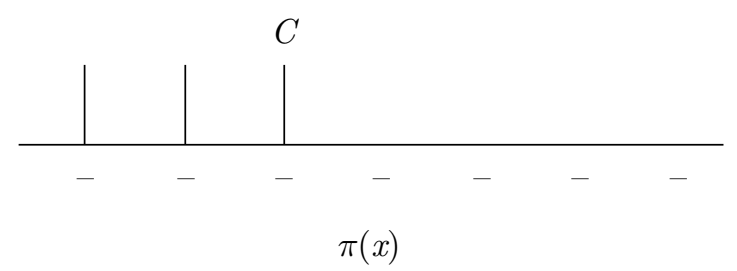

(i)

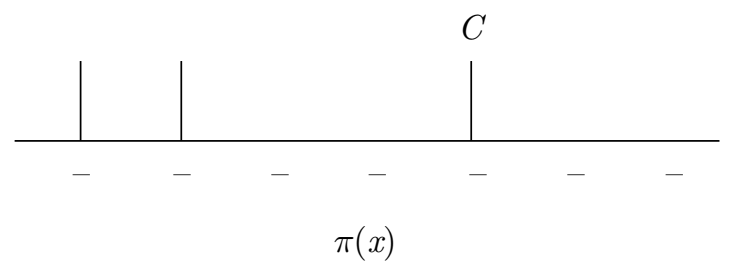

(ii)

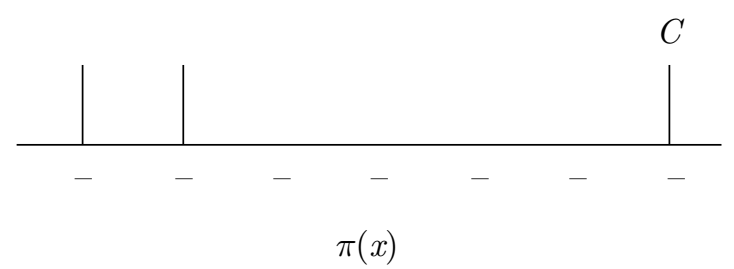

(iii)

FiguRE 64 


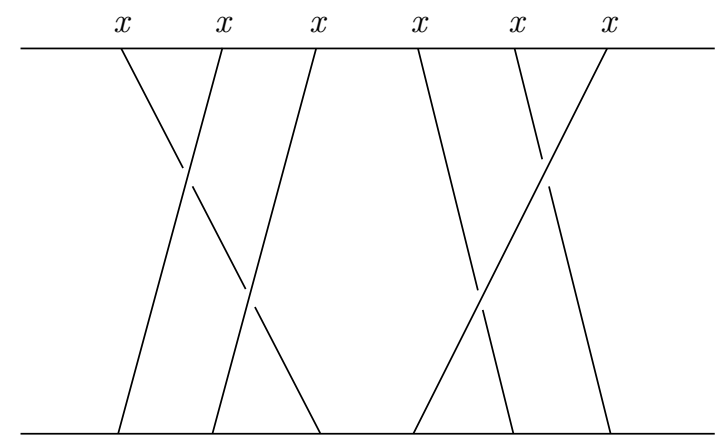

FIGURE 65

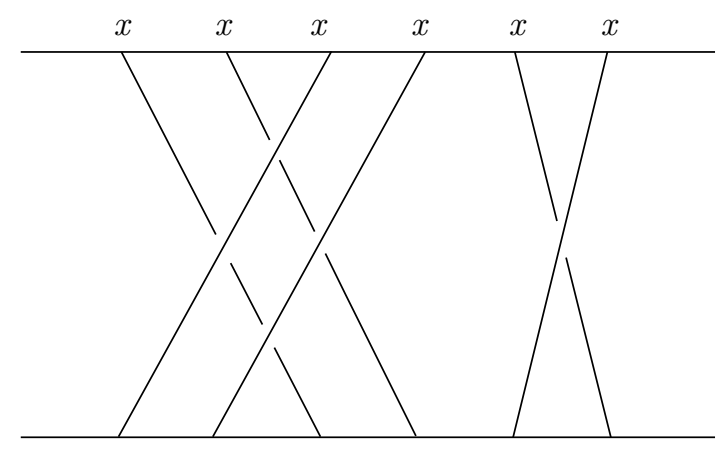

FigURE 66

Let $x$ be a label that appears exactly once in $P_{4}^{+}$. Then $x$ appears exactly once in one of $P_{1}^{+}, P_{2}^{+}, P_{3}^{+}$, and exactly twice in the two others. The three possible arrangements are shown in Figures 65, 66 and 67. In particular, there are at least three edges in $P_{1} \cup P_{2} \cup P_{3}$ with label $x$ at + and (say) $\pi(x)$ at -. By Lemma 7.16, two of these, say $A$ and $B$, are parallel in $\Gamma_{2}$. Since the number of edges in any parallelism class in $\Gamma_{2}$ is at most four, $A$ and $B$ are adjacent around $x$ among the edges of $\Gamma_{2}$ with label + at $x$. Since $d=1, A$ and $B$ are adjacent at + among those edges in $\Gamma_{1}$ with label $x$ at + . But examination of Figures 65,66 and 67 shows that any such adjacent pair of edges violates either Lemma 2.1 or Lemma 2.5(i).

We have thus shown that in Case (B), the only possibilities for $F_{1}, F_{2}$ are given by the identification patterns $P(7)$ and $P(6)_{2}$.

\section{CASe (C)}

In this section we treat the case where $\Delta=6, n_{1} \geq 3, n_{2}=2$, the two boundary components of $F_{2}$ are of opposite sign, and $F_{2}$ is bad.

It follows from Lemma 3.2 that the arcs of $F_{1} \cap F_{2}$ in $F_{2}$ come in parallelism classes of size $n_{1}$. Thus $\Gamma_{2} \cong G\left(n_{1}, n_{1}, n_{1}, n_{1}, n_{1}\right)$. Call the boundary components of $F_{2}$ (vertices of $\left.\Gamma_{2}\right)+$ and - . The $n_{1}$ loops at vertex + in $\Gamma_{2}$ define a permutation $\pi$ of $\left\{1,2, \ldots, n_{1}\right\}$ with orbits of size 2 (thus $n_{1}$ is even). Since $n_{1} \geq 3$, there are at least two such orbits. This gives edges in $\Gamma_{1}$ as shown in Figure 68. Write $n_{1}=2 n$. 


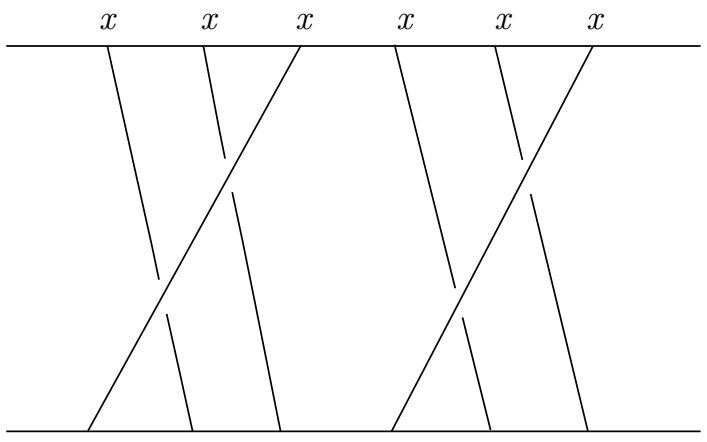

FigURE 67

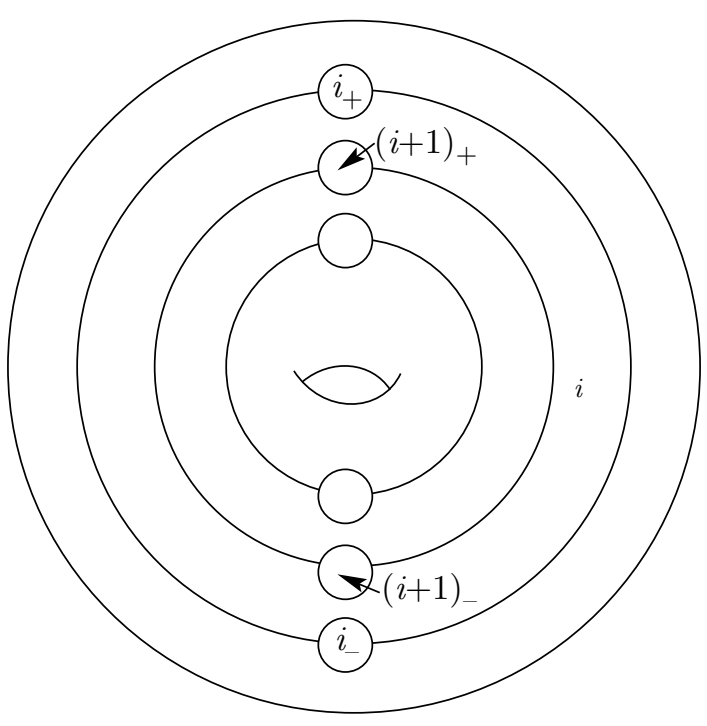

FiguRe 68

The four parallelism classes of edges in $\Gamma_{2}$ that run from + to - all define the same permutation $\rho$; that is, if an edge has label $i$ at vertex + , then it has label $\rho(i)$ at vertex - . Note that the elements in any orbit of $\rho$ all have the same sign, and that the corresponding cycle must be essential in $\widehat{F}_{1}$ by Lemma 2.3 .

Let the orbits of $\pi$ be $\left\{i_{+}, i_{-}\right\}, i=1,2, \ldots, n$. The corresponding edges of $\bar{\Gamma}_{1}$ decompose $\widehat{F}_{1}$ into annuli $N_{1}, N_{2}, \ldots, N_{n}$, numbered so that $\partial N_{i}$ contains the four vertices $i_{ \pm},(i+1)_{ \pm}$of $\Gamma_{1}$ (see Figure 68). The only vertices of the same sign accessible from $i_{+}$are $(i \pm 1)_{+}$and $i_{+}$. Hence either

(1) $\rho$ is the identity, or

(2) $\rho\left(i_{+}\right)=(i+1)_{+}$, say, and $\rho\left(i_{-}\right)=(i+\varepsilon)_{-}$, where $\varepsilon= \pm 1$, so $\rho$ has exactly two orbits $\left\{i_{+}: i=1,2, \ldots, n\right\}$ and $\left\{i_{-}: i=1,2, \ldots, n\right\}$.

In case (1), consider one of the corresponding loops in $\Gamma_{1}$, at vertex $i_{+}$, say. This must lie in either $N_{i}$ or $N_{i-1}$, and there cannot be such loops in both $N_{i}$ and $N_{i-1}$, as this would prohibit the existence of a loop at $i_{-}$. Hence if we let $A_{1}, A_{2}, \ldots, A_{6}$ 


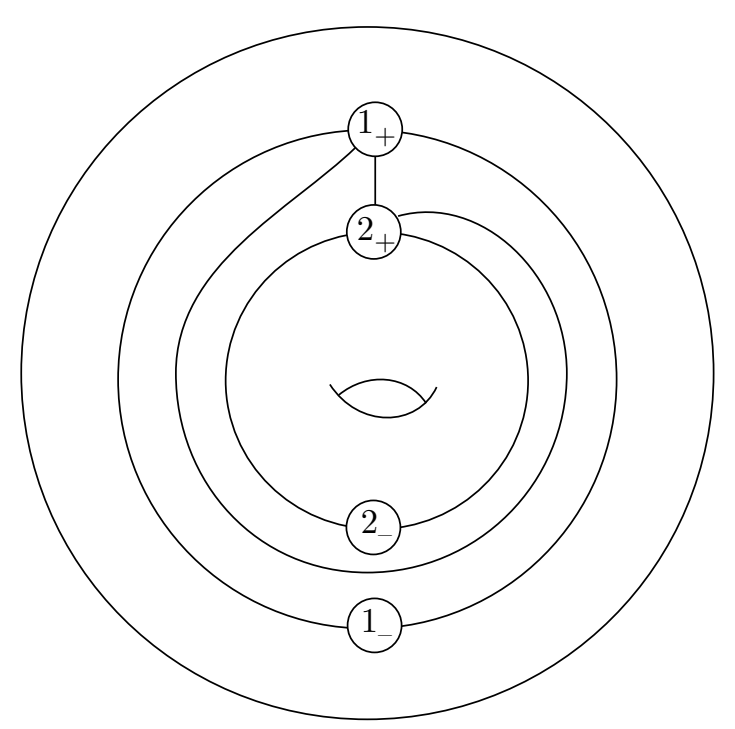

FIGURE 69

be the edges of $\Gamma_{1}$ with label + , say, at the vertex $i_{+}$, then they may be numbered consecutively around $i_{+}$in such a way that $A_{1}$ and $A_{6}$ join $i_{+}$to $i_{-}=\pi\left(i_{+}\right)$, and $A_{2}, A_{3}, A_{4}$, and $A_{5}$ join $i_{+}$to $i_{+}=\rho\left(i_{+}\right)$. Since $d=d_{12}=1$, these edges appear in the same order around vertex + in $\Gamma_{2}$. Thus, there, $A_{1}$ and $A_{6}$ are loops at + while $A_{2}, A_{3}, A_{4}$, and $A_{5}$ join vertices + and -. But this contradicts the form of $\Gamma_{2}$.

In case (2), we distinguish the two subcases (a) $n_{1} \geq 6$, and (b) $n_{1}=4$.

First consider (a). Here, any edge of $\bar{\Gamma}_{1}$ joining vertices $i_{+}$and $\rho\left(i_{+}\right)=(i+1)_{+}$ must lie in the annulus $N_{i}$. Hence there is at most one such edge, as otherwise there could be no edge joining $i_{-}$and $(i+1)_{-}$. The four edges of $\Gamma_{2}$ with label $i_{+}$ at + and $\rho\left(i_{+}\right)$at - are therefore all parallel in $\Gamma_{1}$. But this implies the existence of an edge in $\Gamma_{1}$ with label - at $i_{+}$and label + at $\rho\left(i_{+}\right)$. Considering this edge in $\Gamma_{2}$ shows that $\rho^{2}$ is the identity, contradicting our assumption that $n_{1} \geq 6$.

Finally, consider subcase (b). Let $X_{1}, X_{2}$ be two edges of $\bar{\Gamma}_{1}$ joining vertices $1_{+}$ and $2_{+}$that come from some parallelism class in $\Gamma_{2}$ with corresponding permutation $\rho$. If these both lie in $N_{1}$, say (see Figure 69), then the corresponding edges joining $1_{-}$and $2_{-}$must lie in $N_{2}$, and hence $X_{1}, X_{2}$ are the only edges of $\bar{\Gamma}_{1}$ joining $1_{+}$ and $2_{+}$. Thus the arrangement of the edges $A_{1}, A_{2}, \ldots, A_{6}$ in $\Gamma_{1}$ with label + at vertex $1_{+}$around that vertex is as described in case (1) above, giving the same contradiction.

If $X_{1}, X_{2}$ lie in $N_{1}, N_{2}$ respectively, then $\bar{\Gamma}_{1}$ is as shown in Figure 70 . Choose the numbering of the labels around vertex + of $\Gamma_{2}$ so that each parallelism class of edges has labels 1,2,3,4 there, in anticlockwise order. Then we must have $\rho(i) \equiv i+2$ $(\bmod 4)$, and so the labels in any parallelism class at vertex - are (in clockwise order) $3,4,1,2$. Now let $A$ be one of the loops at vertex + in $\Gamma_{2}$ with endpoint labels 1 and 4, and let $X, Y$ be the two loops at vertex - with endpoint labels 1 and 4. See Figure 71. Then, since there are exactly two parallelism classes of edges in $\Gamma_{1}$ joining vertices 1 and $4, A$ must be parallel in $\Gamma_{1}$ to either $X$ or 


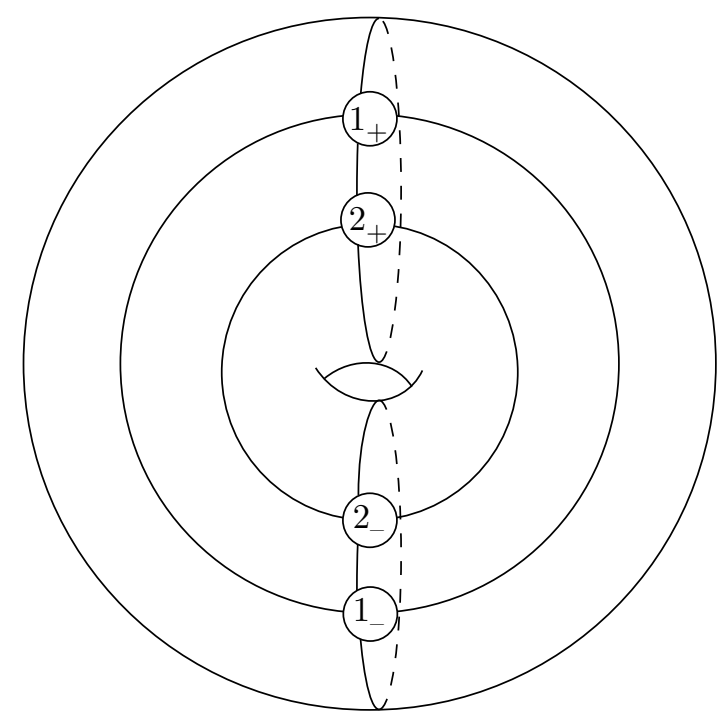

FiguRe 70

$Y$. But if $A(i)$ denotes the endpoint of $A$ in $\Gamma_{2}$ with label $i$, and similarly for $X$ and $Y$, then we have (for suitable choice of orientation) $\tau_{2}(A(1), A(4))=15$, $\tau_{2}(X(1), X(4))=\tau_{2}(Y(1), Y(4))=11$. This contradicts Lemma 2.5(iii).

We have thus shown that Case $(\mathrm{C})$ cannot occur.

\section{CAse (D)}

In this section we treat the case where $\Delta=6, n_{1} \geq 3$, all the boundary components of $F_{2}$ have the same sign, and $F_{2}$ is bad.

It follows from Lemma 3.2 that the edges of $\Gamma_{2}$ come in parallelism classes of size $n_{1}$. Each such parallelism class defines a permutation $\rho$ with orbits of size 2 . Thus $n_{1}$ is even and the number of orbits of $\rho$ is $n_{1} / 2 \geq 2$. The parallelism class gives rise to edges in the reduced graph $\bar{\Gamma}_{1}$ as shown in Figure 68. It is then easy to see that $\bar{\Gamma}_{1}$ is a subgraph of either the graph illustrated in Figure 72, or, when $n_{1}=4$, the graph illustrated in Figure 73. In particular, there are at most two permutations $\rho$.

We distinguish the three cases $n_{2}=1, n_{2}=2$, and $n_{2} \geq 3$.

CASE $(1) . n_{2}=1$. Here $\Gamma_{2} \cong H\left(n_{1}, n_{1}, n_{1}\right)$, and there is only one permutation $\rho$. Thus $\bar{\Gamma}_{1}$ is as shown in Figure 68. Since $d=1$, this determines the identification between the edges of $\Gamma_{1}$ and $\Gamma_{2}$ (modulo permutation of the orbits of $\rho$ on $\widehat{F}_{1}$ ), namely that given by the identification pattern $P(6)_{2 n}$ (where $\left.n_{1}=2 n\right), n \geq 2$, shown in Figure 74. Although this case is combinatorially possible, we shall see in Section 11 that it is actually topologically degenerate.

CASE $(2) . n_{2}=2$. Here $\Gamma_{2} \cong G\left(n_{1}, n_{1}, n_{1}, n_{1}, n_{1}\right)$. Let the vertices of $\Gamma_{2}$ be $x$ and $y$. Without loss of generality, suppose that the labels at the ends of any parallelism class of edges at the vertex $x$ are, in order, $1,2, \ldots, n_{1}$. Let the labels at the vertex $y$ be $m, m+1, \ldots, m-1$. Then the permutations defined by the loops 
C. McA. GORDON
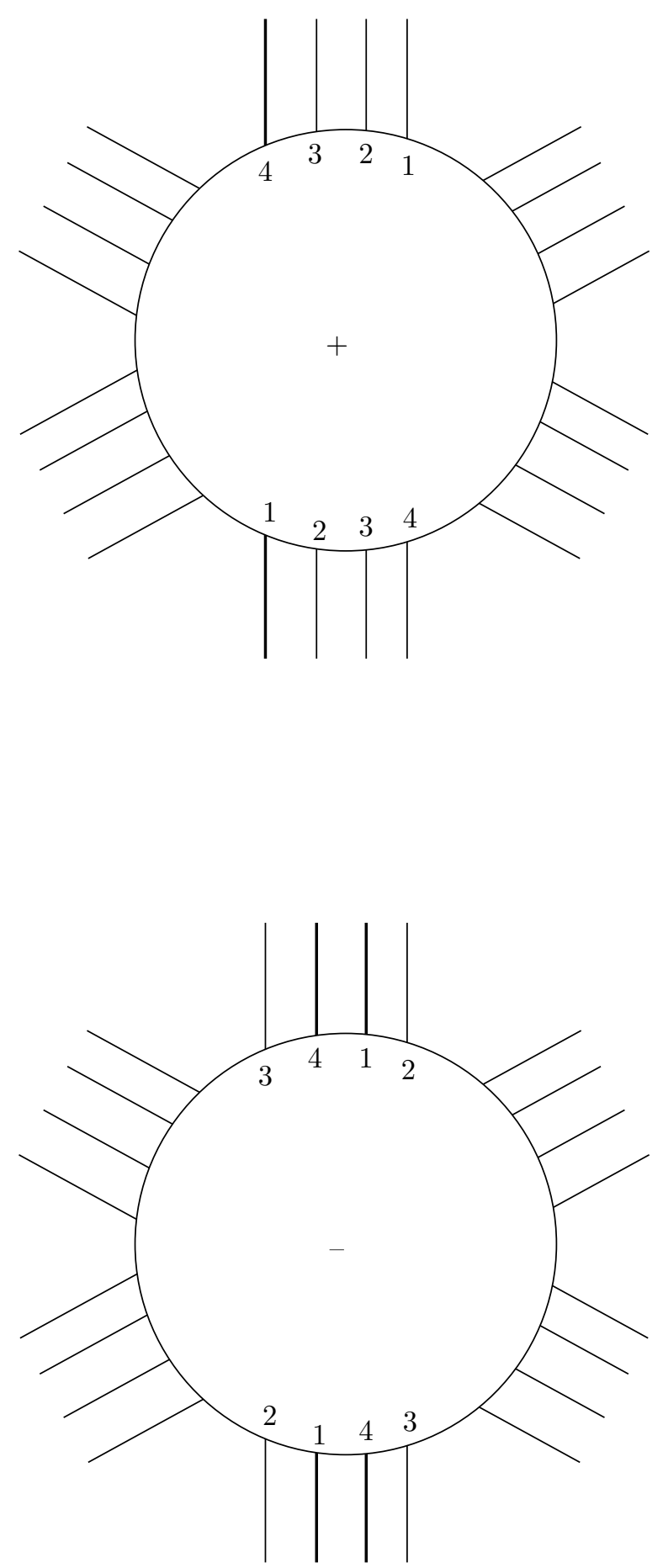

FIGURE 71 


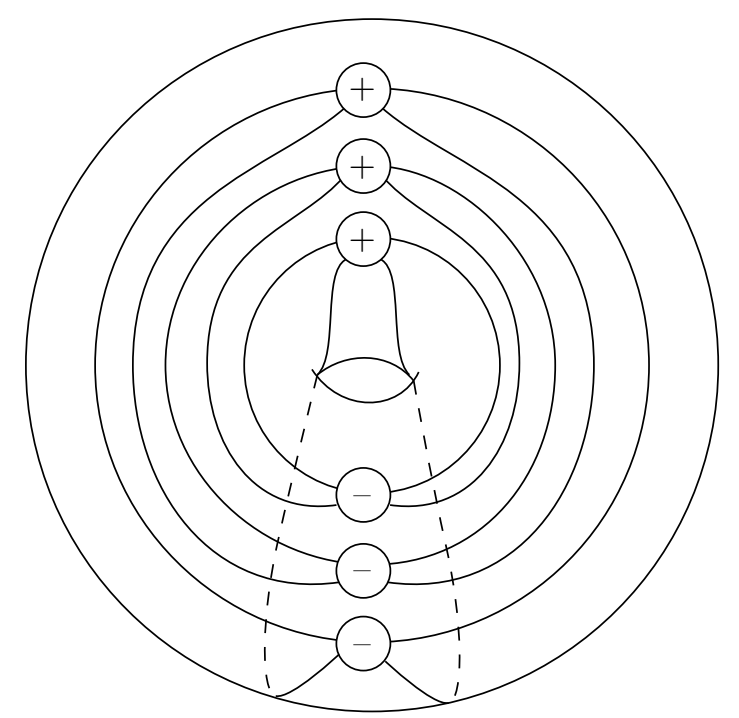

FiguRE 72

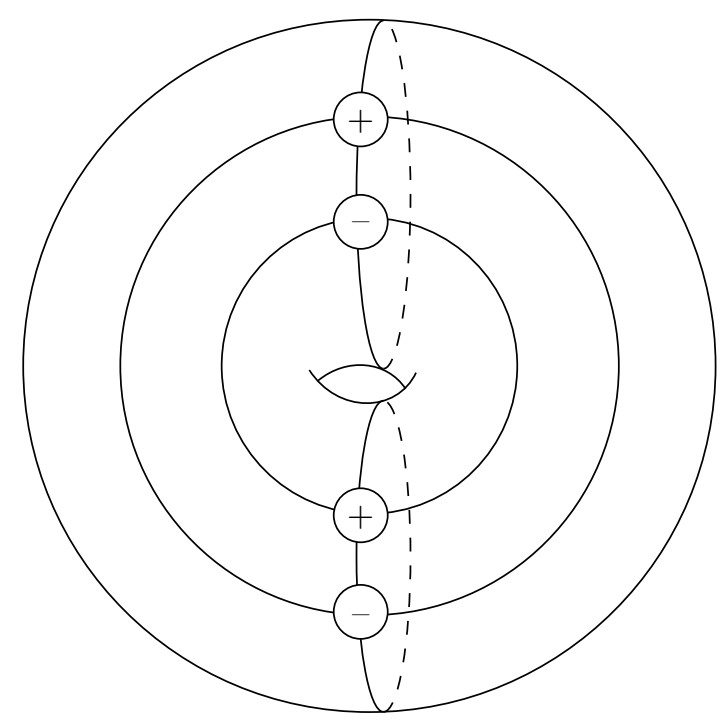

FigURE 73

at $x$, the loops at $y$, and the edges joining $x$ and $y$, respectively, are

$$
\begin{aligned}
& \rho_{1}: i \mapsto 1-i, \\
& \rho_{2}: i \mapsto 2 m-1-i, \\
& \rho_{3}: i \mapsto m-i .
\end{aligned}
$$

By the remarks at the beginning of the section, at least two of these permutations must coincide. 
C. McA. GORDON

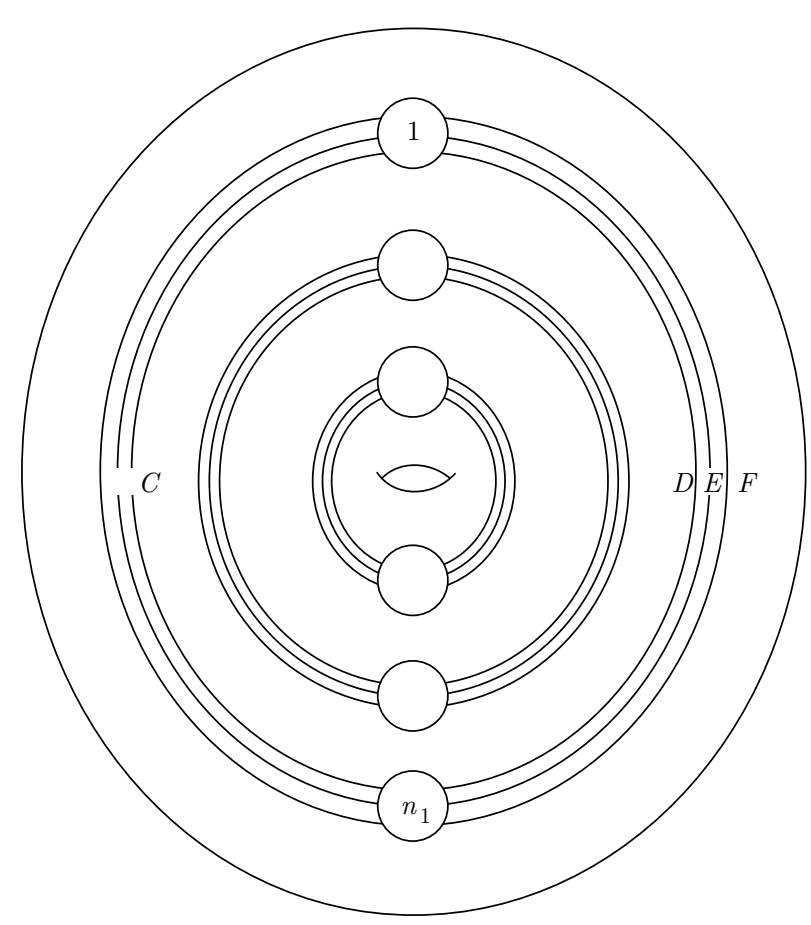

(i)

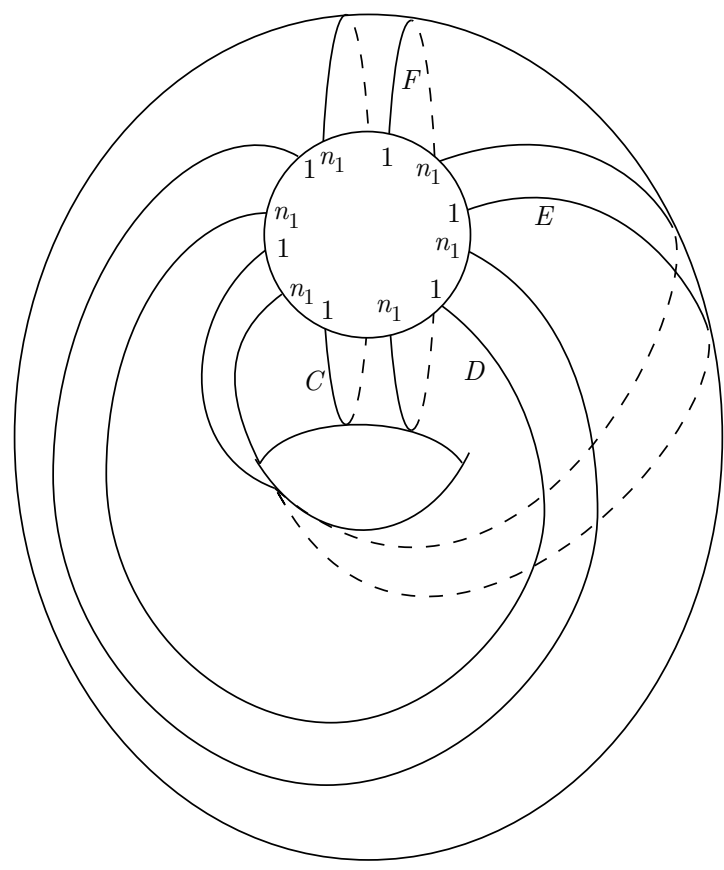

(ii)

FiguRe 74 

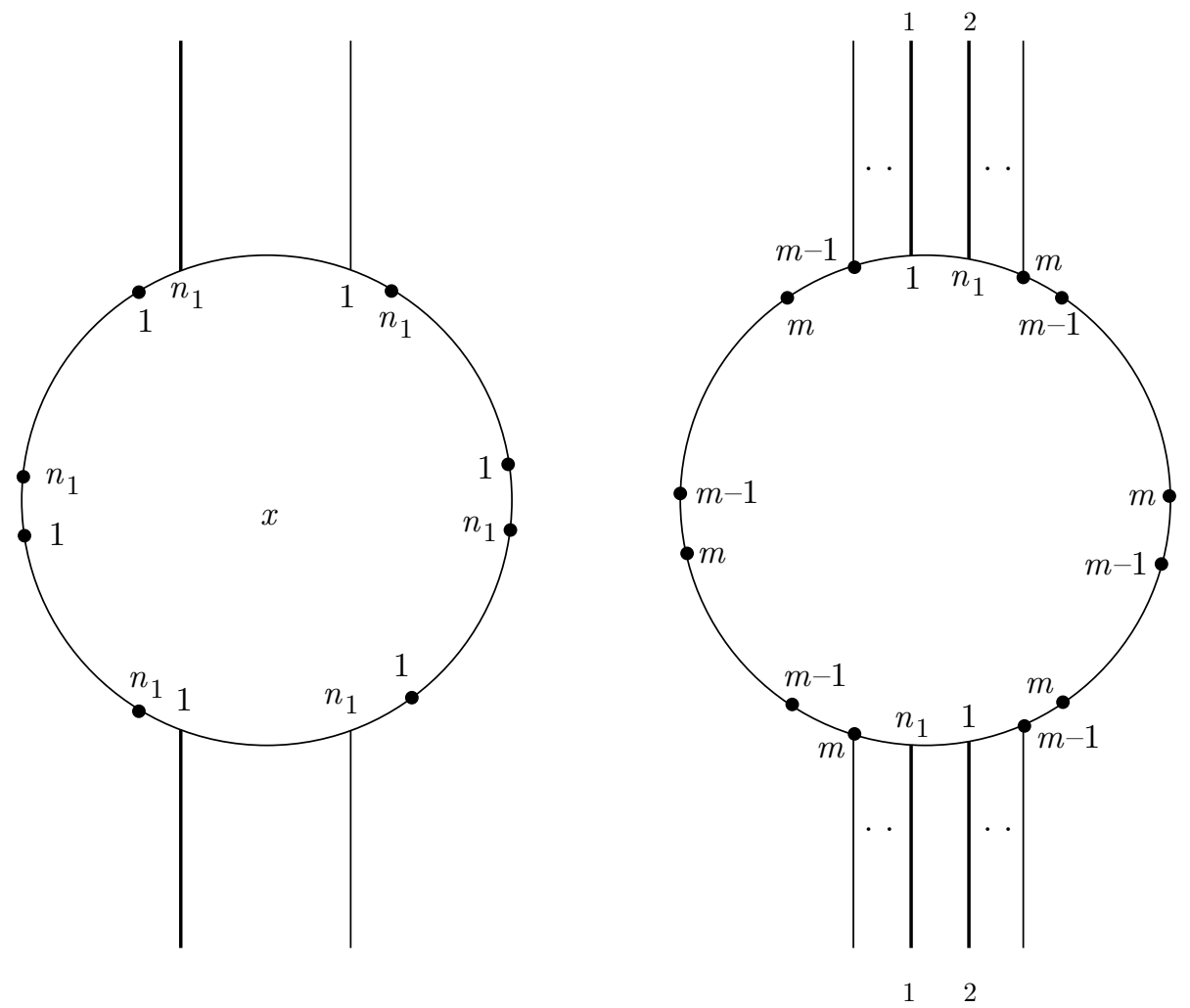

FIGURE 75

First suppose that all three permutations are equal, that is, $m=1$. A loop in $\Gamma_{2}$ gives rise to an edge in $\Gamma_{1}$ joining vertices $i$ and $1-i$, say, with equal labels at its two endpoints. But this implies (see Figure 68) that every edge in $\Gamma_{1}$ has equal labels at its endpoints, i.e., that $\Gamma_{2}$ consists entirely of loops. This is a contradiction.

If exactly two permutations coincide, then it is easy to see that they must be $\rho_{1}$ and $\rho_{2}$, and $m=\left(n_{1} / 2\right)+1$. Let $X$ be one of the loops in $\Gamma_{2}$ based at $x$ with endpoint labels 1 and $n_{1}$, and let $Y_{1}, Y_{2}$ be the loops based at $y$ with endpoint labels 1 and $n_{1}$ (see Figure 75). Since there are only two parallelism classes of edges in $\Gamma_{1}$ joining vertices 1 and $n_{1}, X$ must be parallel in $\Gamma_{1}$ to either $Y_{1}$ or $Y_{2}$. But this is impossible by Lemma 2.5(iii), since, if $X(i)$ denotes the endpoint of $X$ in $\Gamma_{2}$ with label $i$, and similarly for $Y_{1}$ and $Y_{2}$, then $\tau_{2}\left(X(1), X\left(n_{1}\right)\right)=4 n_{1}-1$ (say), while $\tau_{2}\left(Y_{j}(1), Y_{j}\left(n_{1}\right)\right)=3 n_{1}-1, j=1,2$.

CASE (3). $n_{2} \geq 3$. First suppose that there is only one permutation $\rho$. Then the edges in $\Gamma_{1}$ joining any vertex $a$ to $\rho(a)$ fall into two parallelism classes, each of size $3 n_{2}$. If $n_{2} \geq 4$, this contradicts Corollary 5.5. In any case, each of the parallelism classes defines a permutation $\pi$ of $\left\{1,2, \ldots, n_{2}\right\}$ which by Lemma 4.2 has only one orbit. Let $A_{1}, A_{2}, A_{3}, B_{1}, B_{2}, B_{3}$ be the edges with label $x$ at some vertex $a$, and label $\pi(x)$ at $\rho(a)$, for some $x$, numbered cyclically around $a$ in such a way that the $A_{i}$ 's belong to one parallelism class and the $B_{i}$ 's to the other. Since $d=1$, these edges appear in the same order around the vertex $x$ in $\Gamma_{2}$. Since $\nu(x, \pi(x)) \leq 5$ by 
Lemma 5.4(i), some consecutive pair must be parallel in $\Gamma_{2}$. But this is impossible by Lemmas 2.1 and $2.5(\mathrm{i})$.

Finally, suppose that there are two permutations $\rho$, say $\rho_{1}$ and $\rho_{2}$. First note that since the valency of each vertex $a$ in $\bar{\Gamma}_{1}$ is 4 , some parallelism class of edges in $\Gamma_{1}$ joining $a$ to $\rho_{1}(a)$, say, has at least $n_{2}+1$ members. By Lemma 4.2 , the corresponding permutation $\pi$ has only one orbit. Let the first $n_{2}$ of these edges be $A_{1}, A_{2}, \ldots, A_{n_{2}}$, where (without loss of generality) $A_{i}$ has label $i$ at $a$ and label $\pi(i)$ at $\rho_{1}(a)$.

For any vertex of $\Gamma_{2}$, the labels at the end of each parallelism class of edges incident at that vertex are, in order, $m, m+1, \ldots, m-1$ for some $m$. We may suppose that at vertex 1 , say, $m=1$. If $m=1$ for all vertices of $\Gamma_{2}$, then we get only one permutation $\rho$, contrary to hypothesis. Since the edges $A_{1}, A_{2}, \ldots, A_{n_{2}}$, as they lie in $\Gamma_{2}$, have label $a$ at vertex $i$ and label $\rho_{1}(a)$ at $\pi(i)$ for all $i$, we see that, at the vertex $\pi^{k}(1), m=1$ if $k$ is even and $m=m_{0}$ if $k$ is odd, where $m_{0} \neq 1$ is independent of $k$. In particular it follows that $n_{2}$ is even, and hence $\geq 4$.

Since this argument applies to any parallelism class of edges in $\Gamma_{1}$ with at least $n_{2}+1$ members, it also follows that there can be no such parallelism class joining vertices $a$ and $\rho_{2}(a)$. Hence if the two parallelism classes joining $a$ to $\rho_{i}(a)$ each have $\alpha_{i}$ members, $i=1,2$, then $\alpha_{2} \leq n_{2}$. Also, $2 \alpha_{1}+2 \alpha_{2}=6 n_{2}$. Hence, $\alpha_{2}<n_{2}$ implies $\alpha_{1}>2 n_{2}$, contradicting Corollary 5.5. We must therefore have $\alpha_{1}=2 n_{2}$, $\alpha_{2}=n_{2}$. It follows that any edge in $\Gamma_{1}$ joining $a$ and $\rho_{1}(a)$ has label $i$ at $a$ and label $\pi(i)$ at $\rho_{1}(a)$, say. But from the form of $\Gamma_{2}$ the edges in $\Gamma_{1}$ joining $a$ and $\rho_{1}(a)$ come in pairs, one with label $i$ at $a$ and label $\pi(i)$ at $\rho_{1}(a)$ and the other with label $\pi(i)$ at $a$ and label $i$ at $\rho_{1}(a)$. This implies that $\pi^{2}$ is the identity, and hence $n_{2}=2$, contrary to hypothesis.

We have thus shown that in Case (D), the only possibilities for $F_{1}, F_{2}$ are given by the identification patterns of the form $P(6)_{2 n}, n \geq 2$.

\section{Case (E)}

In this section we treat the case where $\Delta=6$ and $F_{1}$ and $F_{2}$ are bad.

By Lemma 3.2, the edges of $\Gamma_{\alpha}$ come in parallel families of size $n_{\beta}$. Also, since (again by Lemma 3.2) $\Gamma_{\alpha}$ has a face that is a disk with three sides, there is a family of $n_{\beta}$ parallel edges in $\Gamma_{\alpha}$ joining boundary components of the same sign. The orbits of the corresponding permutation each contain two vertices of $\Gamma_{\beta}$, of opposite sign. There are thus three cases:

(1) $n_{1}=n_{2}=2$;

(2) $n_{1}=2, n_{2} \geq 4$

(3) $n_{1}, n_{2} \geq 4$.

(1) is treated under Case (A), and (2) under Case (C), so it remains to consider (3).

By Lemma 2.3, a family of $n_{\beta}$ parallel edges in $\Gamma_{\alpha}$ joining vertices of the same sign gives a set of edges in $\Gamma_{\beta}$ as shown in Figure 68. Now consider a family of $n_{\beta}$ parallel edges in $\Gamma_{\alpha}$ joining vertices of opposite sign. As in Section 8 (Case $(\mathrm{C})$ ), the corresponding permutation either is the identity or has exactly two orbits. Again arguing as in Section 8 , we see that the number of edges of $\bar{\Gamma}_{\beta}$ joining vertices of the same sign is $n_{\beta}$. Since the edges of $\Gamma_{\beta}$ come in parallel families of size $n_{\alpha}$, this shows that the number of edges in $\Gamma_{\beta}$ joining vertices of the same sign is $n_{1} n_{2}$. 
But the total number of edges is $\frac{6 n_{1} n_{2}}{2}=3 n_{1} n_{2}$. Therefore, by the parity rule, either $\Gamma_{1}$ or $\Gamma_{2}$ has at least $\frac{3 n_{1} n_{2}}{2}$ edges joining vertices of the same sign. This contradiction shows that case (3) cannot occur.

Hence Case (E) is impossible.

\section{Final topological arguments}

We have shown that if $\Delta \geq 6$ and $(M, T)$ is not cabled, then, up to homeomorphism, the only possibilities for $\left(F_{1}, F_{2} ; F_{1} \cap F_{2}\right)$ are those described by the identification patterns $P(6), P(6)_{2 n}, P(7), P(8)_{1}$, and $P(8)_{2}$, illustrated in Figures $28,74,35,31$, and 32 . (Note that since in all cases the complementary regions on at least one of the surfaces are all disks, we may assume by a standard innermost circle argument that there are no circles of intersection.) In each of these cases, let $X=F_{1} \cup F_{2} \cup T$, and let $N(X)$ be an abstract regular neighborhood of $X \operatorname{rel} T$.

First we show that the family $P(6)_{2 n}$ is topologically degenerate.

Lemma 11.1. For $P(6)_{2 n}, n \geq 1, F_{1}$ is compressible in $N(X)$.

Proof. Let $D_{0}$ be a disk on $T$ of the form shown in Figure 76, containing $\partial F_{1} \cap \partial F_{2}$. We use $D_{0}$ as "base-point" for computations in $\pi_{1}(X)$. We choose $D_{0}$ so that the arc $u$ on boundary component 1 of $F_{1}$ which does not lie in $D_{0}$ appears on $F_{1}$ as shown in Figure 77 . Then the arc $v$ on boundary component $2 n$ shown in Figure 76 appears on $F_{1}$ as in Figure 77 (see Figure 74(ii)).

Let $a, b, c, d, e, f$ be the elements of $\pi_{1}(X)$ represented by the $\operatorname{arcs} A, B, C, D, E$, $F$, with the orientations indicated. Then, from the two 3 -sided faces on $F_{2}$ (see Figure 74(ii)) we get the relations

$$
\text { ace }=1, \quad b d f=1,
$$

and from the two bigons on $F_{1}$ bounded by the arcs $A, B$ and $E, F$ respectively, we get the relations

$$
a=b, \quad e=f .
$$

Therefore $c=d$. But the curve $\gamma$ shown in Figure 77 is essential on $F_{1}$ and represents $c d^{-1}$ in $\pi_{1}(X)$. Hence the map $\pi_{1}\left(F_{1}\right) \rightarrow \pi_{1}(X)$ is not injective.

Let $\partial_{0} N(X)$ denote $\partial N(X)-T$.

Lemma 11.2. For $P(6), P(7), P(8)_{1}$, and $P(8)_{2}$, every component of $\partial_{0} N(X)$ is a 2-sphere.

Note that $\chi\left(\partial_{0} N(X)\right)=\chi(\partial N(X))=2 \chi(N(X))=2 \chi(X)$. We prove Lemma 11.2 by computing that in all four cases the number of components of $\partial_{0} N(X)$ is $\chi(X)$; hence each must be a 2 -sphere. We compute $\chi(X)$ easily as follows.

Lemma 11.3. $\chi(X)=\frac{\Delta n_{1} n_{2}}{2}-n_{1}-n_{2}$.

Proof. First note that

$$
\begin{aligned}
\chi\left(F_{1} \cup F_{2}\right) & =\chi\left(F_{1}\right)+\chi\left(F_{2}\right)-\chi\left(F_{1} \cap F_{2}\right) \\
& =-n_{1}-n_{2}-\frac{\Delta n_{1} n_{2}}{2} .
\end{aligned}
$$




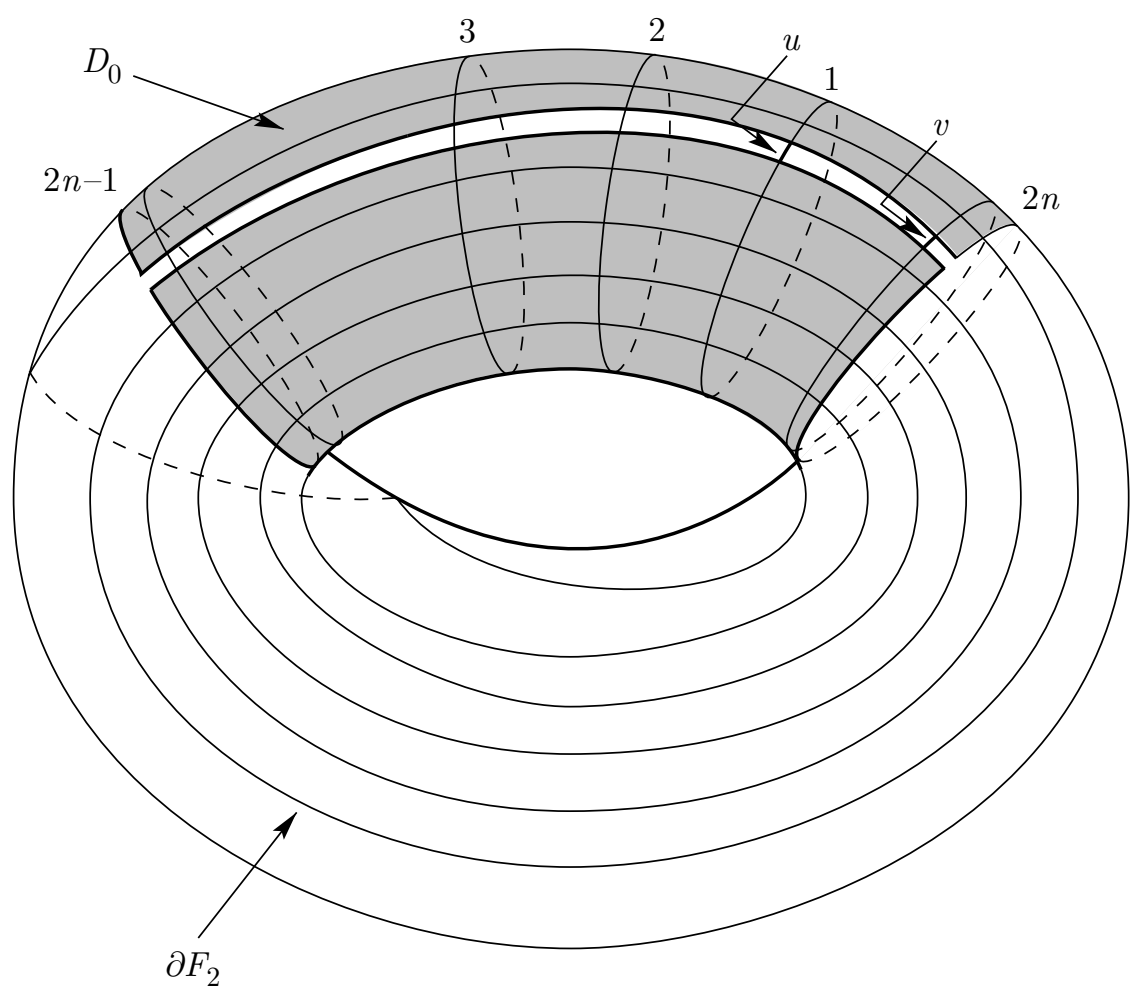

FiguRe 76

Also, $T \cap\left(F_{1} \cup F_{2}\right)=\partial F_{1} \cup \partial F_{2}$, and $\chi\left(\partial F_{1} \cup \partial F_{2}\right)=-\Delta n_{1} n_{2}$. Therefore

$$
\begin{aligned}
\chi(X) & =\chi(T)+\chi\left(F_{1} \cup F_{2}\right)-\chi\left(T \cap\left(F_{1} \cup F_{2}\right)\right) \\
& =\frac{\Delta n_{1} n_{2}}{2}-n_{1}-n_{2} \cdot \square
\end{aligned}
$$
12 .

Thus for $P(6), \chi(X)=8$; for $P(7), \chi(X)=4$; and for $P(8)_{1}$ and $P(8)_{2}, \chi(X)=$

We count the components of $\partial_{0} N(X)$ as follows. The $\operatorname{arcs} F_{1} \cap F_{2}$ decompose the surfaces $F_{1}, F_{2}$ into faces. Each of $F_{1}$ and $F_{2}$ is locally 2-sided in $N(X)$, so $\partial_{0} N(X)$ contains two copies $f^{+}, f^{-}$of each such face $f$. The number of components of the union of these $f^{ \pm}$'s is equal to the number of components of $\partial_{0} N(X)$ (which is obtained from this union by adding the 2-cells into which $T$ is divided by $\partial F_{1} \cup \partial F_{2}$ ).

Lemma 11.4. For $P(6), P(7), P(8)_{1}$, and $P(8)_{2}$, the number of components of $\partial_{0} N(X)$ is equal to $\chi(X)$.

Proof. We do $P(8)_{1}$ and $P(7)$ as examples, leaving the other similar verifications to the reader. The case of $P(7)$ is a little different from the others inasmuch as here one of the surfaces $\left(F_{2}\right)$ does not separate $N(X)$.

$P(8)_{1}$. Shade the faces in $F_{1}$ and $F_{2}$ alternately black and white as shown in Figure 78. (For convenience, we shall think of the surface shown in Figure 78(i) as $F_{1}$, and that shown in Figure 78(ii) as $F_{2}$. This differs from the notation of the last paragraph of Section 6.) Then the components of $\partial_{0} N(X)$ fall into four 


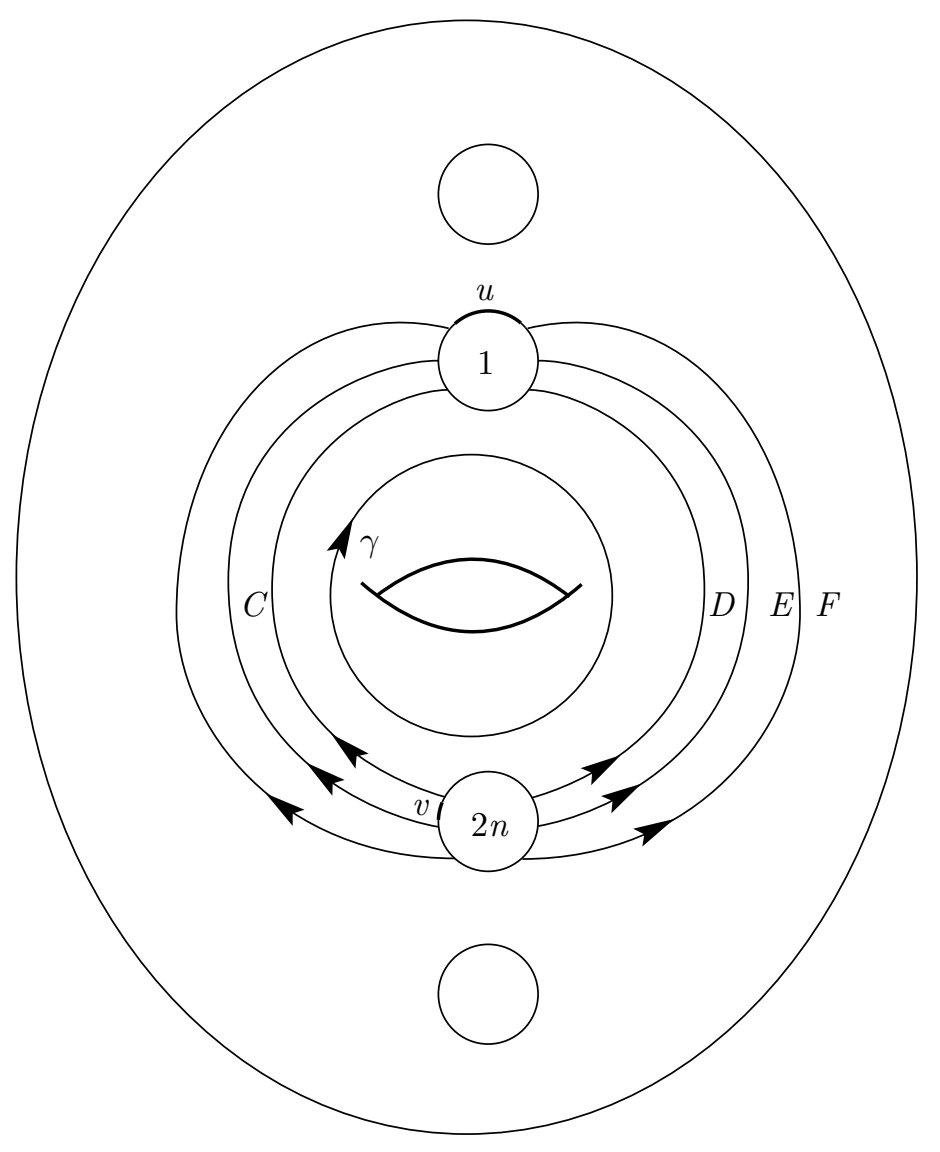

FiguRE 77

classes, defined in the obvious way: black/black, black/white, white/black, and white/white. The components in each of these classes are the unions of the following sets of faces, where we use subscripts to indicate which surface the given face lies in:

$$
\begin{array}{ll}
\text { black/black: } & A B_{1}, B G_{2}, G H_{1}, H A_{2} ; \\
& C D_{1}, D E_{2}, E F_{1}, F C_{2} ; \\
& W X_{1}, X V_{2}, V U_{1}, U W_{2} ; \\
& Y Z_{1}, Z T_{2}, T S_{1}, S Y_{2} ; \\
\text { black/white: } & A B_{1}, B E T_{2}, E F_{1}, T S_{1}, F A S_{2} ; \\
& C D_{1}, D W G_{2}, W X_{1}, G H_{1}, X H C_{2} ; \\
& U V_{1}, V Z_{2}, Z Y_{1}, Y U_{2} \\
\text { white/black: } & W U_{2}, U T H_{1}, T Z_{2}, H A_{2}, Z W A_{1} ; \\
& Y S_{2}, S V D_{1}, V X_{2}, D E_{2}, X Y E_{1} ; \\
& C F_{2}, F G_{1}, G B_{2}, B C_{1} ; \\
\text { white/white: } & B C_{1}, C H X_{2}, H U T_{1}, X E Y_{1}, U Y_{2}, T B E_{2} ; \\
& F G_{1}, G D W_{2}, D S V_{1}, W Z A_{1}, S A F_{2}, V Z_{2} .
\end{array}
$$

This gives a total of 12 , as claimed. 
C. McA. GORDON

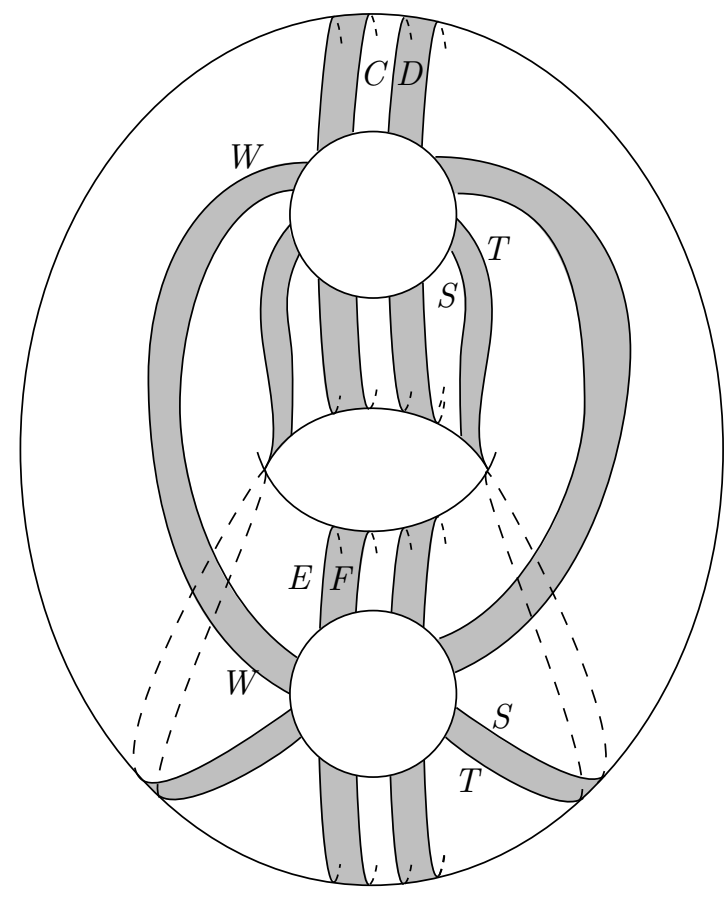

(i)

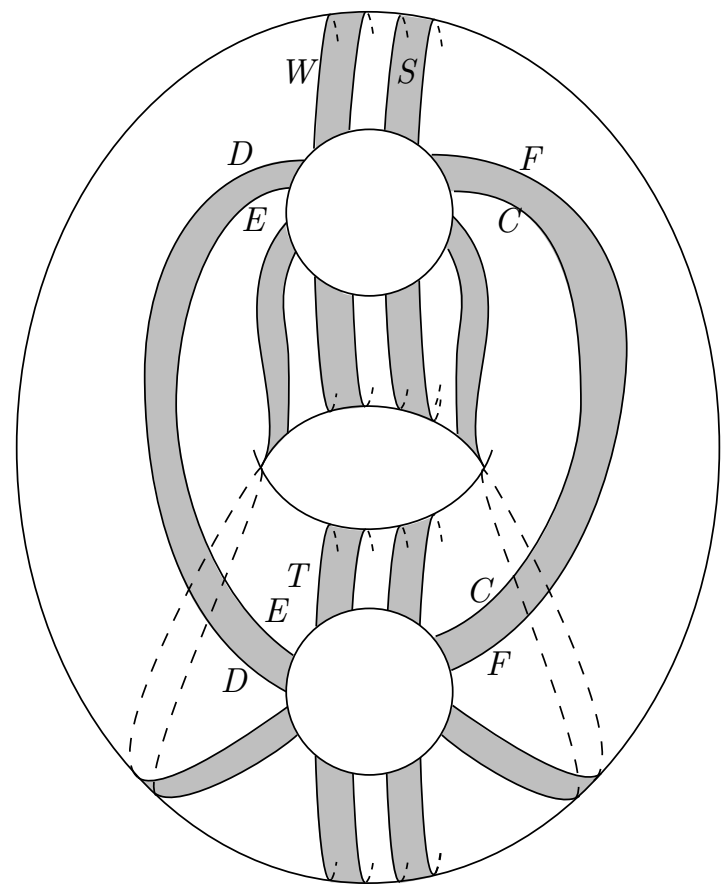

(ii)

Figure 78 


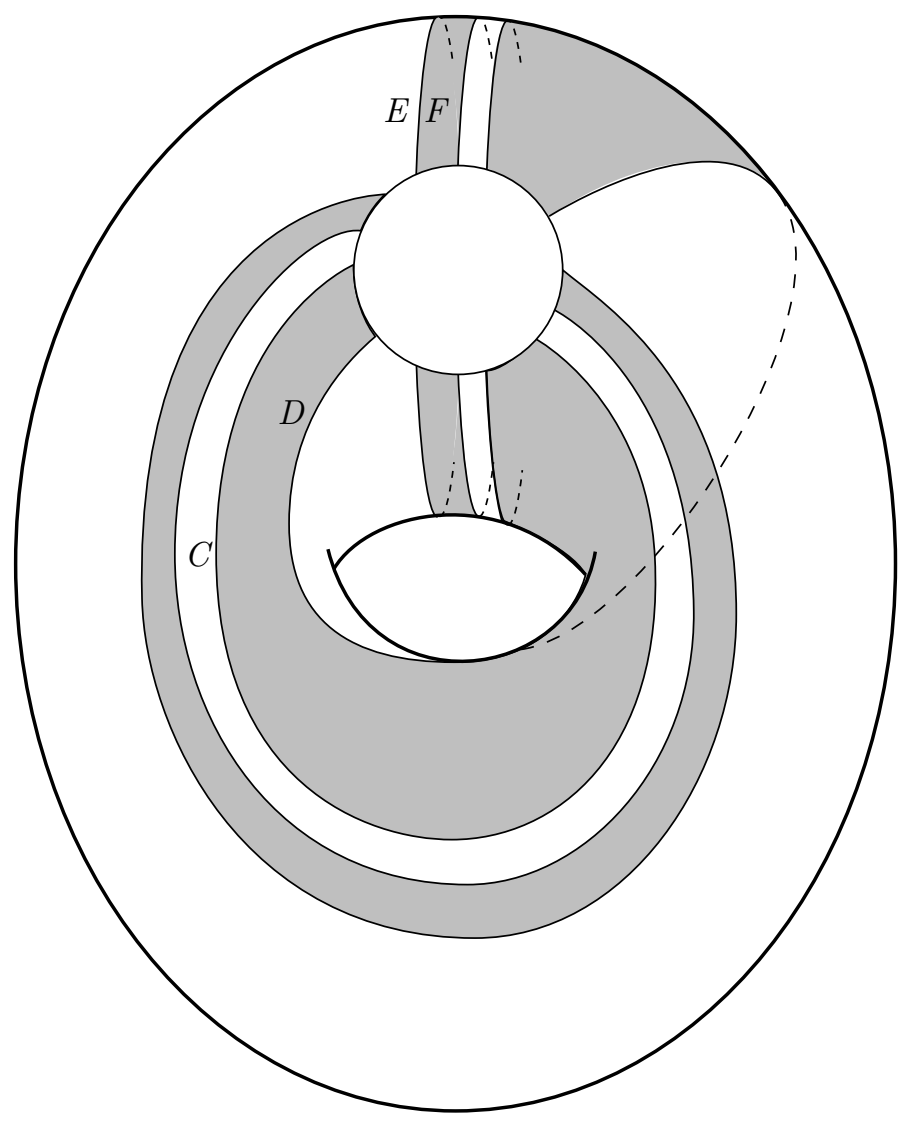

FigURE 79

$P(7)$. Since $F_{1}$ does separate $N(X)$, we can shade the faces on $F_{2}$ alternately black and white as shown in Figure 79. Although $F_{2}$ does not separate $N(X)$, it is of course locally 2 -sided. We designate the two sides + and - , and if $f$ is a face on $F_{2}$ then we denote by $f^{ \pm}$the push-off of $f$ in the appropriate direction. Then (referring also to Figure 35(i)) the components of $\partial_{0} N(X)$ are the unions of the following sets of faces, where we first list those involving black faces of $F_{2}$, and second those involving white faces of $F_{2}$ :

black: $A B_{2}^{+}, B F_{1}, F E_{2}^{-}, E A_{1}$;

$$
C D G_{2}^{+}, G D E B_{1}, C G_{1}, D G C_{2}^{-}, E F_{2}^{+}, B A_{2}^{-}, F C D A_{1} \text {. }
$$

white: $B C_{2}^{+}, C G_{1}, G F_{2}^{-}, F B_{1}$;

$$
D E A_{2}^{+}, E B G D_{1}, A E_{1}, E A D_{2}^{-}, B C_{2}^{-}, F G_{2}^{+}, A D C F_{1} \text {. }
$$

This gives a total of four components, as claimed.

Proof of Proposition 1.5. Suppose that $(M, T)$ is not cabled, and let $\left(F_{\alpha}, \partial F_{\alpha}\right) \subset$ $(M, T)$ be an essential punctured torus with boundary slope $r_{\alpha}, \alpha=1,2$, such that $\Delta\left(r_{1}, r_{2}\right) \geq 6$. After the work in the previous sections we know that $\left(F_{1}, F_{2} ; F_{1} \cap F_{2}\right)$ has to be given by one of the four identification patterns $P(6), P(7), P(8)_{1}$, or $P(8)_{2}$. Let $N$ be a regular neighborhood of $F_{1} \cup F_{2} \cup T$ in $M$. Then $N$ is homeomorphic 
to the appropriate $N(X)$ described above. By Lemma 11.2, $\partial N-T$ consists of 2 -spheres. Since $M$ is irreducible, $M=N \cup 3$-balls. Hence $\left(M ; F_{1}, F_{2}\right)$ is uniquely determined, up to homeomorphism, in each of the four cases. It follows that these must correspond to the examples described in Section 1, with $M=W(2), W(-5 / 2)$, $W(1)$, and $W(-5)$.

Remark. The identification patterns $P(8)_{1}$ and $P(8)_{2}$ are distinguishable by the fact that for $P(8)_{1}, d=1$, whilst for $P(8)_{2}, d=3$. Also, with suitable parametrizations, the slopes $r, s \in \mathcal{T}(M, \partial M)$ with $\Delta(r, s)=8$ are $4,-4$ for $M=W(1)$, and $\frac{1}{2},-\frac{3}{2}$ for $M=W(-5)$. Hence the punctured tori in $W(1)$ correspond to $P(8)_{1}$, and those in $W(-5)$, to $P(8)_{2}$.

\section{Other SURFACES With NON-NEGATIVE EULER CHARACTERISTIC}

We have now proved Proposition 1.5. Before discussing the case when $(M, T)$ is cabled, we consider other surfaces of non-negative euler characteristic, for applications to Theorems 1.2, 1.3, and 1.4. In this section we shall prove certain analogs of Proposition 1.5 in these cases.

Let $\mathcal{S}(M, T), \mathcal{D}(M, T), \mathcal{A}(M, T)$ denote the set of boundary slopes on $T$ of essential surface $F$ in $M$, with $F \cap T \neq \emptyset$, such that $\widehat{F}$ is homeomorphic to $S^{2}, D^{2}$, or the annulus $A^{2}$, respectively.

Proposition 12.1. If $r \in \mathcal{S}(M, T)$ and $s \in \mathcal{T}(M, T)$ then either $\Delta(r, s) \leq 5$ or $(M, T)$ is cabled.

Proof. This follows from [GLi, Proposition 6.1].

For $M$ hyperbolic with $\partial M=T$, this has recently been improved to $\Delta(r, s) \leq 4$ by Boyer and Zhang [BZ2], and subsequently to $\Delta(r, s) \leq 3$ by $\mathrm{Oh}[\mathrm{O}]$ and by Wu [Wu2].

Proposition 12.2. If $r \in \mathcal{S}(M, T) \cup \mathcal{T}(M, T)$ and $s \in \mathcal{D}(M, T) \cup \mathcal{A}(M, T)$, then either $\Delta(r, s) \leq 5$ or $(M, T)$ is cabled.

Proof. Let $F_{\alpha}$ be an essential surface in $M$, such that $F_{\alpha} \cap T \neq \emptyset$, with boundary slope $r_{\alpha}$ on $T, \alpha=1,2$, and such that $\widehat{F}_{1} \cong S^{2}$ or $T^{2}$ and $\widehat{F}_{2} \cong D^{2}$ or $A^{2}$. By considering the arcs of $F_{1} \cap F_{2}$ we get graphs $\Gamma_{\alpha} \subset \widehat{F}_{\alpha}, \alpha=1,2$. If $\widehat{F}_{1} \cong S^{2}$, then we can formally add a handle to $\widehat{F}_{1}$ and regard $\Gamma_{1}$ as a graph in $T^{2}$. Similarly, if $\widehat{F}_{2} \cong D^{2}$, we can remove a small open disk from $\widehat{F}_{2}$ and regard $\Gamma_{2}$ as a graph in $A^{2}$. So without loss of generality we assume that $\Gamma_{1} \subset T^{2}$ and $\Gamma_{2} \subset A^{2}$.

Suppose that $\Delta=\Delta\left(r_{1}, r_{2}\right) \geq 6$. By Lemmas 3.1 and 3.2, either $\Gamma_{1}$ has $n_{2}+1$ parallel edges, or $\Delta=6$ and each parallelism class of edges in $\Gamma_{1}$ has exactly $n_{2}$ members.

In the first case, adopting the notation used in the proof of Lemma 4.2, either $C_{\theta}(\boldsymbol{A})$ or $C_{\theta}(\boldsymbol{B})$ bounds a disk in the annulus $\widehat{F}_{2}$, or $A_{1}$ and $B_{1}$ are parallel on $F_{2}$. By Lemmas 2.1 and 2.3, we conclude that $(M, T)$ is cabled.

In the second case, by identifying the two boundary components of $\widehat{F}_{2} \cong A^{2}$, we can regard both $\Gamma_{1}$ and $\Gamma_{2}$ as graphs in $T^{2}$. Now the arguments of Sections 4-11 show that, unless $(M, T)$ is cabled, the only possibility for the pair $\Gamma_{1}, \Gamma_{2}$ is given by the pattern $P(6)$ illustrated in Figure 28. But neither graph there is contained in an annulus.

Proposition 12.3. If $r, s \in \mathcal{D}(M, T) \cup \mathcal{A}(M, T)$, then either 


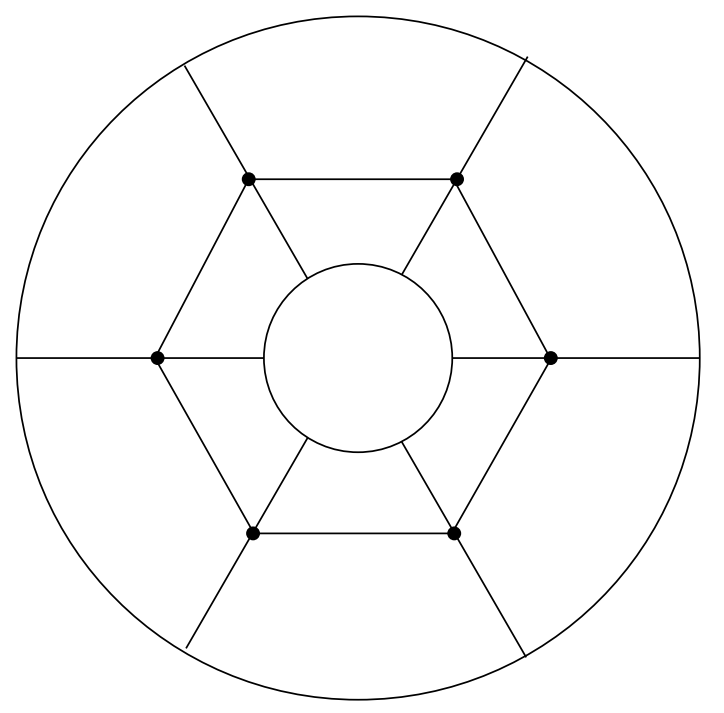

FiguRe 80

(1) $\Delta(r, s) \leq 5$; or

(2) $(M, T)$ is cabled; or

(3) $M$ contains an essential annulus having exactly one boundary component on $T$, with slope $r_{0}$ where $\Delta\left(r_{0}, r\right)=\Delta\left(r_{0}, s\right)=1$; or

(4) $M$ is homeomorphic to $T \times I$.

Proof. Let $F_{1}, F_{2}$ be essential surfaces in $M$ as above such that $\widehat{F}_{\alpha} \cong D^{2}$ or $A^{2}$, $\alpha=1,2$. The arcs of $F_{1} \cap F_{2}$ with at least one endpoint on $T$ give graphs $\Gamma_{1}, \Gamma_{2}$ in $\widehat{F}_{1}, \widehat{F}_{2}$ respectively. As in the proof of Proposition 12.2 , we may assume that $\widehat{F}_{1}$ and $\widehat{F}_{2}$ are annuli.

Suppose that $\Delta=\Delta\left(r_{1}, r_{2}\right) \geq 6$. Then by Lemma 3.3, $\Gamma_{1}$ has either $n_{2}+1$ parallel internal edges or $2 n_{2}$ parallel boundary edges.

In the first case we conclude that $(M, T)$ is cabled exactly as in the proof of Proposition 12.2.

In the second case, let $A_{1}, \ldots, A_{n_{2}}, B_{1}, \ldots, B_{n_{2}}$ be the corresponding parallel arcs of $F_{1} \cap F_{2}$ on $F_{1}$, numbered in order so that (without loss of generality) $A_{i}$ and $B_{i}$ each has label $i$ at its endpoint on $\partial F_{1} \cap T, 1 \leq i \leq n_{2}$. If, for some $i$, both $A_{i}$ and $B_{i}$ go to the same boundary component of the annulus $\widehat{F}_{2}$, then we get either conclusion (3) or conclusion (4), as in [CGLS, Lemmas 2.5.4 and 2.5.5]. So we may suppose that in $\Gamma_{2}$, from each vertex there are boundary edges going to both boundary components of $\widehat{F}_{2}$. Thus the reduced graph $\bar{\Gamma}_{2}$ is a subgraph of a graph of the form illustrated in Figure 80.

If $\Gamma_{2}$ has $n_{1}+1$ parallel internal edges, then we conclude as before that $(M, T)$ is cabled. Also, if $\Gamma_{2}$ has $2 n_{1}+1$ parallel boundary edges, then two of these must correspond to edges in $\Gamma_{1}$ joining some vertex to the same boundary component of $\widehat{F}_{1}$, so again we get conclusion (3) or (4) as in [CGLS, Lemmas 2.5.4 and 2.5.5]. It follows that $\bar{\Gamma}_{2}$ is as illustrated in Figure $80, \Delta=6$, each parallelism class of internal edges of $\Gamma_{2}$ has exactly $n_{1}$ members, and each parallelism class of boundary edges 


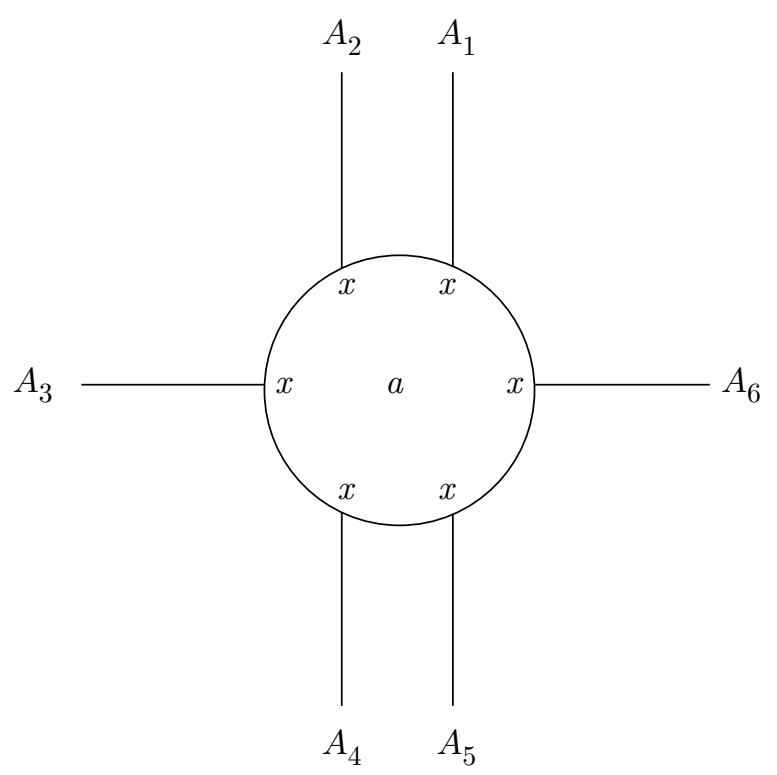

FIGURE 81

has exactly $2 n_{1}$ members. Since the argument is symmetrical, the corresponding statement also holds for $\Gamma_{1}$.

Now let $a$ be a component of $\partial F_{1} \cap T$, and let $A_{1}, A_{2}, \ldots, A_{6}$ be the arcs of $F_{1} \cap F_{2}$ with label $x$ at $a$, numbered cyclically around $a$ so that $A_{1}, A_{2}, A_{4}$, and $A_{5}$ are boundary edges and $A_{3}, A_{6}$ are internal edges (see Figure 81). On $F_{2}, A_{1}$ and $A_{2}$ must go to distinct boundary components of $\widehat{F}_{2}$ (otherwise we get conclusion (3) or (4) by [CGLS, Lemma 2.5.4]), and similarly for $A_{4}$ and $A_{5}$. But since $d=1$, $A_{1}, A_{2}, \ldots, A_{6}$ occur in the same cyclic order around $x$ in $F_{2}$. This is clearly a contradiction.

\section{CABled MANifoldS}

Let $(M, T)$ be cabled, so $M=M^{\prime} \cup C$, where $C$ is a $(p, q)$-cable space, $\partial C=$ $T \amalg T^{\prime}$, and $M^{\prime} \cap C=T^{\prime}$. Let $F$ be an essential surface in $M$, isotoped so as to minimize the number of components of $F \cap T^{\prime}$. Then $F \cap C$ and $F^{\prime}=F \cap M^{\prime}$ are essential in $C$ and $M^{\prime}$ respectively. Now suppose that $F \cap T \neq \emptyset$ and that $F \cap C$ is planar. The essential planar surfaces in a cable space are described in [GLi, Lemma 3.1]. In particular, adopting the terminology of that lemma, there are four possibilities for $F \cap C$ :

an annulus of type (1);

a number of annuli of type (3), possibly together with some annuli of type (2);

a number of parallel copies of a surface of type (4);

a number of parallel copies of a surface of type (5).

We shall say that $F$ is of type (1), (3), (4), or (5) respectively.

Throughout this section, we refer the reader to [GLi, Lemma 3.1] for the descriptions of the boundary slopes of planar surfaces in cable spaces. 
Lemma 13.1. Let $r_{\alpha}, r_{\alpha}^{\prime}$ be the inner and outer boundary slopes of a planar surface of type (4) in a $(p, q)$-cable space, $\alpha=1,2$, with $r_{1} \neq r_{2}$.

(i) There exists a slope $r_{0}$ on $T$ such that $\Delta\left(r_{0}, r_{\alpha}\right)=1, \alpha=1,2$.

(ii) There exists a slope $r_{0}^{\prime}$ on $T^{\prime}$ such that $\Delta\left(r_{0}^{\prime}, r_{\alpha}^{\prime}\right)=1, \alpha=1,2$, if and only if $q=2$ and $\Delta\left(r_{1}, r_{2}\right)=1$.

Proof. (i) With respect to the standard cable space coordinates,

$$
r_{\alpha}=\left(1+k_{\alpha} p q\right) / k_{\alpha},
$$

$\alpha=1,2$, for some integers $k_{1}, k_{2}$. Then $r_{0}=p q$ (the slope of an ordinary fibre in the Seifert fibre space structure) satisfies $\Delta\left(r_{0}, r_{\alpha}\right)=1, \alpha=1,2$.

(ii) With respect to the standard cable space coordinates, $r_{\alpha}^{\prime}=\left(1+k_{\alpha} p q\right) / k_{\alpha} q^{2}$. Suppose $r_{0}^{\prime}=x / y$ satisfies $\Delta\left(r_{0}^{\prime}, r_{\alpha}^{\prime}\right)=1, \alpha=1,2$. Then

$$
\left(1+k_{\alpha} p q\right) y-k_{\alpha} q^{2} x= \pm 1
$$

that is

$$
y+k_{\alpha} q(p y-q x)= \pm 1, \quad \alpha=1,2 .
$$

Subtracting, we obtain

$$
\left(k_{1}-k_{2}\right) q(p y-q x)=0 \text { or } \pm 2 .
$$

The first case gives $p y-q x=0$, and (hence) $y= \pm 1$, contradicting the fact that $(p, q)=1$. The second case gives $q=2$ and $\left|k_{1}-k_{2}\right|=\Delta\left(r_{1}, r_{2}\right)=1$. Conversely, if $q=2$ and $\left|k_{1}-k_{2}\right|=1$ then one easily verifies that an $r_{0}^{\prime}$ with the desired property exists.

Let $F_{1}, F_{2}$ be essential surfaces in $M$ as above with boundary slopes $r_{1}, r_{2}$ on $T$, and let $\Delta=\Delta\left(r_{1}, r_{2}\right)$. The following lemma is an immediate consequence of the description of the boundary slopes in [GLi, Lemma 3.1].

Lemma 13.2. Let $M, F_{1}, F_{2}$ be as above.

(a) If $F_{\alpha}$ is of type (1) or (3), $\alpha=1,2$, then $r_{1}=r_{2}$.

(b) If $F_{1}$ is of type (1) or (3) and $F_{2}$ is of type (4), then $\Delta=1$.

(c) If $F_{1}$ is of type (1) or (3) and $F_{2}$ is of type (5), then $\Delta=q$.

(d) If $F_{1}$ is of type (4) and $F_{2}$ is of type (5), then $(\Delta, q)=1$.

In the next two lemmas, $F_{1}$ and $F_{2}$ are essential surfaces in $M=M^{\prime} \cup C$, with boundary slopes $r_{1}, r_{2}$ on $T$ and $r_{1}^{\prime}, r_{2}^{\prime}$ on $T^{\prime}$. We assume $r_{1} \neq r_{2}$ and write $\Delta=$ $\Delta\left(r_{1}, r_{2}\right), \Delta^{\prime}=\Delta\left(r_{1}^{\prime}, r_{2}^{\prime}\right)$. We also assume that $\left(M^{\prime}, T^{\prime}\right)$ is cabled, the corresponding cable space being $C^{\prime}$.

The calculation in the proof of Lemma 13.1(ii) is very similar to that given in [GLi, p.137], where it is used to prove the next lemma.

Lemma 13.3. Let $M, F_{1}, F_{2}$ be as above. If $\left(M^{\prime}, T^{\prime}\right)$ is cabled and $F_{1}, F_{2}, F_{1}^{\prime}, F_{2}^{\prime}$ are of type (4), then $q=2$ and $\Delta=1$.

Proof. This follows from Lemma 13.1.

Lemma 13.4. Let $M, F_{1}, F_{2}$ be as above, where $F_{1}$ and $F_{2}$ are of type (4). Suppose that $\left(M^{\prime}, T^{\prime}\right)$ is $(1,2)$-cabled.

(a) If $F_{1}^{\prime}$ is of type (4) and $F_{2}^{\prime}$ is of type (5), then $\Delta=1$. 
(b) If $F_{1}^{\prime}$ and $F_{2}^{\prime}$ are of type (5), then either $q=2$ and the identification of $C$ and $C^{\prime}$ along $T^{\prime}$ preserves the fibres of the Seifert fibrations of $C$ and $C^{\prime}$, or $\Delta \leq 2$.

Proof. With respect to the standard cable coordinates on $C, r_{\alpha}^{\prime}=\left(1+k_{\alpha} p q\right) / k_{\alpha} q^{2}$, $\alpha=1,2$.

The identification of the outer boundary of $C$ with the inner boundary of $C^{\prime}$ is given by a matrix $\left[\begin{array}{ll}x & y \\ z & w\end{array}\right] \in G L(2, \mathbb{Z})$.

(a) With respect to the standard cable coordinates on $C^{\prime}$,

$$
\begin{aligned}
& r_{1}^{\prime}=(1+2 k) / k, \\
& r_{2}^{\prime}=(2+2 m) / m,
\end{aligned}
$$

for some integers $k$ and $m$.

Applying the identification matrix to the $C$-coordinates of $r_{1}^{\prime}$ and $r_{2}^{\prime}$ gives the four equations

$$
\begin{aligned}
x\left(1+k_{1} p q\right)+y k_{1} q^{2} & =\varepsilon_{1}(1+2 k), \\
z\left(1+k_{1} p q\right)+w k_{1} q^{2} & =\varepsilon_{1} k, \\
x\left(1+k_{2} p q\right)+y k_{2} q^{2} & =\varepsilon_{2}(2+2 m), \\
z\left(1+k_{2} p q\right)+w k_{2} q^{2} & =\varepsilon_{2} m,
\end{aligned}
$$

where $\varepsilon_{\alpha}= \pm 1, \alpha=1,2$. Taking ((i)-2(ii))-((iii)-2(iv)), we get

$$
\left(k_{1}-k_{2}\right) q(p(x-2 z)+q(y-2 w))=\varepsilon_{1}-2 \varepsilon_{2}=-1 \quad \text { or } \quad \pm 3 .
$$

Therefore $\left|k_{1}-k_{2}\right|=\Delta=1($ and $q=3)$.

(b) With respect to the standard cable coordinates on $C^{\prime}$,

$$
r_{\alpha}^{\prime}=\left(2+2 m_{\alpha}\right) / m_{\alpha}, \quad \alpha=1,2,
$$

for some integer $m_{1}, m_{2}$.

Hence, as in (a) above, we get two equations

$$
\begin{aligned}
x\left(1+k_{\alpha} p q\right)+y k_{\alpha} q^{2} & =\varepsilon_{\alpha}\left(2+2 m_{\alpha}\right), \\
z\left(1+k_{\alpha} p q\right)+w k_{\alpha} q^{2} & =\varepsilon_{\alpha} m_{\alpha},
\end{aligned}
$$

for each $\alpha=1$, 2. Subtracting (i) $)_{2}$ from (i) $)_{1}$ and (ii) $)_{2}$ from (ii) $)_{1}$ gives

$$
\begin{aligned}
\left(k_{1}-k_{2}\right) q(x p+y q) & =2\left(\left(\varepsilon_{1}-\varepsilon_{2}\right)+\left(\varepsilon_{1} m_{1}-\varepsilon_{2} m_{2}\right)\right), \\
\left(k_{1}-k_{2}\right) q(z p+w q) & =\varepsilon_{1} m_{1}-\varepsilon_{2} m_{2} .
\end{aligned}
$$

If $\varepsilon_{1}=\varepsilon_{2}$, then these equations show that

$$
x p+y q=2(z p+w q) .
$$

Since $(x p+y q, z p+w q)=1$, this implies that

$$
z p+w q=\varepsilon, \quad x p+y q=2 \varepsilon, \text { where } \varepsilon= \pm 1 ;
$$

in other words,

$$
\left[\begin{array}{cc}
x & y \\
z & w
\end{array}\right]\left[\begin{array}{l}
p \\
q
\end{array}\right]=\left[\begin{array}{c}
2 \varepsilon \\
\varepsilon
\end{array}\right] .
$$


Since the slopes on $T^{\prime}$ of the fibres of $C$ and $C^{\prime}$ are $p / q$ and 2/1 respectively, this shows that the identification of the outer boundary of $C$ with the inner boundary of $C^{\prime}$ is fibre-preserving. Also, equation (vi) now gives

$$
\left|k_{1}-k_{2}\right| q=\left|m_{1}-m_{2}\right| \text {. }
$$

But $\left|k_{1}-k_{2}\right| q^{2}=\Delta^{\prime}=2\left|m_{1}-m_{2}\right|$. Therefore $q=2$.

If $\varepsilon_{1}=-\varepsilon_{2}$, then (v)-2(vi) gives an equation of the form

$$
\left(k_{1}-k_{2}\right) q X= \pm 4 \text {. }
$$

Hence $\Delta=\left|k_{1}-k_{2}\right| \leq 2$.

Lemma 13.5. Let $M$ be a Seifert fibre space with orbit surface a disk and two exceptional fibres of multiplicities $q_{1}, q_{2}$. Then $M$ contains exactly two essential surfaces, one a vertical annulus and the other horizontal, with boundary slopes $r_{0}$ and $r_{1}$, say. Moreover if $r_{1} \in \mathcal{S}(M, \partial M)$ then $\Delta\left(r_{0}, r_{1}\right)=1$, and if $r_{1} \in \mathcal{T}(M, \partial M)$ then $\left\{q_{1}, q_{2}, \Delta\left(r_{0}, r_{1}\right)\right\}=\{3,3,3\},\{2,4,4\}$, or $\{2,3,6\}$.

If $q_{1}=2$ and $q_{2}=3$ then $M$ is homeomorphic to the exterior of the trefoil knot, so the following corollary is immediate. It will be used in the proof of Proposition 13.7.

Corollary 13.6. Let $M$ be as in Lemma 13.5, and suppose $r, s \in \mathcal{S}(M, \partial M) \cup$ $\mathcal{T}(M, \partial M)$. Then either $\Delta(r, s) \leq 4$, or $M$ is homeomorphic to the exterior of the trefoil knot, $r($ say $) \in \mathcal{S}(M, \partial M), s \in \mathcal{T}(M, \partial M)$, and $\Delta(r, s)=6$.

Proof of Lemma 13.5. Note that $M=M^{\prime} \cup C$, where $C$ is a $\left(p_{1}, q_{1}\right)$-cable space and $M^{\prime}$ is a solid torus. Let $F$ be an essential surface in $M$, isotoped so as to intersect $M^{\prime}$ minimally. Then $F \cap C$ is an essential surface in $C$, and is therefore either a vertical annulus or horizontal. In the second case the boundary slope of $F \cap C$ on $\partial M^{\prime}$ must be that of a meridian disk of $M^{\prime}$. It then follows from [GLi, proof of Lemma 3.1] that $F \cap C$, and hence $F$, is uniquely determined.

If $r \in \mathcal{S}(M, \partial M)$, then $\Delta\left(r_{0}, r\right)=1$ by [GLi, Proposition 1.4].

If $r \in \mathcal{T}(M, \partial M)$, and $F$ is the punctured torus in $M$ with boundary slope $r$, then $\widehat{F}$ is a horizontal torus in $M(r)$. Note that $M(r)$ is a Seifert fibre space with orbit surface $S^{2}$ and (at most) 3 exceptional fibres of multiplicities $q_{1}, q_{2}$, and $q_{3}=\Delta\left(r_{0}, r\right) \geq 1$. Hence $\widehat{F}$ is a $k$-fold covering of $S^{2}$ branched over 3 points $x_{1}, x_{2}, x_{3}$ with branching indices $q_{1}, q_{2}, q_{3}$. Let $b_{i}$ be the number of lifts of $x_{i}$ in $\widehat{F}$, $i=1,2,3$. Then $k=b_{i} q_{i}, i=1,2,3$. Also,

$$
\begin{aligned}
0=\chi(\widehat{F}) & =k \chi\left(S^{2}-\left\{x_{1}, x_{2}, x_{3}\right\}\right)+b_{1}+b_{2}+b_{3} \\
& =-k+b_{1}+b_{2}+b_{3} \\
& =k\left(\frac{1}{q_{1}}+\frac{1}{q_{2}}+\frac{1}{q_{3}}-1\right) .
\end{aligned}
$$

Therefore $\frac{1}{q_{1}}+\frac{1}{q_{2}}+\frac{1}{q_{3}}=1$, giving the three euclidean triples listed.

Let $\mathcal{B}_{+}(M, T)$ denote the set of boundary slopes on $T$ of essential surfaces $F$ in $M$ with $\chi(\widehat{F}) \geq 0$, that is, $\mathcal{B}_{+}(M, T)=\mathcal{S}(M, T) \cup \mathcal{T}(M, T) \cup \mathcal{D}(M, T) \cup \mathcal{A}(M, T)$.

Proposition 13.7. Suppose that $(M, T)$ is cabled and that $\partial M$ is incompressible. If $r, s \in \mathcal{B}_{+}(M, T)$, then either

(1) $\Delta(r, s) \leq 4$; or 
(2) $M$ is homeomorphic to the exterior of the trefoil knot, $r($ say $) \in \mathcal{S}(M, T)$, $s \in \mathcal{T}(M, T)$, and $\Delta(r, s)=6$; or

(3) $M$ is a cable space, and $r, s \in \mathcal{D}(M, T) \cup \mathcal{A}(M, T)$; or

(4) $M$ is a Seifert fibre space with orbit surface an annulus and two exceptional fibres of multiplicity 2 , and $r, s \in \mathcal{A}(M, T)$.

Before proving this proposition, we observe the following corollary, in which we drop the assumption that $\partial M$ is incompressible. Note that Proposition 1.6 is an immediate consequence.

Corollary 13.8. If $(M, T)$ is cabled and $r, s \in \mathcal{B}_{+}(M, T)$ then either conclusion (1) or (2) of Proposition 13.7 holds, or $r, s \in \mathcal{D}(M, T) \cup \mathcal{A}(M, T)$ and $M$ contains an essential annulus with exactly one boundary component on $T$.

Proof. If $\partial M$ is incompressible, then the corollary follows immediately from Proposition 13.7 .

If $\partial M$ is compressible, let $W$ be a maximal compression body for $\partial M-T$ in $M$, and let $M_{0}=\overline{M-W}$. If $F$ is an essential surface in $M$ (such that $\partial F \cap T \neq \emptyset$ ), isotoped so as to minimize the number of components of $F \cap \partial M_{0}$, then $F \cap M_{0}$ and $F \cap W$ are essential. Hence each component of $F \cap W$ is an annulus with one boundary component on $\partial M_{0}$ and the other on $\partial M$. If $r, s \in \mathcal{B}_{+}(M, T)$ we can therefore apply Proposition 13.7 to the corresponding surfaces $F \cap M_{0}$ in $M_{0}$. If conclusion (1) or (2) holds for $M_{0}$, then it holds for $M$. If conclusion (3) or (4) holds for $M_{0}$, then there is an essential annulus in $M_{0}$ with one boundary component on $T$ and the other, $\gamma$, say, on $\partial M_{0}-T$. Since there is an annulus in $W$ with one boundary on $\partial M$ and the other equal to $\gamma$, we get an annulus in $M$ as described.

Proof of Proposition 13.7. Let $F_{1}, F_{2}$ be essential surfaces in $M=M^{\prime} \cup C$ as above, such that $\widehat{F}_{\alpha} \cong S^{2}, T^{2}, D^{2}$, or $A^{2}, \alpha=1,2$. Let $r_{\alpha}$ be the boundary slope of $F_{\alpha}$ on $T$, and $r_{\alpha}^{\prime}$ the boundary slope of $F_{\alpha}^{\prime}=F_{\alpha} \cap M^{\prime}$ on $T^{\prime}$ (if $F_{\alpha}^{\prime} \neq \emptyset$ ), $\alpha=1,2$. Let $\Delta=\Delta\left(r_{1}, r_{2}\right)$ and $\Delta^{\prime}=\Delta\left(r_{1}^{\prime}, r_{2}^{\prime}\right)$. We suppose $r_{1} \neq r_{2}$, therefore $r_{1}^{\prime} \neq r_{2}^{\prime}$ (see [GLi, proof of Lemma 3.1]).

If $\widehat{F}_{1} \cong \widehat{F}_{2} \cong S^{2}$, then $\Delta=1$ by [GLi, Proposition 1.4], so we need not consider this case.

First note that if some component of $F_{\alpha} \cap C$ is a punctured torus, then some component of $F_{\alpha}^{\prime}$ is a disk, and hence $M^{\prime}$ is a solid torus. Therefore $M$ is a Seifert fibre space over the disk with two exceptional fibres, and the result follows from Corollary 13.6. We may therefore assume that all components of $F_{1} \cap C$ and $F_{2} \cap C$ are planar.

If $F_{\alpha}$ is of type (5), then, by considering the euler characteristics of $F_{\alpha}, F_{\alpha} \cap C$, and $F_{\alpha}^{\prime}$, we easily conclude the following:

(i) the case $\widehat{F}_{\alpha} \cong D^{2}$ is impossible;

(ii) if $\widehat{F}_{\alpha} \cong S^{2}$, then $F_{\alpha}$ is a disk, contradicting the incompressibility of $T$;

(iii) if $\widehat{F}_{\alpha} \cong T^{2}$ or $A^{2}$, then $q=2$ and $F_{\alpha}^{\prime}$ consists of annuli.

In case (iii), suppose that $\widehat{F}_{\alpha} \cong A^{2}$. Then some component $E_{\alpha}$, say, of $F_{\alpha}^{\prime}$ has exactly one boundary component on $T^{\prime}$. If $\widehat{F}_{\beta} \cong T^{2}$ and $F_{\beta}^{\prime} \neq \emptyset$, then by considering $E_{\alpha} \cap F_{\beta}^{\prime}$ we see that $F_{\beta}^{\prime}$ is boundary-compressible, and hence compressible, a contradiction. If $\widehat{F}_{\beta} \cong A^{2}$, then $F_{\beta}^{\prime}$ has a component $E_{\beta}$ with exactly one boundary component on $T^{\prime}$. By hypothesis, the annuli $E_{1}$ and $E_{2}$ have distinct boundary 
slopes on $T^{\prime}$, and hence it easily follows, using the irreducibility of $M$ and the incompressibility of $\partial M$, that $M^{\prime} \cong T^{\prime} \times I$ (compare [CGLS, Lemma 2.5.3]). Thus $M$ is a cable space. It now readily follows that we must have either conclusion (3) or conclusion (1).

After Lemma 13.2(a), (b), and (c), then, we may assume that we are in one of the following three cases, and that if $F_{\alpha}$ is of type (5) then $\widehat{F}_{\alpha} \cong T^{2}$ :

I. $F_{1}$ and $F_{2}$ of type (4);

II. $F_{\alpha}$ of type (4) and $F_{\beta}$ of type (5);

III. $F_{1}$ and $F_{2}$ of type (5).

First we dispose of Case III. Here $q=2, F_{1}^{\prime}$ and $F_{2}^{\prime}$ consist of annuli with their boundaries on $T^{\prime}$, and $\Delta=4 \Delta^{\prime}$. By [GLi, p.139], $\Delta^{\prime}=1$, and hence $\Delta=4$.

To treat Cases I and II, we proceed by induction on the cable length of $(M, T)$, which is defined in the obvious way as follows: if $(M, T)$ is not cabled it has cable length 0 , and if $(M, T)$ is a cabling of $\left(M^{\prime}, T^{\prime}\right)$ then cable length $(M, T)=$ cable length $\left(M^{\prime}, T^{\prime}\right)+1$.

Let us consider Case II. Here $q=2, F_{\beta}^{\prime}$ consists of annuli with their boundaries on $T^{\prime}$, and $\Delta^{\prime}=\Delta$. Let $E$ be a component of $F_{\beta}^{\prime}$ and consider $E \cap F_{\alpha}^{\prime}$. If $2 \Delta^{\prime}>6$ then some pair of arcs of intersection must be parallel on $F_{\alpha}^{\prime}$. (If $\widehat{F}_{\alpha}^{\prime} \cong$ $T^{2}$, this follows from Lemma 3.1. The case $\widehat{F}_{\alpha}^{\prime} \cong S^{2}$ also follows formally from that lemma by adding a handle. Similarly, the cases $\widehat{F}_{\alpha}^{\prime} \cong A^{2}$ or $D^{2}$ follow by embedding $A^{2}$ or $D^{2}$ in $T^{2}$, where, in the case of $A^{2}$, the embedding is chosen to be essential.) Since all arcs of intersection are necessarily parallel on $E$, we conclude from Lemma 2.1 that either $\Delta=\Delta^{\prime} \leq 3$ or $\left(M^{\prime}, T^{\prime}\right)$ is cabled. In the second case we may assume by induction, since $r_{\beta}^{\prime} \in \mathcal{S}\left(M^{\prime}, T^{\prime}\right)$, that either $\Delta^{\prime} \leq 4$ or $\Delta^{\prime}=6$. But by Lemma 13.2(d), $\Delta$ is odd. Hence $\Delta \leq 3$.

Finally we consider Case I. Here $\Delta^{\prime}=q^{2} \Delta$. By Propositions 1.5, 12.1, 12.2, and 12.3 , either

(i) $\Delta^{\prime} \leq 8$; or

(ii) $\left(M^{\prime}, T^{\prime}\right)$ is cabled; or

(iii) there exists a slope $r_{0}^{\prime}$ on $T^{\prime}$ such that $\Delta\left(r_{0}^{\prime}, r_{\alpha}^{\prime}\right)=1, \alpha=1,2$; or

(iv) $M^{\prime}$ is homeomorphic to $T^{\prime} \times I$.

In case (i), we get $\Delta \leq 2$.

In case (iii), we get $\Delta=1$ by Lemma 13.1(ii).

In case (iv), $M$ is a cable space and we get conclusion (3).

In case (ii), we assume by induction that Proposition 13.7 holds for $\left(M^{\prime}, T^{\prime}\right)$. Thus either $\Delta^{\prime} \leq 6$ (in which case $\Delta=1$ ), or conclusion (3) or (4) holds for $\left(M^{\prime}, T^{\prime}\right)$.

First suppose that conclusion (3) holds. Then $M=C \cup C^{\prime}$, where $C^{\prime}$ is a $\left(p^{\prime}, q^{\prime}\right)$-cable space, say, and $r_{1}^{\prime}, r_{2}^{\prime} \in \mathcal{D}\left(C^{\prime}, T^{\prime}\right) \cup \mathcal{A}\left(C^{\prime}, T^{\prime}\right)$. We consider the various possibilities for $F_{1}^{\prime}$ and $F_{2}^{\prime}$, which must be of type (3), (4), or (5). Recall that if $F_{\alpha}^{\prime}$ is of type (5) then $q^{\prime}=2$.

If $F_{\alpha}^{\prime}$ is of type (3), then $\Delta^{\prime} \leq 2$ by Lemma 13.2 (b) and (c), a contradiction.

If $F_{1}^{\prime}$ and $F_{2}^{\prime}$ are of type (4), then $\Delta=1$ by Lemma 13.3 .

If $F_{\alpha}^{\prime}$ is of type (4) and $F_{\beta}^{\prime}$ is of type (5), then $\Delta=1$ by Lemma 13.4(a).

If $F_{1}^{\prime}$ and $F_{2}^{\prime}$ are of type (5), then, by Lemma 13.4(b), either $\Delta \leq 2$ or conclusion (4) of Proposition 13.7 holds. 
Finally, if conclusion (4) holds for $\left(M^{\prime}, T^{\prime}\right)$, then note that (by induction) we may assume that this case only arises when $F_{1}^{\prime}$ and $F_{2}^{\prime}$ are both of type (4). But then $\Delta=1$ by Lemma 13.3 .

An example where $(M, T)$ is cabled and has $r, s \in \mathcal{T}(M, T)$ with $\Delta(r, s)=4$ is the following. Let $M^{\prime}$ be the Seifert fibre space over the disk with two exceptional fibres of multiplicity 2. Equivalently, $M^{\prime}$ is the exterior of the $(1,2)$-cable of a core of $S^{1} \times S^{2}$. Then $M^{\prime}$ contains two essential annuli (one vertical, the other horizontal) with boundary slopes $r_{1}^{\prime}, r_{2}^{\prime}$ such that $\Delta\left(r_{1}^{\prime}, r_{2}^{\prime}\right)=1$. (In fact, $M^{\prime}$ is the only example of a manifold that contains two essential annuli with distinct boundary slopes; see [GLi, pp.138-139].) Now if we let $(M, \partial M)$ be the $(1,2)$-cabling of $\left(M^{\prime}, \partial M^{\prime}\right)$, it is easy to construct essential punctured tori $F_{1}, F_{2}$ in $M$, of type (5), with boundary slopes $r_{1}, r_{2}$ such that $\Delta\left(r_{1}, r_{2}\right)=4$.

\section{FinAl PROOFS}

First we combine the results of Sections 12 and 13 on $\mathcal{B}_{+}(M, T)$.

For spheres, we recall the following, which is proved in [GLu], and also [BZ1].

Theorem 14.1. If $r, s \in \mathcal{S}(M, T)$ then $\Delta(r, s) \leq 1$.

Actually, the bound $\Delta(r, s) \leq 4$, obtained in [GLi, Theorem 1.1], would suffice for our present purposes.

For tori, we have Theorem 1.1, which follows from Propositions 1.5 and 1.6. The former was proved in Sections 4-11, whilst the latter is a consequence of Corollary 13.8.

The next three theorems follow from Propositions 12.1, 12.2, and 12.3 respectively, together with Corollary 13.8.

Theorem 14.2. If $r \in \mathcal{S}(M, T)$ and $s \in \mathcal{T}(M, T)$, then either $\Delta(r, s) \leq 5$, or $\Delta(r, s)=6$ and $M$ is homeomorphic to the exterior of the trefoil knot.

Theorem 14.3. If $r \in \mathcal{S}(M, T) \cup \mathcal{T}(M, T)$ and $s \in \mathcal{D}(M, T) \cup \mathcal{A}(M, T)$, then $\Delta(r, s) \leq 5$.

Theorem 14.4. If $r, s \in \mathcal{D}(M, T) \cup \mathcal{A}(M, T)$ then either $\Delta(r, s) \leq 5$ or $M$ contains an essential annulus with exactly one boundary component on $T$.

In fact, if $r, s \in \mathcal{D}(M, T)$, then the bound of 5 in Theorem 14.4 can be replaced by 1 [Wu1].

It is clear that other improvements can be made to Theorems $14.2,14.3$, and 14.4, but we shall not pursue this here. Some results on $\Delta(r, s)$ for $r, s \in \mathcal{A}(M, T)$ are given in $[\mathrm{H}]$, and for $r \in \mathcal{D}(M, T), s \in \mathcal{A}(M, T)$ in [HM].

Note that the examples listed in Theorems 1.1 and 14.2 with $\Delta(r, s)>5$ all have $\partial M=T$. Hence, combining Theorems 1.1, 14.1, 14.2, 14.3, and 14.4, we have the following.

Corollary 14.5. Suppose that $\partial M \neq T$. If $r, s \in \mathcal{B}_{+}(M, T)$, then either $\Delta(r, s) \leq$ 5 , or $M$ contains an essential annulus with exactly one boundary component on $T$.

We now give the proofs of Theorems 1.2, 1.3, and 1.4 stated in the Introduction.

Proof of Theorem 1.2. Suppose that $M \in \mathcal{A}$, but $M(r) \notin \mathcal{A}$. Then $M(r)$ may contain an essential sphere, in which case it is easy to show that $r \in \mathcal{S}(M, T)$. If $M(r)$ is irreducible then it must contain an essential torus, from which it easily 
follows (using the fact that $M(r)$ is irreducible, however) that $r \in \mathcal{T}(M, T)$. Thus if $M(r), M(s) \notin \mathcal{A}$, we must have $r, s \in \mathcal{S}(M, T) \cup \mathcal{T}(M, T)$. The result is now a consequence of Theorems 1.1, 14.1, and 14.2.

Proof of Theorem 1.3. Suppose that $M \in \mathcal{H}_{0}$, where $\partial M \neq T$, and $M(r) \notin \mathcal{H}_{0}$. Since $\partial M(r) \neq \emptyset$, it follows from [T] that either $M(r) \notin \mathcal{A}$ (in which case $r \in$ $\boldsymbol{S}(M, T) \cup \mathcal{T}(M, T))$, or $M(r)$ is Seifert fibred.

Suppose that $M(r)$ is Seifert fibred. Then $M(r)$ is irreducible and contains either an essential disk (and is homeomorphic to $S^{1} \times D^{2}$ ) or an essential annulus. Therefore $r \in \mathcal{D}(M, T) \cup \mathcal{A}(M, T)$. Also, since here $\partial M(r)$ and hence $\partial M$ consists of tori, if $M$ contained an essential annulus it would either contain an essential torus or be Seifert fibred, contradicting our hypothesis that $M \in \mathcal{H}_{0}$. Therefore $M(r)$ Seifert fibred implies that $r \in \mathcal{D}(M, T) \cup \mathcal{A}(M, T)$ and that $M$ does not contain an essential annulus.

The result now follows directly from Corollary 14.5.

Proof of Theorem 1.4. Let $M_{0}$ be the component of $M$ cut along $S$ that contains $T=\partial M$. Note that $M_{0}$ is irreducible. Consider the pair $\left(M_{0}, T\right)$, and let $r$ be a slope on $T$. Since $M \in \mathcal{A}$, standard arguments show that if $r \notin \mathcal{B}_{+}\left(M_{0}, T\right)$ then $S$ is incompressible in $M(r)$ and $M(r) \in \mathcal{A}$. It then follows from [T] that $M(r)$ is either hyperbolic or Seifert fibred.

Suppose that $M(r)$ is Seifert fibred. Then $S$ is an incompressible horizontal surface in $M(r)$, so $M(r)$ cut along $S$, that is, $M_{0}(r)$, is homeomorphic to $S \times I$. Choose a set of simple closed curves in $S$ such that $S$ cut along these curves is a disk, and consider the corresponding annuli in $S \times I$. By considering the intersection of the solid torus $V$ in $M_{0}(r)=M_{0} \cup V$ with these annuli, standard arguments show that $r \in \mathcal{A}\left(M_{0}, T\right)$.

Hence $r \notin \mathcal{B}_{+}\left(M_{0}, T\right)$ implies $M(r) \in \mathcal{H}$. The result now follows from Corollary 14.5.

\section{REFERENCES}

[BZ1] S. Boyer and X. Zhang, The semi-norm and Dehn filling, preprint.

[BZ2] S. Boyer and X. Zhang, Reducing Dehn filling and toroidal Dehn filling, Topology Appl. 68 (1996), 285-303. CMP 1996:9

[BFLW] A.M. Brunner, M.L. Frame, Y.W. Lee, and N.J. Wielenberg, Classifying torsion-free subgroups of the Picard group, Trans. Amer. Math. Soc. 282 (1984), 205-235. MR 85h:57012

[CGLS] M. Culler, C.McA. Gordon, J. Luecke, and P.B. Shalen, Dehn surgery on knots, Ann. of Math. 125 (1987), 237-300. MR 88a:57026

[GLi] C.McA. Gordon and R.A. Litherland, Incompressible planar surfaces in 3-manifolds, Topology and its Appl. 18 (1984), 121-144. MR 86e:57013

[GLu] C.McA. Gordon and J. Luecke, Reducible manifolds and Dehn surgery, Topology 35 (1996), 385-409. MR 97b:57013

[H] C. Hayashi, Dehn surgery and essential annuli, Math. Proc. Cambridge Philos. Soc. 120 (1996), 127-146. MR 97b:57014

[HM] C. Hayashi and K. Motegi, Dehn surgery on knots in solid tori creating essential annuli, Trans. Amer. Math. Soc. (to appear).

[Ho] C. Hodgson, private communication.

[L] R.A. Litherland, Surgery on knots in solid tori, II, J. London Math. Soc. 22 (2) (1980), 559-569. MR 82d:57002

[O] S. Oh, Reducible and toroidal 3-manifolds obtained by Dehn filling, Topology and its Applications (to appear). 
[T] W. Thurston, Three dimensional manifolds, Kleinian groups and hyperbolic geometry, Bull. Amer. Math. Soc. (N.S.) 6 (1982), 357-381. MR 83h:57019

[We] J.R. Weeks, Hyperbolic structures on three-manifolds, Ph.D. thesis, Princeton University, 1985.

[Wu1] Y.-Q. Wu, Incompressibility of surfaces in surgered 3-manifolds, Topology 31 (1992), 271-279. MR 94e:57027

[Wu2] Y.-Q. Wu, Dehn fillings producing reducible manifold and toroidal manifold, preprint.

Department of Mathematics, The University of Texas at Austin, Austin, Texas 78712

E-mail address: gordon@math.utexas.edu 